\title{
A Classification of some Quadratic Algebras
}

\author{
Heather C. McGilvray
}

Dissertation submitted to the Faculty of the Virginia Polytechnic Institute and State University in partial fulfillment of the requirements for the degree of

\author{
Doctor of Philosophy \\ in \\ Mathematics \\ Edward Green, Chair \\ Joseph Ball \\ Peter Linnell \\ Robert McCoy \\ Robert Olin
}

July 16, 1997

Blacksburg, Virginia

Keywords: Koszul algebra, quadratic algebra, monomial generators Copyright 1998, Heather C. McGilvray 


\section{A Classification of some Quadratic Algebras \\ Heather C. McGilvray}

\section{(ABSTRACT)}

In this paper, for a select group of quadratic algebras, we investigate restrictions necessary on the generators of the ideal for the resulting algebra to be Koszul. Techniques include the use of Gröbner bases and development of Koszul resolutions. When the quadratic algebra is Koszul, we provide the associated linear resolution of the field. When not Koszul, we describe the maps of the resolution up to the instance of nonlinearity. 


\section{Dedication}

I wish to dedicate this work to my family: my father, Ed Davis, for his understanding and sound council; my mother, Bertha Davis, for her boundless strength and love; and my husband, Jim Dobberfuhl, for the joy and laughter he brings to my life. 


\section{Acknowledgments}

I wish to thank my advisor, Dr. Green, for his guidance and support, as well as for directing me into such a demanding and wildly rewarding field of mathematics. Through him, I have gained a greater understanding of mathematics than I had dared to hope. 


\section{Contents}

1 Introduction and Background 1

2 Results $\quad 4$

3 Koszul Algebras - Part I $\quad 6$

4 Koszul Algebras - Part II $\quad 10$

5 A Non-Koszul Algebra $\quad 35$

6 Additional Classes of Koszul Algebras $\quad 57$

7 Matrix Diagrams $\quad 59$ 


\section{List of Matrix Diagrams}

Diagram 3.1 Maps of the linear resolution of the field for Pages 59-60 $I=\left\langle m_{1}, \ldots, m_{n}, X_{\gamma} X_{j_{i}}+c X_{\beta} X_{j_{i}}\right\rangle$

Diagram 3.2 Maps of the linear resolution of the field for Page 61 $I=\left\langle m_{1}, \ldots, m_{n}, X_{j_{i}} X_{\gamma}+c X_{j_{i}} X_{\beta}\right\rangle$

Diagram 3.3 Maps of the linear resolution of the field for Page 62 $I=\left\langle m_{1}, \ldots, m_{n}, X_{\beta} X_{\gamma}+c X_{\alpha} X_{\theta}\right\rangle$

Diagram 4.1 Maps of the linear resolution of the field for Page 63 $I=\left\langle m_{1}, \ldots, m_{n}, X_{j_{i}} X_{j_{k}}+c X_{j_{i}} X_{j_{l}}\right\rangle$

Diagram 4.2 Maps of the linear resolution of the field for Page 64 $I=\left\langle m_{1}, \ldots, m_{n}, X_{j_{k}} X_{j_{i}}+c X_{j_{l}} X_{j_{i}}\right\rangle$

Diagram 4.3 Maps of the linear resolution of the field for Pages $65-66$ $I=\left\langle m_{1}, \ldots, m_{n}, \sum_{i=1}^{s_{1}} c_{1}^{i} p_{1}^{i}, \ldots, \sum_{i=1}^{s_{z}} c_{z}^{i} p_{z}^{i}\right\rangle$

Diagram 5.1 Maps of the linear resolution of the field for Pages $67-72$ $I=\left\langle m_{1}, \ldots, m_{n}, X_{j_{i}} X_{\beta}+c X_{\xi} X_{j_{i}}\right\rangle$ 


\section{Chapter 1}

\section{Introduction and Background}

This paper aims to further understand for which quadratic generators of an ideal the resulting algebra will be Koszul. Additionally, we are interested in developing the associated Koszul resolutions for the algebras which are Koszul, as well as the nonlinear resolutions for the cases when the algebra is not Koszul. We begin with some fundamental definitions and concepts.

Given an ideal, $I$, and a free algebra, $K\left\langle X_{1}, \ldots, X_{t}\right\rangle$, we say the quotient

$$
\Lambda=K\left\langle X_{1}, \ldots, X_{t}\right\rangle / I
$$

is a quadratic algebra if $I$ is generated by relations $\rho=\sum_{i=1}^{n} c_{i} y_{i} z_{i}$ where $y_{i}, z_{i} \in\left\{X_{1}, \ldots, X_{t}\right\}$ and $c_{i} \in K$ for all $\mathrm{i}$. That is, $I$ is generated by linear combinations of quadratic monomials. Some quadratic algebras are additionally Koszul algebras. A quadratic algebra, $\Lambda$, is Koszul if a linear resolution of the field can be obtained [8]. We say a projective resolution of a graded $\Lambda$-module, $M, \cdots \rightarrow P_{n} \stackrel{\varphi_{n}}{\rightarrow} P_{n-1} \stackrel{\varphi_{n}-1}{\rightarrow} \cdots \stackrel{\varphi_{2}}{\rightarrow} P_{1} \stackrel{\varphi_{7}}{\rightarrow} P_{0} \stackrel{\varphi_{0}}{\rightarrow} M \rightarrow 0$ is linear if each $P_{n}$ is generated in degree $n$ and $\operatorname{ker} \varphi_{n}$ is generated in degree $n+1$ for all $n \geq 0$.

For this specific class of algebras, a projective resolution of the field, $K$, which will be of the form $\ldots \stackrel{\varphi_{3}}{\rightarrow} \Lambda^{n_{2}} \stackrel{\varphi_{2}}{\rightarrow} \Lambda^{n_{1}} \stackrel{\varphi_{3}}{\rightarrow} \Lambda^{n_{0}} \stackrel{\varphi_{0}}{\rightarrow} K \rightarrow 0$, is said to be linear provided that $\varphi_{i}$, when regarded as an $\left(n_{i-1} \times n_{i}\right)$ matrix acting on an $n_{i}$-tuple, has linear entries, for all $i$. If such a resolution exists, then $\Lambda$ will be Koszul. We note here that the above characterization of Koszul is not the classic definition as introduced by Priddy [12]. For a review of basic properties of Koszul algebras and modules, including alternate definitions of Koszul, see Backelin and Fröberg [1] and Green and Martinez-Villa [8].

Koszul algebras naturally arise in areas of mathematics such as algebraic topology, Lie theory, quantum groups, and algebraic geometry [2] [3] [4] [9] [11]. Considering the importance of these algebras in such varied topics, it is desirable to definitively isolate necessary and sufficient conditions on the ideals for the resulting quadratic algebra to be Koszul. However, in practice, the resolutions obtained are difficult to classify and describe. As a result, not much is known about what makes an algebra Koszul. 
We do know that if the ideal, which is generated by linear combinations of quadratic monomials, further has a quadratic (noncommutative) Gröbner basis, then the resulting quadratic algebra will be Koszul. This is not a necessary condition, however. A Gröbner basis is a generating set of relations that also satisfies further criterion. In order to understand the additional conditions for a generating set to be a Gröbner basis, we must first impose an order on the monomials of the free algebra. More specifically, we need an admissible ordering. We now give some common examples of admissible orders.

(left)length-lexicographic order For length lexicographic order, we arbitrarily order the variables of the free algebra: $X_{1}<X_{2}<\cdots<X_{t}$. Then, if $M=X_{j_{1}} X_{j_{2}} \cdots X_{j_{m}}$ and $N=X_{k_{1}} X_{k_{2}} \cdots X_{k_{n}}$ are monomials of the free algebra, $M<N$ if $m<n$. If $m=n$, then $M<N$ if for some $1 \leq i \leq m, X_{j_{l}}=X_{k_{l}}$ for all $l<i$ and $X_{j_{i}}<X_{k_{i}}$. [7]

(left)weight-lexicographic order For weight lexicographic order, we start with a set map $w:\left\{X_{1}, \ldots, X_{t}\right\} \longrightarrow\{1,2, \ldots\}$. We extend to $w:\{$ monomials $\} \longrightarrow\{1,2, \ldots\}$ by $w\left(X_{j_{1}} X_{j_{2}} \cdots X_{j_{m}}\right)=\sum_{i=1}^{m} w\left(X_{j_{i}}\right)$. Then, the variables are ordered by $X_{i}<X_{j}$ if $w\left(X_{i}\right)<w\left(X_{j}\right)$. Finally, if $M=X_{j_{1}} X_{j_{2}} \cdots X_{j_{m}}$ and $N=X_{k_{1}} X_{k_{2}} \cdots X_{k_{n}}$ are monomials of the free algebra, $M<N$ if $w(M)<w(N)$. If $w(M)=w(N)$, then $M<N$ if $M<N$ under length lexicographical order. [7]

Once an admissible order is choosen, for any polynomial in the noncommutative polynomial ring in $t$-variables, we can identify the leading term of the polynomial; this term is also known as the "tip" of the polynomial. A generating set, $\mathcal{G}$, of an ideal, $I$, is a noncommutative Gröbner basis for $I$ provided that the ideal generated by the set of leading terms of $\mathcal{G}$ is the same ideal generated by the set of leading terms of $I$;

$$
\text { i.e. }\langle\operatorname{Tip}(\mathcal{G})\rangle=\langle\operatorname{Tip}(I)\rangle
$$

This means that if $p$ is a polynomial in the ideal, then there is an element of the Gröbner basis whose leading term divides the leading term of $p$.

Not every generating set for an ideal is also a Gröbner basis for the ideal. However, a Gröbner basis may be obtained from any generating set via the Buchberger Algorithm [5], in the commutative case, and the Buchberger-Mora-Farkas-Green Algorithm [7] in the noncommutative case. In the commutative case, the algorithm always terminates in a finite number of steps, resulting in a finite Gröbner basis. However, the noncommutative algorithm does not always terminate or result in a finite Gröbner basis. Hence, in the noncommutative case, while the Gröbner basis of an ideal always exists, it is not necessarily finite.

So, if, from the set of quadratic relations of the ideal, we obtain a (finite) quadratic Gröbner basis, then the algebra is Koszul [9]. We wish to emphasize again that this is not a necessary condition. That is, conversely, if, using the Buchberger-Mora-Farkas-Green Algorithm, we obtain a nonquadratic term to add to the Gröbner basis, then there is inconclusive evidence as to the Koszul nature of the algebra. In this case, we must employ some other method of determining if the algebra is Koszul. 
It has been shown by E. Green and D. Zacharia [10] that if the ideal is generated by quadratic monomials, then it will have a quadratic Gröbner basis, and hence, the resulting algebra will be Koszul [8]. Thus, it is certainly true that an algebra which is the quotient of an ideal generated by perfect square monomials is Koszul. We wish to understand the situation arising when, to the above ideal, we add a nonmonomial generator. This creates a class of algebras of the form

$$
K\left\langle X_{1}, \cdots, X_{t}\right\rangle /\left\langle m_{1}, \cdots, m_{n}, p+c q\right\rangle
$$

where $m_{i}=X_{j_{i}}^{2}$ is a perfect square for all $i, p$ and $q$ are monomials and $c \in K$. For this class, the Gröbner basis of the ideal is not always quadratic. It is this class of algebras, the Gröbner bases of the ideals, and the projective resolutions of the field that we explore in this paper. Additionally, we investigate the class of the form

$$
K\left\langle X_{1}, \cdots, X_{t}\right\rangle /\left\langle m_{1}, \cdots, m_{n}, \sum c_{1}^{i} p_{1}^{i}, \cdots, \sum c_{z}^{i} p_{z}^{i}\right\rangle
$$

where $c_{j}^{i} \in K, m_{i}, p_{j}^{i}$ are perfect squares for all $i, j$.

Lastly, once the prior algebras are explored, we then apply dual basis theory to the Koszul algebras, expanding the known classes of quadratic Koszul algebras. 


\section{Chapter 2}

\section{Results}

We began this work with the knowledge that an algebra of the form

$$
K\left\langle X_{1}, \cdots, X_{t}\right\rangle /\left\langle m_{1}, \cdots, m_{n}\right\rangle
$$

where each $m_{i}=X_{j_{i}}^{2}$ is a perfect square, will be Koszul since the Gröbner basis of the ideal is always quadratic. We found that adding a single nonmonomial term to the generating set of the previous ideal produces ideals and algebras of varied properties. Of the investigated algebras of the form

$$
K\left\langle X_{1}, \cdots, X_{t}\right\rangle /\left\langle m_{1}, \cdots, m_{n}, p+c q\right\rangle
$$

where $m_{i}=X_{j_{i}}^{2}$ is a perfect square for all $i, p$ and $q$ are monomials and $c \in K$, not all of the Gröbner bases of the ideals are quadratic, nor are all of the resulting algebras Koszul. However, those algebras that are Koszul, regardless of the status of their Gröbner bases, can be divided into two distinct, but similar, classes. The first can be characterized by having resolutions of the form :

$$
\ldots \stackrel{\varphi}{\rightarrow} \Lambda^{n+1} \stackrel{\varphi}{\rightarrow} \Lambda^{n+1} \stackrel{\varphi_{2}}{\rightarrow} \Lambda^{t}\left(X_{1} \stackrel{\cdots}{\rightarrow} X_{t}\right) \Lambda \rightarrow K \rightarrow 0
$$

This includes the algebras

$$
\begin{aligned}
\Lambda & =K\left\langle X_{1}, \cdots, X_{t}\right\rangle /\left\langle m_{1}, \cdots, m_{n}, X_{\gamma} X_{j_{i}}+c X_{\beta} X_{j_{i}}\right\rangle \\
\Lambda & =K\left\langle X_{1}, \cdots, X_{t}\right\rangle /\left\langle m_{1}, \cdots, m_{n}, X_{j_{i}} X_{\gamma}+c X_{j_{i}} X_{\beta}\right\rangle \\
\Lambda & =K\left\langle X_{1}, \cdots, X_{t}\right\rangle /\left\langle m_{1}, \cdots, m_{n}, X_{j_{i}} X_{j_{k}}+c X_{j_{i}} X_{j_{l}}\right\rangle
\end{aligned}
$$

and

$$
\Lambda=K\left\langle X_{1}, \cdots, X_{t}\right\rangle /\left\langle m_{1}, \cdots, m_{n}, X_{j_{k}} X_{j_{i}}+c X_{j_{l}} X_{j_{i}}\right\rangle
$$

where $\gamma \neq \beta, j_{v}$ for all $v$ and $i, k, l$ are all distinct, as described in Theorems 3.1, 3.2, 4.1, and 4.2. Each resolution consists of the same modules, and, while the algebras produce 
different maps, in each case the maps and modules begin to repeat at the first appearance of the $\Lambda$-module $\Lambda^{n+1}$.

Additionally, if we look at the maps associated with the algebras

$$
\Lambda=K\left\langle X_{1}, \cdots, X_{t}\right\rangle /\left\langle m_{1}, \cdots, m_{n}, X_{\gamma} X_{j_{i}}+c X_{\beta} X_{j_{i}}\right\rangle
$$

and

$$
\Lambda=K\left\langle X_{1}, \cdots, X_{t}\right\rangle /\left\langle m_{1}, \cdots, m_{n}, X_{j_{k}} X_{j_{i}}+c X_{j_{l}} X_{j_{i}}\right\rangle
$$

where $\gamma \neq \beta, j_{v}$ for all $v$ and $i, k, l$ all distinct, as described in Theorems 3.1 and 4.2, only one of which has a quadratic Gröbner basis, we find they are remarkably similar. They differ only in the placement of a single column in each matrix. However, of even more interest is the identical nature of the maps associated with the algebras

$$
\Lambda=K\left\langle X_{1}, \cdots, X_{t}\right\rangle /\left\langle m_{1}, \cdots, m_{n}, X_{j_{i}} X_{\gamma}+c X_{j_{i}} X_{\beta}\right\rangle
$$

and

$$
\Lambda=K\left\langle X_{1}, \cdots, X_{t}\right\rangle /\left\langle m_{1}, \cdots, m_{n}, X_{j_{i}} X_{j_{k}}+c X_{j_{i}} X_{j_{l}}\right\rangle
$$

where $\gamma \neq \beta, j_{v}$ for all $v$ and $i, k, l$ are all distinct, from Theorems 3.2 and 4.1. Again, only one of which has a quadratic Gröbner basis.

The second class consists of the algebras

$$
\Lambda=K\left\langle X_{1}, \cdots, X_{t}\right\rangle /\left\langle m_{1}, \cdots, m_{n}, X_{\beta} X_{\gamma}+c X_{\alpha} X_{\theta}\right\rangle
$$

and

$$
\Lambda=K\left\langle X_{1}, \cdots, X_{t}\right\rangle /\left\langle m_{1}, \cdots, m_{n}, X_{s}^{2}+c X_{u}^{2}\right\rangle
$$

where $\beta, \gamma \neq j_{v}$ for all $v, \beta \neq \gamma$ and $s \neq u$, as described in Theorems 3.3 and 4.3. We note that 2.1 is obtained by reducing the algebra described in Theorem 4.3 down to the simplest nontrivial case. For this class, we again have for both algebras resolutions of the form:

$$
\ldots \stackrel{\varphi}{\rightarrow} \Lambda^{n} \stackrel{\varphi}{\rightarrow} \Lambda^{n} \stackrel{\varphi_{3}}{\rightarrow} \Lambda^{n+1} \stackrel{\varphi_{2}}{\rightarrow} \Lambda^{t}\left(X_{1} \stackrel{\cdots}{\rightarrow} X_{t}\right) \Lambda \rightarrow K \rightarrow 0
$$

with repetition now occuring at the first appearance of the $\Lambda$-module $\Lambda^{n}$. In fact, for both algebras the resolution is made up of the same modules, and, as above in the case of the common term on the left, maps of identical form.

For the class of algebras found not to be Koszul, namely

$$
\Lambda=K\left\langle X_{1}, \cdots, X_{t}\right\rangle /\left\langle m_{1}, \cdots, m_{n}, X_{j_{i}} X_{\beta}+c X_{\xi} X_{j_{i}}\right\rangle
$$

where $\beta \neq \xi$ and $\beta, \xi \neq j_{i}$, we found that the resolution of the field bore a marked resemblence to the first class of Koszul algebras above, up to the first appearance of the $\Lambda$-module $\Lambda^{n+1}$. The difference is that instead of repetition beginning at this location in the resolution, as occurs for the first type of Koszul algebras, we get the first nonlinear map. In both cases, however, it is at the first appearance of the $\Lambda$-module, $\Lambda^{n+1}$, that something of import occurs. 


\section{Chapter 3}

\section{Koszul Algebras - Part I}

Throughout this chapter, $I=\left\langle m_{1}, \ldots, m_{n}, p+c q\right\rangle$ for $m_{1}=X_{j_{1}}^{2}, \ldots, m_{n}=X_{j_{n}}^{2}$ perfect square monomials and $p$ and $q$ monomials in $K\left\langle X_{1}, \ldots, X_{t}\right\rangle, c \in K$. In addition, $\Lambda=$ $K\left\langle X_{1}, \ldots, X_{t}\right\rangle /\left\langle m_{1}, \ldots, m_{n}, p+c q\right\rangle$.

Lemma 3.1 If $p+c q=\left\{\begin{array}{l}X_{j_{i}} X_{\gamma}+c X_{j_{i}} X_{\beta} \\ X_{\gamma} X_{j_{i}}+c X_{\beta} X_{j_{i}}\end{array}\right.$, where $\gamma \neq \beta, j_{k}$ for all $k$, then I has a quadratic Gröbner basis.

Proof WLOG, let $p+c q=X_{j_{i}} X_{\gamma}+c X_{j_{i}} X_{\beta}$, where $X_{\gamma}, X_{\beta} \in\left\{X_{1}, \ldots, X_{t}\right\}$. Then, $X_{\gamma}, X_{\beta} \neq X_{j_{i}}$ and $X_{\gamma} \neq X_{j_{k}}$ for all k. Choose an order so that $\operatorname{tip}(p+c q)=X_{j_{i}} X_{\gamma}$. Since $X_{\gamma} \neq X_{j_{i}}, p+c q$ has no self overlap. Hence, the only overlap involving $p+c q$ occurs with $m_{i}$ :

$$
o\left(p+c q, m_{i}\right)=X_{j_{i}}\left(X_{j_{i}} X_{\gamma}+c X_{j_{i}} X_{\beta}\right)-\left(X_{j_{i}}^{2}\right) X_{\gamma}=c X_{j_{i}}^{2} X_{\beta} \stackrel{m_{i}}{\longmapsto} 0
$$

Thus, $\left\{m_{1}, \ldots, m_{n}, p+c q\right\}$ is a quadratic Gröbner basis for I.

Rephrasing, if $\operatorname{supp}(\mathrm{p}) \cap \operatorname{supp}(\mathrm{q}) \cap \operatorname{supp}\left(m_{i}\right) \neq \phi$, for some $i, X_{l}^{2}$ does not divide $p q, q p \forall l$ and $\operatorname{supp}(\mathrm{p}) \cap \operatorname{supp}\left(m_{j}\right)=\phi$ for all $j \neq i$, then $I$ has a quadratic Gröbner basis. We remark that if $\beta=\gamma$ in the above description of $p+c q$, then $I$ is generated by quadratic monomials and hence has a quadratic Gröbner basis by remarks in Chapter 1.

Corollary 3.1 $\Lambda=K\left\langle X_{1}, \ldots, X_{t}\right\rangle /\left\langle m_{1}, \ldots, m_{n}, p+c q\right\rangle$, for $p+c q$ as defined in Lemma 3.1, is Koszul.

Lemma 3.2 If $p+c q=X_{j_{i}} X_{\beta}+c X_{\beta} X_{j_{i}}$ with $\beta \neq j_{i}$ and $c \in K$, then

$$
I=\left\langle m_{1}, \ldots, m_{n}, p+c q\right\rangle
$$


has a quadratic Gröbner basis.

Proof Choose an order so that tip $(p+c q)=X_{j_{i}} X_{\beta}=p$. Since $\mathrm{p}$ is not a perfect square, $p+c q$ has no selfoverlap. And,

$$
\begin{gathered}
o\left(p+c q, m_{i}\right)=X_{j_{i}}\left(X_{j_{i}} X_{\beta}+c X_{\beta} X_{j_{i}}\right)-\left(X_{j_{i}}^{2}\right) X_{\beta}=c X_{j_{i}} X_{\beta} X_{j_{i}} \\
c X_{j_{i}} X_{\beta} X_{j_{i}}-c\left(X_{j_{i}} X_{\beta}+c X_{\beta} X_{j_{i}}\right) X_{j_{i}}=-c^{2} X_{\beta} X_{j_{i}}^{2} \stackrel{m_{i}}{\longmapsto} 0
\end{gathered}
$$

Now, suppose $X_{\beta} \mid m_{k}$ for some k (i.e., $X_{\beta}=X_{j_{k}}$ ). Then,

$$
\begin{gathered}
o\left(p+c q, m_{k}\right)=\left(X_{j_{i}} X_{\beta}+c X_{\beta} X_{j_{i}}\right) X_{j_{k}}-X_{j_{i}} X_{j_{k}}^{2}=c X_{\beta} X_{j_{i}} X_{\beta} \\
c X_{\beta} X_{j_{i}} X_{\beta}-c X_{\beta}\left(X_{j_{i}} X_{\beta}+c X_{\beta} X_{j_{i}}\right)=-c^{2} X_{\beta}^{2} X_{j_{i}} \stackrel{m_{k}}{\longmapsto} 0
\end{gathered}
$$

Thus, $\left\{m_{1}, \ldots, m_{n}, p+c q\right\}$ is a quadratic Gröbner basis for I.

That is, if neither $p$ nor $q$ is a perfect square, $\operatorname{supp}(p)=\operatorname{supp}(q), \operatorname{supp}(p) \cap \operatorname{supp}\left(m_{i}\right) \neq \phi$ for some i, and $p \neq q$, then $I$ has a quadratic Gröbner basis.

Corollary 3.2 $\Lambda=K\left\langle X_{1}, \ldots, X_{t}\right\rangle /\left\langle m_{1}, \ldots, m_{n}, X_{j_{i}} X_{\beta}+c X_{\beta} X_{j_{i}}\right\rangle$ is Koszul.

Lemma 3.3 If $p+c q=X_{\beta} X_{\gamma}+X_{\alpha} X_{\theta}$, for $\beta, \gamma \neq j_{l}$ for all $l$ and $\beta \neq \gamma$, then $I=$ $\left\langle m_{1}, \ldots, m_{n}, p+c q\right\rangle$ has a quadratic Gröbner basis.

Proof Choose an order so that $\operatorname{tip}(p+c q)=X_{\beta} X_{\gamma}$. Now, since $\beta, \gamma \neq j_{l}$ for all $l, p+c q$ has no overlaps with the $m_{i}$ 's. And, since $\mathrm{p}$ is not a perfect square, $p+c q$ has no selfoverlaps. Thus, $\left\{m_{1}, \ldots, m_{n}, p+c q\right\}$ is a quadratic Gröbner basis for I.

That is, if $\mathrm{p}$ is not a perfect square and $\operatorname{supp}(\mathrm{p}) \cap \operatorname{supp}\left(m_{i}\right)=\phi$ for all $\mathrm{i}$, then $I$ has a quadratic Gröbner basis.

Corollary 3.3 $\Lambda=K\left\langle X_{1}, \ldots, X_{t}\right\rangle /\left\langle m_{1}, \ldots, m_{n}, p+c q\right\rangle$, for $p+c q$ as defined in Lemma 3.3, is Koszul.

Since the algebras in Corollaries 3.1 and 3.3 are Koszul, we know that there exist linear resolutions of the field, $k$, for each algebra. Showing the ideal has a quadratic Gröbner basis, while one of the simplest ways of proving Koszul, lacks the ability to illuminate the underlying structure of the algebra. Addressing this, we now give descriptions, without proofs, of the matrices associated with the linear resolutions of the field. 
Theorem 3.1 For

$$
\Lambda=K\left\langle X_{1}, \ldots, X_{t}\right\rangle /\left\langle m_{1}, \ldots, m_{n}, X_{\gamma} X_{j_{i}}+c X_{\beta} X_{j_{i}}\right\rangle
$$

where $X_{j_{1}}<\cdots<X_{j_{k}}<X_{\beta}<X_{j_{k+1}}<\cdots<X_{j_{l}}<X_{\gamma}<X_{j_{l+1}}<\cdots<X_{j_{n}}$, we have the following linear resolution of the field:

$$
\cdots \stackrel{\varphi}{\longrightarrow} \Lambda^{n+1} \stackrel{\varphi}{\longrightarrow} \Lambda^{n+1} \stackrel{\varphi 2}{\longrightarrow} \Lambda^{t} \stackrel{\left(X_{1} \stackrel{\cdots}{\longrightarrow} X_{t}\right)}{\longrightarrow} \Lambda \longrightarrow k \longrightarrow 0
$$

where $\varphi_{2}=\left(a_{r s}\right)$ and $\varphi=\left(b_{r s}\right)$ for

$$
a_{r s}= \begin{cases}X_{r} & \text { if } r=j_{s} \text { and } 1 \leq s \leq l \\ X_{r} & \text { if } r=j_{s-1} \text { and } l+2 \leq s \leq n+1 \\ c X_{j_{i}} & \text { if } r=\beta \text { and } s=l+1 \\ X_{j_{i}} & \text { if } r=\gamma \text { and } s=l+1 \\ 0 & \text { if otherwise }\end{cases}
$$

(See Diagram 3.1)

$$
b_{r s}= \begin{cases}X_{j_{r}} & \text { if } r=s \text { and } 1 \leq r \leq l \\ X_{j_{i}} & \text { if } r=s=l+1 \\ X_{j_{r-1}} & \text { if } r=s \text { and } l+2 \leq r \leq n+1 \\ 0 & \text { if otherwise }\end{cases}
$$

(See Diagram 3.1)

Theorem 3.2 For

$$
\Lambda=K\left\langle X_{1}, \ldots, X_{t}\right\rangle /\left\langle m_{1}, \ldots, m_{n}, X_{j_{i}} X_{\gamma}+c X_{j_{i}} X_{\beta}\right\rangle
$$

we have the following linear resolution of the field:

$$
\ldots \stackrel{\varphi}{\longrightarrow} \Lambda^{n+1} \stackrel{\varphi}{\longrightarrow} \Lambda^{n+1} \stackrel{\varphi_{2}}{\longrightarrow} \Lambda^{t}\left(X_{1} \stackrel{\cdots}{\longrightarrow} X_{t}\right) \Lambda \longrightarrow k \longrightarrow 0
$$

where $\varphi_{2}=\left(a_{r s}\right)$ and $\varphi=\left(b_{r s}\right)$ for

$$
a_{r s}= \begin{cases}X_{r} & \text { if } 1 \leq s \leq i \text { and } r=j_{s} \\ X_{\gamma}+c X_{\beta} & \text { if } s=i+1 \text { and } r=j_{i} \\ X_{r} & \text { if } i+2 \leq s \leq n+1 \text { and } r=j_{s-1} \\ 0 & \text { if otherwise }\end{cases}
$$

(See Diagram 3.2) 
H. C. McGilvray

$$
b_{r s}= \begin{cases}X_{j_{r}} & \text { if } r=s \text { and } 1 \leq r \leq i \\ X_{\gamma}+c X_{\beta} & \text { if } r=i \text { and } s=i+1 \\ X_{j_{r-1}} & \text { if } r=s \text { and } i+2 \leq r \leq n+1 \\ 0 & \text { if otherwise }\end{cases}
$$

(See Diagram 3.2)

Theorem 3.3 For

$$
\Lambda=K\left\langle X_{1}, \ldots, X_{t}\right\rangle /\left\langle m_{1}, \ldots, m_{n}, X_{\beta} X_{\gamma}+X_{\alpha} X_{\theta}\right\rangle
$$

where $\beta, \gamma \neq j_{l}$ for all $l, \beta \neq \gamma$ and $X_{j_{1}}<\ldots<X_{j_{k}}<X_{\beta}<X_{j_{k+1}}<\ldots<X_{j_{n}}$, we have the following linear resolution of the field:

$$
\cdots \stackrel{\varphi}{\longrightarrow} \Lambda^{n} \stackrel{\varphi}{\longrightarrow} \Lambda^{n} \stackrel{\varphi_{3}}{\longrightarrow} \Lambda^{n+1} \stackrel{\varphi_{2}}{\longrightarrow} \Lambda^{t}\left(X_{1} \stackrel{\cdots}{\longrightarrow} X_{t}\right) \Lambda \longrightarrow k \longrightarrow 0
$$

where $\varphi_{2}=\left(a_{r s}\right), \varphi_{3}=\left(b_{r s}\right)$ and $\varphi=\left(c_{r s}\right)$ for

$$
a_{r s}= \begin{cases}X_{r} & \text { if } 1 \leq s \leq k \text { and } r=j_{s} \\ X_{\theta} & \text { if } r=\alpha \text { and } s=k+1 \\ X_{\gamma} & \text { if } s=k+1 \text { and } r=\beta \\ X_{r} & \text { if } k+2 \leq s \leq n+1 \text { and } r=j_{s-1} \\ 0 & \text { if otherwise }\end{cases}
$$

(See Diagram 3.3)

$$
b_{r s}= \begin{cases}X_{j_{r}} & \text { if } r=s \text { and } 1 \leq r \leq k \\ X_{j_{r-1}} & \text { if } r=s+1 \text { and } k+2 \leq r \leq n+1 \\ 0 & \text { if otherwise }\end{cases}
$$

(See Diagram 3.3)

$$
c_{r s}= \begin{cases}X_{j_{r}} & \text { if } r=s \\ 0 & \text { if otherwise }\end{cases}
$$

(See Diagram 3.3) 


\section{Chapter 4}

\section{Koszul Algebras - Part II}

In this chapter, for some results, we expand the generators of the ideal from those of Chapter 3. We will state when results are for the expanded generating set. However, throughout, $m_{1}=X_{j_{1}}^{2}, \ldots, m_{n}=X_{j_{n}}^{2}$ are perfect square monomials in $K\left\langle X_{1}, \ldots, X_{t}\right\rangle$ and $\Lambda=K\left\langle X_{1}, \ldots, X_{t}\right\rangle / I$. Additionally, $\bar{\gamma}$ means $\gamma+I$.

Lemma 4.1 If $p+c q=\left\{\begin{array}{l}X_{j_{i}} X_{j_{k}}+c X_{j_{i}} X_{j_{l}} \\ X_{j_{k}} X_{j_{i}}+c X_{j_{l}} X_{j_{i}}\end{array}\right.$, with $i, k$, and $l$ distinct and $c \in K$, then $I=\left\langle m_{1}, \ldots, m_{n}, p+c q\right\rangle$ has no quadratic Gröbner basis.

Proof Let $p+c q=X_{j_{i}} X_{j_{k}}+c X_{j_{i}} X_{j_{l}}$ and $\operatorname{suppose} \operatorname{tip}(p+c q)=X_{j_{i}} X_{j_{k}}\left(X_{j_{k}} X_{j_{i}}+c X_{j_{l}} X_{j_{i}}\right.$ is analogous). Then, $p+c q$ overlaps with $m_{k}$. And,

$$
\begin{aligned}
& o\left(p+c q, m_{k}\right)= \\
& \quad\left(X_{j_{i}} X_{j_{k}}+c X_{j_{i}} X_{j_{l}}\right) X_{j_{k}}-X_{j_{i}}\left(X_{j_{k}}^{2}\right) \\
& =c X_{j_{i}} X_{j_{l}} X_{j_{k}}
\end{aligned}
$$

Now, $c X_{j_{i}} X_{j_{l}} X_{j_{k}}$ is irreducible since $i \neq j$ and $j \neq k$. Thus, we have an irreducible cubic term to add to the Gröbner basis for I.

That is, if, for some distinct $i, j$, and $k, \operatorname{supp}(p) \cap \operatorname{supp}\left(m_{k}\right) \neq \phi, \operatorname{supp}(q) \cap \operatorname{supp}\left(m_{l}\right) \neq \phi$, $\operatorname{supp}(p) \cap \operatorname{supp}(q) \cap \operatorname{supp}\left(m_{i}\right) \neq \phi$, and $X_{j}^{2}$ does not divide $p q$ or $q p$ for all $j$, then $I=$ $\left\langle m_{1}, \ldots, m_{n}, p+c q\right\rangle$ has no quadratic Gröbner basis. 
Lemma 4.2 If $p_{1}^{1}, \ldots, p_{z}^{s_{z}}$ are distinct perfect square monomials in $K\left\langle X_{1}, \ldots, X_{t}\right\rangle$ with $p_{i}^{j}=$ $X_{k_{j}^{i}}^{2} \neq m_{v}$ for all $i, j, k, v$, then,

$$
I=\left\langle m_{1}, \ldots, m_{n}, \sum_{i=1}^{s_{1}} c_{1}^{i} p_{1}^{i}, \ldots, \sum_{i=1}^{s_{z}} c_{z}^{i} p_{z}^{i}\right\rangle
$$

has no quadratic Gröbner basis.

Proof Fix i. WLOG, we may assume that $\operatorname{tip}\left(\sum c_{j}^{i} p_{j}^{i}\right)=p_{j}^{1}$.

Consider

$$
\begin{aligned}
o\left(\sum c_{1}^{i} p_{1}^{i}, \sum c_{1}^{i} p_{1}^{i}\right) & =X_{k_{1}^{1}}\left(\sum c_{1}^{i} p_{1}^{i}\right)-\left(\sum c_{1}^{i} p_{1}^{i}\right) X_{k_{1}^{1}} \\
& =\sum c_{1}^{i} X_{k_{1}^{1}} p_{1}^{i}-\sum c_{1}^{i} p_{1}^{i} X_{k_{1}^{1}} \\
& =\sum_{i=2}^{s_{1}} c_{1}^{i} X_{k_{1}^{1}} p_{1}^{i}-\sum_{i=2}^{s_{1}} c_{1}^{i} p_{1}^{i} X_{k_{1}^{1}}
\end{aligned}
$$

Now, since each $p_{1}^{i}$ is distinct, there is no cancellation between terms of the sums. Since $p_{1}^{i}$ is a perfect square for all $\mathrm{i}$, we can't reduce using $p_{1}^{1}$. Also, no $m_{i}$ can reduce the overlap as the $p_{j}^{i}$ 's are distinct from the $m_{i}$ 's. We can't reduce using another sum since each $p_{i}^{j}$ is distinct. Hence, we have irreducible cubic terms to add to the Gröbner basis for I. Thus, $I=\left\langle m_{1}, \ldots, m_{n}, \sum_{i=1}^{s_{1}} c_{1}^{i} p_{1}^{i}, \ldots, \sum_{i=1}^{s_{z}} c_{z}^{i} p_{z}^{i}\right\rangle$ has no quadratic Gröbner basis.

Thus, we see that for ideals with generators as described in Lemma 4.1 and Lemma 4.2, the resulting algebras do not have the ready implication of Koszul. However, as remarked in Chapter 1, the existence of a quadratic Gröbner basis, while a sufficient condition for the algebra to be Koszul, is not a necessary one. As we will see, the quadratic algebras generated from the ideals of Lemma 4.1 and Lemma 4.2 exemplify this fact.

For the ideal $I=\left\langle m_{1}, \ldots, m_{n}, X_{j_{i}} X_{j_{k}}+c X_{j_{i}} X_{j_{l}}\right\rangle$, we begin with a proposition clarifying ideal membership.

Proposition 4.1 Let $I=\left\langle m_{1}, \ldots, m_{n}, p+c q\right\rangle$ be an ideal in $K\left\langle X_{1}, \ldots, X_{t}\right\rangle$ where $m_{s}=X_{j_{s}}^{2}$ for all $s$ and $p+c q=X_{j_{i}} X_{j_{k}}+c X_{j_{i}} X_{j_{l}}$. If $X_{s} \gamma \in I$, then $\bar{\gamma} \equiv \overline{X_{s} \delta+\left(X_{j_{k}}+c X_{j_{l}}\right) \nu}$ for some $\delta, \nu$.

Proof Since $X_{s} \gamma \in I$, we can write

$$
X_{s} \gamma=\sum_{a=1}^{n} \beta_{a} m_{a} \lambda_{a}+\beta(p+c q) \lambda
$$


for some polynomials $\beta_{a}, \lambda_{a}, \beta, \lambda$. Rewriting,

$$
\begin{gathered}
X_{s} \gamma=\sum_{a=1}^{n_{1}} \mu_{a}^{1} m_{1} \rho_{a}^{1}+\sum_{a=1}^{n_{2}} \mu_{a}^{2} m_{2} \rho_{a}^{2}+\cdots+\sum_{a=1}^{n_{n}} \mu_{a}^{n} m_{n} \rho_{a}^{n} \\
+\sum_{a=1}^{n_{\text {sum }}} \mu_{a}(p+c q) \rho_{a}
\end{gathered}
$$

where $\mu_{a}^{j}, \rho_{a}^{j}, \mu_{a}, \rho_{a}$ are all monomials. Note that for fixed a, $X_{s}$ is a prefix of $\mu_{a}(p+c q) \rho_{a}$ even if $\mu_{a} \in K \backslash 0$. Now, since $X_{s}$ divides the lefthand side of the equation, $X_{s}$ must divide a subsum of the right hand side. That is,

$$
X_{s} \mid \sum_{\text {subsum }} \mu_{a}^{1} m_{1} \rho_{a}^{1}+\cdots+\sum_{\text {subsum }} \mu_{a}^{n} m_{n} \rho_{a}^{n}+\sum_{\text {subsum }} \mu_{a}(p+c q) \rho_{a}
$$

So,

$$
X_{s} \gamma=\underbrace{\left(\sum_{\text {subsum }} \mu_{a}^{1} m_{1} \rho_{a}^{1}+\cdots+\sum_{\text {subsum }} \mu_{a}^{n} m_{n} \rho_{a}^{n}+\sum_{\text {subsum }} \mu_{a}(p+c q) \rho_{a}\right)}_{\text {divisible by } X_{s}}+\underbrace{\Delta}_{X_{s} \nvdash \Delta}
$$

Since any term in the leftover part (in $\Delta$ ) does not begin with $X_{s}$, it must cancel with another term in $\Delta$. Hence, the leftover terms sum to zero. That is, $\Delta=0$. Thus,

$$
X_{s} \gamma=\underbrace{\sum_{\text {subsum }} \mu_{a}^{1} m_{1} \rho_{a}^{1}+\cdots+\sum_{\text {subsum }} \mu_{a}^{n} m_{n} \rho_{a}^{n}+\mu_{a}(p+c q) \rho_{a}}_{\text {all divisible by } X_{s}}
$$

First note that if $s \neq j_{v}$ for some v, then $\mu_{a}^{j}, \mu_{a} \notin K \backslash 0$ for all a and j. In this case, $\mu_{a}^{j}=X_{s} \sigma_{a}^{j}$ and $\mu_{a}=X_{s} \sigma_{a}$ for some monomials $\sigma_{a}^{j}$ and $\sigma_{a}$. So,

$$
X_{s} \gamma=\sum_{\text {subsum }} X_{s} \sigma_{a}^{1} m_{1} \rho_{a}^{1}+\cdots+\sum_{\text {subsum }} X_{s} \sigma_{a}^{n} m_{n} \rho_{a}^{n}+\sum_{\text {subsum }} X_{s} \sigma_{a}(p+c q) \rho_{a}
$$

Thus, for $\delta, \nu=0$,

$$
\bar{\gamma} \equiv \overline{X_{s} \delta+\left(X_{j_{k}}+c X_{j_{l}}\right) \nu}
$$

Now suppose $s=j_{v}$ for some $v \in\{1, \ldots, n\} \backslash\{i\}$ In this case, for all $j \neq v, \mu_{a}, \mu_{a}^{j} \notin K \backslash 0$. So, $\mu_{a}=X_{j_{v}} \sigma_{a}$ and $\mu_{a}^{j}=X_{j_{v}} \sigma_{a}^{j}$ for some monomials $\sigma_{a}$ and $\sigma_{a}^{j}$.

$$
X_{j_{v}} \gamma=\sum_{j \neq v}\left(\sum_{\text {subsum }} X_{j_{v}} \sigma_{a}^{j} m_{j} \rho_{a}^{j}\right)+\sum_{\text {subsum }} \mu_{a}^{v} m_{v} \rho_{a}^{v}+\sum_{\text {subsum }} X_{j_{v}} \sigma_{a}(p+c q) \rho_{a}
$$

If $\mu_{1}^{v}, \ldots, \mu_{\beta}^{v} \in K \backslash 0$ and $\mu_{\beta+1}^{v}$, etc $\notin K \backslash 0$, then

$$
X_{j_{v}} \gamma=\sum_{j \neq v}\left(\sum_{\text {subsum }} X_{j_{v}} \sigma_{a}^{j} m_{j} \rho_{a}^{j}\right)+\sum_{a=1}^{\beta} m_{v}\left(\mu_{a}^{v} \rho_{a}^{v}\right)+\sum_{a \geq \beta+1} X_{j_{v}} \sigma_{a}^{v} m_{v} \rho_{a}^{v}+\sum_{\text {subsum }} X_{j_{v}} \sigma_{a}(p+c q) \rho_{a}
$$


Thus, for $\delta=\sum_{a=1}^{\beta} \mu_{a}^{v} \rho_{a}^{v}$ and $\nu=0$,

$$
\bar{\gamma} \equiv \overline{X_{s} \delta+\left(X_{j_{k}}+c X_{j_{l}}\right) \nu}
$$

Lastly, if $s=j_{i}$, we again have that for $j \neq i, \mu_{a}^{j}=X_{j_{i}} \sigma_{a}^{j}$ for some monomials $\sigma_{a}^{j}$. Suppose $\mu_{1}^{i}, \ldots, \mu_{\beta}^{i}, \mu_{1}, \ldots, \mu_{\mu} \in K \backslash 0$ and $\mu_{\beta+1}^{i}$, etc., $\mu_{\mu+1}$, etc. $\notin K \backslash 0$. Then, we have,

$$
\begin{aligned}
X_{j_{i}} \gamma= & \sum_{j \neq i}\left(\sum_{\text {subsum }} X_{j_{i}} \sigma_{a}^{j} m_{j} \rho_{a}^{j}\right)+\sum_{a+1}^{\beta} m_{i}\left(\mu_{a}^{i} \rho_{a}^{i}\right)+\sum_{a \geq \beta+1} X_{j_{i}} \sigma_{a}^{i} m_{i} \rho_{a}^{i}+ \\
& \sum_{a=1}^{\mu}(p+c q) \mu_{a} \rho_{a}+\sum_{a \geq \mu+1} X_{j_{i}} \sigma_{a}(p+c q) \rho_{a}
\end{aligned}
$$

Hence, we get, for $\delta=\sum_{a=1}^{\beta} \mu_{a}^{i} \rho_{a}^{i}$ and $\nu=\sum_{a=1}^{\mu} \mu_{a} \rho_{a}$,

$$
\bar{\gamma} \equiv \overline{X_{s} \delta+\left(X_{j_{k}}+c X_{j_{l}}\right) \nu}
$$

Now that we have a better idea of the elemental structure of the ideal, we begin describing the linear maps of the field resolution.

Proposition 4.2 For $I=\left\langle m_{1}, \ldots, m_{n}, p+c q\right\rangle$ an ideal in $K\left\langle X_{1}, \ldots, X_{t}\right\rangle$ with $m_{s}=X_{j_{s}}^{2}$ for all $s$, and $p+c q=X_{j_{i}} X_{j_{k}}+c X_{j_{i}} X_{j_{l}}$, let $\varphi_{1}: \Lambda^{t} \longrightarrow \Lambda$ be defined by $\varphi_{1}=\left(\begin{array}{lll}X_{1} & \cdots & X_{t}\end{array}\right)$.

If $\left(\begin{array}{c}\overline{\gamma_{1}} \\ \overline{\gamma_{2}} \\ \vdots \\ \overline{\gamma_{t}}\end{array}\right) \in \operatorname{ker} \varphi_{1}$, then for some $\delta_{s}, \delta, \nu$,

$$
\overline{\gamma_{s}} \equiv \begin{cases}\overline{0} & \text { if } s \in\{1, \ldots, t\} \backslash\left\{j_{1}, \ldots, j_{n}\right\} \\ \overline{X_{s} \delta_{s}} & \text { if } s \in\left\{j_{1}, \ldots, j_{n}\right\} \backslash\left\{j_{i}\right\} \\ \hline X_{j_{i}} \delta+\left(X_{j_{k}}+c X_{j_{l}}\right) \nu & \text { if } s=j_{i}\end{cases}
$$

Proof Let $\left(\begin{array}{c}\overline{\gamma_{1}} \\ \vdots \\ \overline{\gamma_{t}}\end{array}\right) \in \operatorname{ker} \varphi_{1}$. Then, $\varphi_{1}\left(\begin{array}{c}\overline{\gamma_{1}} \\ \vdots \\ \overline{\gamma_{t}}\end{array}\right) \equiv \overline{0}$. Thus, $\sum_{a=1}^{t} X_{a} \gamma_{a} \in I$, and

$$
\sum_{a=1}^{t} X_{a} \gamma_{a}=\sum_{a=1}^{n} \beta_{a} m_{a} \lambda_{a}+\beta\left(X_{j_{i}} X_{j_{k}}+c X_{j_{i}} X_{j_{l}}\right) \lambda
$$

for some polynomials $\beta_{a}, \lambda_{a}, \beta, \lambda$. Rewriting:

$$
\sum_{a=1}^{t} X_{a} \gamma_{a}=\sum_{a=1}^{n_{1}} \mu_{a}^{1} m_{1} \rho_{a}^{1}+\sum_{a=1}^{n_{2}} \mu_{a}^{2} m_{2} \rho_{a}^{2}+\ldots+\sum_{a=1}^{n_{n}} \mu_{a}^{n} m_{n} \rho_{a}^{n}+\sum_{a=1}^{n^{\prime}} \mu_{a}\left(X_{j_{i}} X_{j_{k}}+c X_{j_{i}} X_{j_{l}}\right) \rho_{a}
$$


for some monomials $\mu_{a}^{j}, \rho_{a}^{j}, \mu_{a}, \rho_{a}$. Now, since for all s such that $\gamma_{s} \neq 0, X_{s}$ divides terms on the lefthand side of the equation, $X_{s}$ must divide terms on the righthand side on the equation:

$$
X_{s} \mid\left(\sum_{\substack{\text { subsum } \\ \text { over a }}} \mu_{a}^{1} m_{1} \rho_{a}^{1}+\ldots+\sum_{\substack{\text { subsum } \\ \text { over a }}} \mu_{a}^{n} m_{n} \rho_{a}^{n}+\sum_{\substack{\text { subsum } \\ \text { over a }}} \mu_{a}\left(X_{j_{i}} X_{j_{k}}+c X_{j_{i}} X_{j_{l}}\right) \rho_{a}\right)
$$

So,

$$
X_{s} \gamma_{s}=\sum_{j=1}^{n}\left(\sum_{\substack{\text { subsum } \\ \text { over a }}} \mu_{a}^{j} m_{j} \rho_{a}^{j}\right)+\sum_{\substack{\text { subsum } \\ \text { over a }}} \mu_{a}\left(X_{j_{i}} X_{j_{k}}+c X_{j_{i}} X_{j_{l}}\right) \rho_{a}
$$

Thus, $X_{s} \gamma_{s} \in I$, and so, by Proposition 4.1,

$$
\overline{\gamma_{s}} \equiv \overline{X_{s} \delta+\left(X_{j_{k}}+c X_{j_{l}}\right) \rho}
$$

where $\delta, \rho=0$ if $s \in\{1, \ldots, t\} \backslash\left\{j_{1}, \ldots, j_{n}\right\}$ and $\rho=0$ if $s \in\left\{j_{1}, \ldots, j_{n}\right\} \backslash\left\{j_{i}\right\}$

Proposition 4.3 For $I=\left\langle m_{1}, \ldots, m_{n}, p+c q\right\rangle$ an ideal in $K\left\langle X_{1}, \ldots, X_{t}\right\rangle$ with $m_{s}=X_{j_{s}}^{2}$ for all $1 \leq s \leq n$ and $p+c q=X_{j_{i}} X_{j_{k}}+c X_{j_{i}} X_{j_{l}}$, let $\varphi_{2}: \Lambda^{n+1} \longrightarrow \Lambda_{t}$ be defined by $\varphi_{2}=\left(a_{r s}\right)_{t \times(n+1)}$ where

$$
a_{r s}= \begin{cases}X_{j_{s}} & \text { if } r=j_{s} \text { and } 1 \leq s \leq i \\ X_{j_{k}}+c X_{j_{l}} & \text { if } r=j_{i} \text { and } s=i+1 \\ X_{j_{s-1}} & \text { if } r=j_{s-1} \text { and } i+2 \leq s \leq n+1 \\ 0 & \text { otherwise }\end{cases}
$$

(See Diagram 4.1)

If $\left(\begin{array}{c}\overline{\gamma_{1}} \\ \overline{\gamma_{2}} \\ \vdots \\ \overline{\gamma_{n+1}}\end{array}\right) \in \operatorname{ker} \varphi_{2}$, then for some polynomials $\delta_{s}, \delta, \xi$

$$
\overline{\gamma_{s}} \equiv \begin{cases}\overline{X_{j_{s}} \delta_{s}} & \text { if } 1 \leq s \leq i-1 \\ \overline{X_{j_{i}} \delta+\left(X_{j_{k}}+c X_{j_{l}}\right) \xi} & \text { if } s=i \\ \overline{0} & \text { if } s=i+1 \\ \overline{X_{j_{s-1}} \delta_{s-1}} & \text { if } i+2 \leq s \leq n+1\end{cases}
$$


Proof Let $\left(\begin{array}{c}\overline{\gamma_{1}} \\ \vdots \\ \overline{\gamma_{n+1}}\end{array}\right) \in \operatorname{ker} \varphi_{2}$. Then, $\varphi_{2}\left(\begin{array}{c}\overline{\gamma_{1}} \\ \vdots \\ \overline{\gamma_{n+1}}\end{array}\right) \equiv(\overline{0})_{t \times 1}$ Thus, $\left(\sum_{a=1}^{n+1} a_{r s} \gamma_{s}\right) \equiv(\overline{0})_{t \times 1}$.

For $\nu=1, \ldots, i-1$,

$$
X_{j_{\nu}} \gamma_{\nu}=\sum_{s=1}^{n+1} a_{j_{\nu} s} \gamma_{s} \in I
$$

So, by proposition $1, \overline{\gamma_{\nu}} \equiv \overline{X_{j_{\nu}} \delta_{\nu}}$ for some $\delta_{\nu}$. And, for $\nu=i+1, \ldots, n$,

$$
X_{j_{\nu}} \gamma_{\nu+1}=\sum_{s=1}^{n+1} a_{j_{\nu} s} \gamma_{s} \in I
$$

So, $\overline{\gamma_{\nu+1}} \equiv \overline{X_{j_{\nu}} \delta_{\nu+1}}$ for some $\delta_{\nu+1}$. Lastly, we also have that

$$
X_{j_{i}} \gamma_{i}+\left(X_{j_{k}}+c X_{j_{l}}\right) \gamma_{i+1}=\sum_{s=1}^{n+1} a_{j_{i} s} \gamma_{s} \in I
$$

Hence,

$$
X_{j_{i}} \gamma_{i}+X_{j_{k}} \gamma_{i+1}+c X_{j_{l}} \gamma_{i+1}=\sum_{s=1}^{n}\left(\sum_{a=1}^{n_{s}} \mu_{a}^{s} m_{s} \rho_{a}^{s}\right)+\sum_{a=1}^{n^{\prime}} \mu_{a}\left(X_{j_{i}} X_{j_{k}}+c X_{j_{i}} X_{j_{l}}\right) \rho_{a}
$$

for some monomials $\mu_{a}^{s}, \rho_{a}^{s}, \mu_{a}, \rho_{a}$. Thus, as in the proof of Proposition 4.2, we can write

$$
X_{j_{i}} \gamma_{i}=\sum_{s=1}^{n}\left(\sum_{\substack{\text { subsum } \\ \text { over a }}} \mu_{a}^{s} m_{s} \rho_{a}^{s}\right)+\sum_{\substack{\text { subsum } \\ \text { over a }}} \mu_{a}\left(X_{j_{i}} X_{j_{k}}+c X_{j_{i}} X_{j_{l}}\right) \rho_{a}
$$

So, $X_{j_{i}} \gamma_{i} \in I$. So, $\overline{\gamma_{i}} \equiv \overline{X_{j_{i}} \delta+\left(X_{j_{k}}+c X_{j_{l}}\right) \mu}$ for some $\delta, \mu$. Similarly, $X_{j_{k}} \gamma_{i+1}, X_{j_{l}} \gamma_{i+1} \in I$ which means $\overline{\gamma_{i+1}} \equiv \overline{X_{j_{k}} \delta^{\prime}}$ and $\overline{\gamma_{i+1}} \equiv \overline{X_{j_{l}} \delta^{\prime \prime}}$ for some $\delta^{\prime}, \delta^{\prime \prime}$. The only way this can occur is if $\overline{X_{j_{k}} \delta^{\prime}} \equiv \overline{0} \equiv \overline{X_{j_{l}} \delta^{\prime \prime}}$ Hence, $\overline{\gamma_{i+1}} \equiv \overline{0}$

Proposition 4.4 For $I=\left\langle m_{1}, \ldots, m_{n}, p+c q\right\rangle$ an ideal in $K\left\langle X_{1}, \ldots, X_{t}\right\rangle$ with $m_{s}=X_{j_{s}}^{2}$ for all $s$ and $p+c q=X_{j_{i}} X_{j_{k}}+c X_{j_{i}} X_{j_{l}}$, let $\varphi: \Lambda^{n+1} \longrightarrow \Lambda^{n+1}$ be defined by $\varphi=\left(b_{r s}\right)_{(n+1) \times(n+1)}$ where

$$
b_{r s}= \begin{cases}X_{j_{r}} & \text { if } r=s \text { and } 1 \leq r \leq i \\ X_{j_{k}}+c X_{j_{l}} & \text { if } r=i \text { and } s=i+1 \\ X_{j_{r-1}} & \text { if } r=s \text { and } i+2 \leq r \leq n+1 \\ 0 & \text { otherwise }\end{cases}
$$


(See Diagram 4.1)

If $\left(\begin{array}{c}\overline{\gamma_{1}} \\ \overline{\gamma_{2}} \\ \vdots \\ \overline{\gamma_{n+1}}\end{array}\right) \in \operatorname{ker} \varphi$, then for some $\delta_{s}, \delta, \nu$

$$
\overline{\gamma_{s}} \equiv \begin{cases}\overline{X_{j_{s}} \delta_{s}} & \text { if } 1 \leq s \leq i-1 \\ \overline{X_{j_{i}} \delta+\left(X_{j_{k}}+c X_{j_{l}}\right) \nu} & \text { if } s=i \\ \overline{0} & \text { if } s=i+1 \\ \overline{X_{j_{s-1}} \delta_{s}} & \text { if } i+2 \leq s \leq n+1\end{cases}
$$

Proof Let $\left(\begin{array}{c}\overline{\gamma_{1}} \\ \vdots \\ \overline{\gamma_{n+1}}\end{array}\right) \in \operatorname{ker} \varphi$. Then, $\varphi\left(\begin{array}{c}\overline{\gamma_{1}} \\ \vdots \\ \overline{\gamma_{n+1}}\end{array}\right) \equiv(\overline{0})_{(n+1) \times 1}$. So, $\sum_{a=1}^{n+1} b_{r s} \gamma_{s} \in I$. For $s=1, \ldots, i-1$,

$$
X_{j_{s}} \gamma_{s}=\sum_{a=1}^{n+1} b_{s a} \gamma_{a} \in I
$$

So, by Proposition $4.1, \overline{\gamma_{s}} \equiv \overline{X_{j_{s}} \delta_{s}}$ for some $\delta_{s}$. And, for $s=i+2, \ldots, n+1$,

$$
X_{j_{s-1}} \gamma_{s}=\sum_{a=1}^{n+1} b_{s a} \gamma_{a} \in I
$$

So, $\overline{\gamma_{s}} \equiv \overline{X_{j_{s-1}} \delta_{s}}$ for some $\delta_{s}$. Lastly, we also have that

$$
X_{j_{i}} \gamma_{i}+\left(X_{j_{k}}+c X_{j_{l}}\right) \gamma_{i+1}=\sum_{a=1}^{n+1} b_{i a} \gamma_{a} \in I
$$

So, $X_{j_{i}} \gamma_{i} \in I$ and $\overline{\gamma_{i}} \equiv \overline{X_{j_{i}} \delta+\left(X_{j_{k}}+c X_{j_{l}}\right) \mu}$ for some $\delta$, $\mu$. And, $X_{j_{k}} \gamma_{i+1}, X_{j_{l}} \gamma_{i+1} \in I$. Thus, $\overline{\gamma_{i+1}} \equiv \overline{X_{j_{k}} \delta^{\prime}}$ and $\overline{\gamma_{i+1}} \equiv \overline{X_{j_{l}} \delta^{\prime \prime}}$ for some $\delta^{\prime}, \delta^{\prime \prime}$. Hence,

$$
\overline{X_{j_{k}} \delta^{\prime}} \equiv \overline{0} \equiv \overline{X_{j_{l}} \delta^{\prime \prime}}
$$

Hence, $\overline{\gamma_{i+1}} \equiv \overline{0}$

Theorem 4.1 Let $I=\left\langle m_{1}, \ldots, m_{n}, p+c q\right\rangle$ be an ideal in $K\left\langle X_{1}, \ldots, X_{t}\right\rangle$ where $m_{s}=X_{j_{s}}^{2}$ for all $s$, and $p+c q=X_{j_{i}} X_{j_{k}}+c X_{j_{i}} X_{j_{l}}$ for some distinct $i, k, l \in\{1, \ldots, n\}$. Then, $\Lambda$ has the following linear resolution:

$$
(*) \cdots \stackrel{\varphi}{\rightarrow} \Lambda^{n+1} \stackrel{\varphi}{\rightarrow} \Lambda^{n+1} \stackrel{\varphi}{\rightarrow} \Lambda^{n+1} \stackrel{\varphi_{2}}{\rightarrow} \Lambda^{t} \stackrel{\varphi_{1}}{\rightarrow} \Lambda \rightarrow k \rightarrow 0
$$

where $\varphi, \varphi_{1}$, and $\varphi_{2}$ are as defined in Propositions 4.2, 4.3, 4.4. 
Proof First look at $\varphi_{1} \circ \varphi_{2}=\left(\sum_{r=1}^{t} X_{r} a_{r s}\right)$

For $1 \leq s \leq i, \sum_{r=1}^{t} X_{r} a_{r s}=\overline{X_{j_{s}}^{2}} \equiv \overline{0}$.

For $s=i+1, \sum_{r=1}^{t} X_{r} a_{r s}=X_{j_{i}}\left(\overline{\left.X_{j_{k}}+c X_{j_{l}}\right)} \equiv \overline{X_{j_{i}} X_{j_{k}}+c X_{j_{i}} X_{j_{l}}} \equiv \overline{0}\right.$.

For $i+2 \leq s \leq n+1, \sum_{r=1}^{t} X_{r} a_{r s}=\overline{X_{j_{s-1}}^{2}} \equiv \overline{0}$.

Hence, $\varphi_{1} \circ \varphi_{2}=(0)_{1 \times(n+1)}$ and $\operatorname{Im} \varphi_{2} \subseteq \operatorname{ker} \varphi_{1}$.

Let $\left(\begin{array}{c}\overline{\gamma_{1}} \\ \vdots \\ \overline{\gamma_{t}}\end{array}\right) \in \operatorname{ker} \varphi_{1}$. Then, by Proposition 4.2,

$$
\overline{\gamma_{j}}= \begin{cases}\overline{0} & \text { if } j \in\{1, \ldots, t\} \backslash\left\{j_{1}, \ldots, j_{n}\right\} \\ \overline{X_{j} \delta_{j}} & \text { if } \left.j \in\left\{j_{1}, \ldots, j_{n}\right\} \backslash j_{i}\right\} \\ \overline{X_{j_{i}} \delta+\left(X_{j_{k}}+c X_{j_{l}}\right) \nu} & \text { if } j=j_{i}\end{cases}
$$

for some $\delta_{j}, \delta, \nu$. Then,

$$
\varphi_{2}\left(\begin{array}{c}
\overline{\delta_{j_{1}}} \\
\vdots \\
\overline{\delta_{j_{i-1}}} \\
\bar{\nu} \\
\overline{\delta_{j_{i+1}}} \\
\vdots \\
\overline{\delta_{j_{n}}}
\end{array}\right)=\left(\begin{array}{c}
\overline{\gamma_{1}} \\
\vdots \\
\overline{\gamma_{t}}
\end{array}\right)
$$

So, $\operatorname{ker} \varphi_{1}=\operatorname{Im} \varphi_{2}$.

Next, look at $\varphi_{2} \circ \varphi=\left(a_{r s}\right)\left(b_{r s}\right)=\left(\sum_{s=1}^{n+1} a_{r s} b_{s w}\right)$.

For $r=j_{v}, v \neq i$ and $w=v, \sum a_{r s} b_{s w}=a_{j_{v} v} b_{v v}=\overline{X_{j_{v}}^{2}} \equiv \overline{0}$.

For $r=j_{i}$ and $w=i, \sum a_{r s} b_{s w}=a_{j_{i}} b_{i i}=\overline{X_{j_{i}}^{2}} \equiv \overline{0}$.

For $r=j_{i}$ and $w=i+1, \sum a_{r s} b_{s w}=a_{j_{i}} b_{i(i+1)}=\overline{X_{j_{i}}\left(X_{j_{k}}+c X_{j_{l}}\right)} \equiv \overline{0}$.

For all other $\mathrm{r}$ and $\mathrm{w}, \sum a_{r s} b_{s w}=0$.

So, $\varphi_{2} \circ \varphi \equiv(0)_{t \times(n+1)}$ and $\operatorname{Im} \varphi_{2} \subseteq \operatorname{ker} \varphi$. 
Let $\left(\begin{array}{c}\overline{\gamma_{1}} \\ \vdots \\ \overline{\gamma_{n+1}}\end{array}\right) \in \operatorname{ker} \varphi_{2}$. Then, by Proposition 4.3,

$$
\overline{\gamma_{s}}= \begin{cases}\overline{X_{j_{s}} \delta_{s}} & \text { if } 1 \leq s \leq i-1 \\ \overline{X_{j_{i}} \delta+\left(X_{j_{k}}+c X_{j_{l}}\right) \nu} & \text { if } s=i \\ \overline{0} & \text { if } s=i+1 \\ \overline{X_{j_{s-1}} \delta_{s-1}} & \text { if } i+2 \leq s \leq n+1\end{cases}
$$

for some $\delta_{s}, \delta, \nu$. And,

$$
\varphi\left(\begin{array}{c}
\overline{\delta_{1}} \\
\vdots \\
\overline{\delta_{i-1}} \\
\bar{\delta} \\
\bar{\nu} \\
\overline{\delta_{i+1}} \\
\vdots \\
\overline{\delta_{n}}
\end{array}\right)=\left(\begin{array}{c}
\overline{\gamma_{1}} \\
\vdots \\
\overline{\gamma_{n+1}}
\end{array}\right)
$$

Hence, $\operatorname{ker} \varphi_{2}=\operatorname{Im} \varphi$.

Lastly, $\varphi \circ \varphi=\left(\sum_{v=1}^{n+1} b_{r v} b_{v s}\right)$.

For $r \neq i, i+1$ and $s=r$,

$$
\sum b_{r v} b_{v s}=b_{r r} b_{r r}=\left\{\begin{array}{ll}
\overline{X_{j_{r}}^{2}} & \text { if } 1 \leq r \leq i-1 \\
\overline{X_{j_{r-1}}^{2}} & \text { if } i+2 \leq r \leq n+1
\end{array}\right\} \equiv \overline{0}
$$

For $r, s=i, \sum b_{r v} b_{v s}=b_{i i} b_{i i}=\overline{X_{j_{i}}^{2}} \equiv \overline{0}$.

For $r=i$ and $s=i+1, \sum b_{r v} b_{v s}=b_{i i} b_{i(i+1)}=\overline{X_{j_{i}}\left(X_{j_{k}}+c X_{j_{l}}\right)} \equiv \overline{0}$.

For all other $\mathrm{r}$ and $\mathrm{s}, \sum b_{r v} b_{v s}=0$.

Hence, $\varphi \circ \varphi \equiv(0)_{(n+1) \times(n+1)}$ and $\operatorname{Im} \varphi \subseteq \operatorname{ker} \varphi$.

Let $\left(\begin{array}{c}\overline{\gamma_{1}} \\ \vdots \\ \overline{\gamma_{n+1}}\end{array}\right) \in \operatorname{ker} \varphi$. Then, by Proposition 4.4,

$$
\overline{\gamma_{s}}= \begin{cases}\overline{X_{j_{s}} \delta_{s}} & \text { if } 1 \leq s \leq i-1 \\ \overline{X_{j_{i}} \delta+\left(X_{j_{k}}+c X_{j_{l}}\right) \nu} & \text { if } s=i \\ \overline{0} & \text { if } s=i+1 \\ \overline{X_{j_{s-1}} \delta_{s}} & \text { if } i+2 \leq s \leq n+1\end{cases}
$$


for some $\delta_{s}, \delta, \nu$. Then,

$$
\varphi\left(\begin{array}{c}
\overline{\delta_{1}} \\
\vdots \\
\overline{\delta i-1} \\
\bar{\delta} \\
\bar{\nu} \\
\overline{\delta_{i+1}} \\
\vdots \\
\overline{\delta_{n+1}}
\end{array}\right)=\left(\begin{array}{c}
\overline{\gamma_{1}} \\
\vdots \\
\overline{\gamma_{n+1}}
\end{array}\right)
$$

Thus, $\operatorname{ker} \varphi=\operatorname{Im} \varphi$.

Hence, $(*)$ is exact.

So, here we see our first example of a quadratic algebra having no quadratic Gröbner basis which is nonetheless a Koszul algebra. The symmetric case, $I=\left\langle m_{1}, \ldots, m_{n}, X_{j_{k}} X_{j_{i}}+\right.$ $\left.c X_{j_{l}} X_{j_{i}}\right\rangle$, while similar in nature, has its own set of idiosyncracies. As before, we start with an investigation of ideal membership.

Proposition 4.5 Let $I=\left\langle m_{1}, \ldots, m_{n}, p+c q\right\rangle$ be an ideal in $K\left\langle X_{1}, \ldots, X_{t}\right\rangle$, with $p+c q=$ $X_{j_{k}} X_{j_{i}}+c X_{j_{l}} X_{j_{i}}$ for $k \neq i, k \neq l, i \neq l$, If $X_{j_{v}} \gamma \in I, v \neq k, l$, then $\bar{\gamma} \equiv \overline{X_{j_{v}} \delta}$ for some $\delta$. Futhermore, if $X_{s} \gamma \in I$, for $s \neq j_{v}$ for all $v$, then $\gamma \in I$.

Proof Suppose $X_{j_{v}} \gamma \in I$ for some $v \neq k, l$. Then, for some monomials $\mu_{j}^{a}, \rho_{j}^{a}, \mu_{j}, \rho_{j}$,

$$
X_{j_{v}} \gamma=\sum_{a=1}^{n}\left(\sum_{j=1}^{n_{a}} \mu_{j}^{a} m_{a} \rho_{j}^{a}\right)+\sum_{j=1}^{n_{\text {sum }}} \mu_{j}\left(X_{j_{k}} X_{j_{i}}+c X_{j_{l}} X_{j_{i}}\right) \rho_{j}
$$

Since $v \neq k, l$, we have that $\mu_{j} \notin K \forall j=1, \ldots, n_{\text {sum }}$, and so, $\mu_{j}=X_{j_{k}} \sigma_{j}$ for some monomial $\sigma_{j}$. Similarly, for all $\mathrm{j}$ and $a \neq v, \mu_{j}^{a}=X_{j_{k}} \sigma_{j}^{a}$ for some monomial $\sigma_{j}^{a}$.

$$
\begin{gathered}
\text { Thus, } X_{j_{v}} \gamma=\sum_{a \neq v}\left(\sum_{j=1}^{n_{a}} X_{j_{v}} \sigma_{j}^{a} m_{a} \rho_{j}^{a}\right)+\sum_{\substack{\text { subsum } \\
\text { over j }}} X_{j_{v}} \sigma_{j}^{v} m_{v} \rho_{j}^{v} \\
+\sum_{\substack{\text { subsum } \\
\text { over j }}} \mu_{j}^{v} X_{j_{v}}^{2} \rho_{j}^{v}+\sum_{j=1}^{n_{\text {sum }}} X_{j_{v}} \sigma_{j}\left(X_{j_{k}} X_{j_{i}}+c X_{j_{l}} X_{j_{i}}\right) \rho_{j}
\end{gathered}
$$




$$
\text { So, } \bar{\gamma}=\overline{X_{j_{v}} \delta} \text { for } \delta=\sum \mu_{j}^{v} \rho_{j}^{v}
$$

And, if $X_{s} \gamma \in I, s \neq j_{v}$ for all $\mathrm{v}$, then, for some monomials $\mu_{j}^{a}, \rho_{j}^{a}, \mu_{j}, \rho_{j}$,

$$
X_{s} \gamma=\sum_{a=1}^{n}\left(\sum_{j=1}^{n_{a}} \mu_{j}^{a} m_{a} \rho_{j}^{a}\right)+\sum_{j=1}^{n_{s u m}} \mu_{j}\left(X_{j_{k}} X_{j_{i}}+c X_{j_{l}} X_{j_{i}}\right) \rho_{j}
$$

But, since $s \neq j_{v} \forall v, \mu_{j}^{a}=X_{s} \sigma_{j}^{a} \forall a, j$, and $\mu_{j}=X_{s} \sigma_{j} \forall j$ for some monomials $\sigma_{j}^{a}, \sigma_{j}$. Hence, $\bar{\gamma} \equiv \overline{0}$.

We now begin delineating the maps of the linear resolution of the field.

Proposition 4.6 For $p+c q=X_{j_{k}} X_{j_{i}}+c X_{j_{l}} X_{j_{i}}$, where $i, j, k$ are distinct, let $\varphi_{1}: \Lambda^{t} \rightarrow \Lambda$ be defined by $\varphi_{1}=\left(X_{1} \cdots X_{t}\right)$. Then,

$$
\operatorname{ker} \varphi_{1}=\left\{\left(\begin{array}{c}
\overline{\gamma_{1}} \\
\vdots \\
\overline{\gamma_{t}}
\end{array}\right) \mid \overline{\gamma_{s}} \equiv\left\{\begin{array}{ll}
\overline{0} & \text { if } s \in\{1, \ldots, t\} \backslash\left\{j_{1}, \ldots, j_{n}\right\} \\
\overline{X_{s} \delta_{s}} & \text { if } s \in\left\{j_{1}, \ldots, j_{n}\right\} \backslash\left\{j_{k}, j_{l}\right\} \\
\overline{X_{j_{k}} \delta_{j_{k}}+X_{j_{i}} \nu} & \text { if } s=j_{k} \\
\hline X_{j_{l}} \delta_{j_{l}}+c X_{j_{i}} \nu & \text { if } s=j_{l}
\end{array}\right\}\right.
$$

for some $\delta_{1}, \ldots, \delta_{t}, \nu$.

Proof Let $\left(\begin{array}{c}\overline{\gamma_{1}} \\ \vdots \\ \overline{\gamma_{t}}\end{array}\right) \in \operatorname{ker} \varphi_{1}$. Then, $\varphi_{1}\left(\begin{array}{c}\overline{\gamma_{1}} \\ \vdots \\ \overline{\gamma_{t}}\end{array}\right)=\sum_{s=1}^{t} X_{s} \gamma_{s} \in I$.

$$
\text { And, } \sum_{s=1}^{t} X_{s} \gamma_{s}=\sum_{a=1}^{n}\left(\sum_{j=1}^{n_{a}} \mu_{j}^{a} m_{a} \rho_{j}^{a}\right)+\sum_{j=1}^{n_{s u m}} \mu_{j}\left(X_{j_{k}} X_{j_{i}}+c X_{j_{l}} X_{j_{i}}\right) \rho_{j}
$$

for some monomials $\mu_{j}^{a}, \mu_{j}, \rho_{j}^{a}, \rho_{j}$. So, for $s \neq j_{v} \forall v$,

$$
X_{s} \gamma_{s}=\underbrace{\sum_{a=1}^{n}\left(\sum_{\substack{\text { subsum } \\
\text { over j }}} \mu_{j}^{a} m_{a} \rho_{j}^{a}\right)+\sum_{\begin{array}{c}
\text { subsum } \\
\text { over j }
\end{array}} \mu_{j}\left(X_{j_{k}} X_{j_{i}}+c X_{j_{l}} X_{j_{i}}\right) \rho_{j}}_{\text {divisible by } X_{s}}
$$


Thus, $X_{s} \gamma_{s} \in I$ and $\overline{\gamma_{s}} \equiv \overline{0}$. For $s=j_{v}, v \neq k, l$,

$$
X_{j_{v}} \gamma_{j_{v}}=\underbrace{\sum_{a=1}^{n}\left(\sum_{\substack{\text { subsum } \\ \text { over j }}} \mu_{j}^{a} m_{a} \rho_{j}^{a}\right)+\sum_{\substack{\text { subsum } \\ \text { over j }}} \mu_{j}\left(X_{j_{k}} X_{j_{i}}+c X_{j_{l}} X_{j_{i}}\right) \rho_{j}}
$$

divisible by $X_{j_{v}}$

Then, $X_{j_{v}} \gamma_{j_{v}} \in I$ and $\overline{\gamma_{j_{v}}} \equiv \overline{X_{j_{v}} \delta_{j_{v}}}$ for some $\delta_{j_{v}}$ And,

$$
\begin{aligned}
& X_{j_{k}} \gamma_{j_{k}}=\sum_{a=1}^{n}\left(\sum_{\substack{\text { subsum } \\
\text { over j }}} \mu_{j}^{a} m_{a} \rho_{j}^{a}\right)+\sum_{\substack{\text { subsum } \\
\text { over j }}} \overbrace{\mu_{j}}^{\notin K}\left(X_{j_{k}} X_{j_{i}}+c X_{j_{l}} X_{j_{i}}\right) \rho_{j} \\
& +\sum_{\substack{\text { subsum } \\
\text { over a }}} \overbrace{\mu_{j}}^{\in K} X_{j_{k}} X_{j_{i}} \rho_{j} \\
& X_{j_{l}} \gamma_{j_{l}}=\sum_{a=1}^{n}\left(\sum_{\substack{\text { subsum } \\
\text { over j }}} \mu_{j}^{a} m_{a} \rho_{j}^{a}\right)+\sum_{\substack{\text { subsum } \\
\text { over j }}} \overbrace{\mu_{j}}^{\notin K}\left(X_{j_{k}} X_{j_{i}}+c X_{j_{l}} X_{j_{i}}\right) \rho_{j} \\
& +\sum_{\substack{\text { subsum } \\
\text { over } \mathrm{j}}} \overbrace{\mu_{j}}^{\in K} c X_{j_{l}} X_{j_{i}} \rho_{j}
\end{aligned}
$$

Now, if $\mu_{j} X_{j_{k}} X_{j_{i}} \rho_{j}$ is a term in $\sum \mu_{j} X_{j_{k}} X_{j_{i}} \rho_{j}$, then its pair, $c \mu_{j} X_{j_{l}} X_{j_{i}} \rho_{j}$, either appears as a term in $\sum \mu_{j} c X_{j_{l}} X_{j_{i}} \rho_{j}$ in $X_{j_{l}} \gamma_{j_{l}}$, or it cancels with a $\mu_{j^{\prime}}^{a} m_{a} \rho_{j^{\prime}}^{a}$ term in the original sum. Hence, either $a=i$ and $\rho_{j}=X_{j_{i}} \rho_{j^{\prime}}^{a}$ or $m_{a} \mid \rho_{j}$. Similarly, if $\mu_{j} c X_{j_{l}} X_{j_{i}} \rho_{j}$ is a term in $\sum \mu_{j} c X_{j_{l}} X_{j_{i}} \rho_{j}$, then its pair, $\mu_{j} X_{j_{k}} X_{j_{i}} \rho_{j}$, either appears as a term in $\sum \mu_{j} X_{j_{k}} X_{j_{i}} \rho_{j}$ in $X_{j_{k}} \gamma_{j_{k}}$, or it cancels with a $\mu_{j^{\prime \prime}}^{a} m_{a} \rho_{j^{\prime \prime}}^{a}$ term in the original sum. Thus, either $a=i$ and $\rho_{j}=X_{j_{i}} \rho_{j^{\prime \prime}}^{a}$ or $m_{a} \mid \rho_{j}$. Hence,

$$
X_{j_{k}} \gamma_{j_{k}}=\sum_{a \neq k}\left(\sum_{\substack{\text { subsum } \\ \text { over j }}} X_{j_{k}} \sigma_{j}^{a} m_{a} \rho_{j}^{a}\right)+\sum_{\substack{\text { subsum } \\ \text { over j }}} X_{j_{k}} \sigma_{j}^{k} m_{k} \rho_{j}^{k}
$$




$$
\begin{aligned}
& +\sum_{\substack{\text { subsum } \\
\text { over j }}} \overbrace{\mu_{j}^{k}}^{\in K} X_{j_{k}}^{2} \rho_{j}^{k}+\sum_{\substack{\text { subsum } \\
\text { over j }}} X_{j_{k}} \sigma_{j}\left(X_{j_{k}} X_{j_{i}}+c X_{j_{l}} X_{j_{i}}\right) \rho_{j} \\
& +\sum_{m_{a} \mid \rho_{j}} \mu_{j} X_{j_{k}} X_{j_{i}} \rho_{j}+\sum \mu_{j} X_{j_{k}} X_{j_{i}}^{2} \rho_{j}^{i}+\sum_{\text {pair in } X_{j_{l}} \gamma_{j_{l}}} \mu_{j} X_{j_{k}} X_{j_{i}} \rho_{j}
\end{aligned}
$$

And,

$$
\begin{aligned}
& X_{j_{l}} \gamma_{j_{l}}=\sum_{a \neq l}\left(\sum_{\substack{\text { subsum } \\
\text { over j }}} X_{j_{l}} \sigma_{j}^{a} m_{a} \rho_{j}^{a}\right)+\sum_{\substack{\text { subsum } \\
\text { over j }}} X_{j_{l}} \sigma_{j}^{l} m_{l} \rho_{j}^{l} \\
& +\sum_{\substack{\text { subsum } \\
\text { over } \mathrm{j}}} \overbrace{\mu_{j}^{l}}^{\in K} X_{j_{l}}^{2} \rho_{j}^{l}+\sum_{\substack{\text { subsum } \\
\text { over } \mathrm{j}}} X_{j_{l}} \sigma_{j}\left(X_{j_{k}} X_{j_{i}}+c X_{j_{l}} X_{j_{i}}\right) \rho_{j} \\
& +\sum_{m_{a} \mid \rho_{j}} \mu_{j} c X_{j_{l}} X_{j_{i}} \rho_{j}+\sum \mu_{j} c X_{j_{l}} X_{j_{i}}^{2} \rho_{j}^{i}+\sum_{\text {pair in } X_{j_{k}} \gamma_{j_{k}}} \mu_{j} c X_{j_{l}} X_{j_{i}} \rho_{j}
\end{aligned}
$$

So,

$$
\begin{gathered}
\overline{\gamma_{j_{k}}} \equiv \overline{X_{j_{k}} \delta_{j_{k}}+X_{j_{i}} \nu} \\
\overline{\gamma_{j_{l}}} \equiv \overline{X_{j_{l}} \delta_{j_{l}}+c X_{j_{i}} \nu}
\end{gathered}
$$

for $\delta_{j_{k}}=\sum \mu_{j}^{k} \rho_{j}^{k}, \delta_{j_{l}}=\sum \mu_{j}^{l} \rho_{j}^{l}$, and $\nu=\sum$ over pairs $\mu_{j} \rho_{j}$.

Proposition 4.7 For $I=\left\langle m_{1}, \ldots, m_{n}, p+c q\right\rangle$ an ideal in $K\left\langle X_{1}, \ldots, X_{t}\right\rangle$ with $m_{s}=X_{j_{s}}^{2}$ for all $s$ and $p+c q=X_{j_{k}} X_{j_{i}}+c X_{j_{l}} X_{j_{i}}$, let $\varphi_{2}: \Lambda^{n+1} \rightarrow \Lambda^{t}$ be defined by $\varphi_{2}=\left(a_{r s}\right)$ where

$$
a_{r s}= \begin{cases}X_{j_{s}} & \text { if } r=j_{s} \text { and } 1 \leq s \leq n \\ c X_{j_{i}} & \text { if } r=j_{l} \text { and } s=n+1 \\ X_{j_{i}} & \text { if } r=j_{k} \text { and } s=n+1 \\ 0 & \text { if otherwise }\end{cases}
$$

(See Diagram 4.2)

Then,

$$
\operatorname{ker} \varphi_{2}=\left\{\left(\begin{array}{c}
\overline{X_{j_{1}} \delta_{1}} \\
\vdots \\
\frac{X_{j_{n}} \delta_{n}}{X_{j_{i}} \delta_{n+1}}
\end{array}\right) \mid \delta_{s} \in K\left\langle X_{1}, \ldots, X_{t}\right\rangle \text { for } 1 \leq s \leq n+1\right\}
$$


Proof Let $\left(\begin{array}{c}\overline{\gamma_{1}} \\ \vdots \\ \overline{\gamma_{n+1}}\end{array}\right) \in \operatorname{ker} \varphi_{2}$ Then, $\varphi_{2}\left(\begin{array}{c}\overline{\gamma_{1}} \\ \vdots \\ \overline{\gamma_{n+1}}\end{array}\right)=\left(\sum_{s=1}^{n+1} a_{r s} \overline{\gamma_{s}}\right) \equiv(\overline{0})$. And,

$$
\sum_{s=1}^{n+1} a_{r s} \gamma_{s} \in I
$$

forall $r$. So, for $r=j_{v}, v \neq k, l$,

$$
\sum_{s=1}^{n+1} a_{j_{v}} \gamma_{s}=a_{j_{v}} \gamma_{v}=X_{j_{v}} \gamma_{v} \in I \text { and } \overline{\gamma_{v}} \equiv \overline{X_{j_{v}} \delta_{v}} \text { for some } \delta_{v}
$$

And, for $r=j_{k}, j_{l}$,

$$
\sum_{s=1}^{n+1} a_{j_{s} s} \gamma_{s}=a_{j_{k} k} \gamma_{k}+a_{j_{k} n+1} \gamma_{n+1}=X_{j_{k}} \gamma_{k}+X_{j_{i}} \gamma_{n+1} \in I
$$

Thus, $\sum_{s=1}^{n+1} a_{j_{l} s} \gamma_{s}=a_{j_{l}} \gamma_{l}+a_{j_{l} n+1} \gamma_{n+1}=X_{j_{l}} \gamma_{l}+c X_{j_{i}} \gamma_{n+1} \in I$

Now, $X_{j_{k}} \gamma_{k}+X_{j_{i}} \gamma_{n+1} \in I$

$$
\text { So, } X_{j_{k}} \gamma_{k}+X_{j_{i}} \gamma_{n+1}=\sum_{a=1}^{n}\left(\sum_{j=1}^{n_{a}} \mu_{j}^{a} m_{a} \rho_{j}^{a}\right)+\sum_{j=1}^{n_{s u m}} \mu_{j}\left(X_{j_{k}} X_{j_{i}}+c X_{j_{l}} X_{j_{i}}\right) \rho_{j}
$$

for some monomials $\mu_{j}^{a}, \rho_{j}^{a}, \mu_{j}, \rho_{j}$.

$$
\begin{gathered}
\text { And, } X_{j_{k}} \gamma_{k}=\sum_{a=1}^{n}\left(\sum_{\substack{\text { subsum } \\
\text { over j }}} \mu_{j}^{a} m_{a} \rho_{j}^{a}\right)+\sum_{\substack{\text { subsum } \\
\text { over j }}} \overbrace{\mu_{j}}^{\notin K}\left(X_{j_{k}} X_{j_{i}}+c X_{j_{l}} X_{j_{i}}\right) \rho_{j} \\
+\sum_{\substack{\text { subsum } \\
\text { over j }}} \overbrace{\mu_{j}}^{\in K} X_{j_{k}} X_{j_{i}} \rho_{j}
\end{gathered}
$$

As in the proof of Proposition 4.6 we get,

$$
\begin{aligned}
& X_{j_{k}} \gamma_{k}=\sum_{a \neq k}\left(\sum X_{j_{k}} \sigma_{j}^{a} m_{a} \rho_{j}^{a}\right)+\sum X_{j_{k}} \sigma_{j}^{k} m_{k} \rho_{j}^{k}+\sum \overbrace{\mu_{j}^{k}}^{\in K} X_{j_{k}}^{2} \rho_{j}^{k} \\
& +\sum X_{j_{k}} \sigma_{j}\left(X_{j_{k}} X_{j_{i}}+c X_{j_{l}} X_{j_{i}}\right) \rho_{j}+\sum_{m_{a} \mid \rho_{j} \text { for some a }} \mu_{j} X_{j_{k}} X_{j_{i}} \rho_{j}
\end{aligned}
$$


H. C. McGilvray

$$
+\sum \mu_{j} X_{j_{k}} X_{j_{i}}^{2} \rho_{j^{\prime}}^{a}
$$

Thus, $\overline{\gamma_{k}} \equiv \overline{X_{j_{k}} \delta_{k}}$ for $\delta_{k}=\sum \mu_{j}^{k} \rho_{j}^{k}$

And,

$$
X_{j_{i}} \gamma_{n+1} \in I \text { which implies } \overline{\gamma_{n+1}} \equiv \overline{X_{j_{i}} \delta_{n+1}} \text { for some } \delta_{n+1}
$$

Similarly,

$$
\begin{gathered}
X_{j_{l}} \gamma_{l}+c X_{j_{i}} \gamma_{n+1} \in I \\
\text { and } \overline{\gamma_{l}} \equiv \overline{X_{j_{l}} \delta_{l}} \text { for } \delta_{l}=\sum \mu_{j}^{l} \rho_{j}^{l}
\end{gathered}
$$

Proposition 4.8 For $I=\left\langle m_{1}, \ldots, m_{n}, p+c q\right\rangle$ an ideal in $K\left\langle X_{1}, \ldots, X_{t}\right\rangle$ with $m_{s}=X_{j_{s}}^{2}$ for all $s$ and $p+c q=X_{j_{k}} X_{j_{i}}+c X_{j_{l}} X_{j_{i}}$, let $\varphi: \Lambda^{n+1} \rightarrow \Lambda^{n+1}$ be defined by $\varphi=\left(b_{r s}\right)$ where

$$
b_{r s}= \begin{cases}X_{j_{r}} & \text { if } r=s \text { and } 1 \leq s \leq n \\ X_{j_{i}} & \text { if } r=s=n+1 \\ 0 & \text { if otherwise }\end{cases}
$$

(See Diagram 4.2)

Then,

$$
\operatorname{ker} \varphi=\left\{\left(\begin{array}{c}
\overline{X_{j_{1}} \delta_{1}} \\
\vdots \\
\frac{X_{j_{n}} \delta_{n}}{X_{j_{i}} \delta_{n+1}}
\end{array}\right) \mid \delta_{s} \in\left\langle X_{1}, \ldots, X_{t}\right\rangle \text { for } s=1, \ldots, n+1\right\}
$$

Proof Let $\left(\begin{array}{c}\overline{\gamma_{1}} \\ \vdots \\ \hline \overline{\gamma_{n+1}}\end{array}\right) \in \operatorname{ker} \varphi$

Then, $\varphi\left(\begin{array}{c}\overline{\gamma_{1}} \\ \vdots \\ \overline{\gamma_{n+1}}\end{array}\right)=\left(\sum_{s=1}^{n+1} b_{r s} \overline{\gamma_{s}}\right) \equiv(\overline{0})$. Hence, $\sum_{s=1}^{n+1} b_{r s} \gamma_{s} \in I \forall r$

So, for $1 \leq r \leq n, r \neq k, l$,

$$
\sum_{s=1}^{n+1} b_{r s} \gamma_{s}=b_{r r} \gamma_{r}=X_{j_{r}} \gamma_{r} \in I \text { and } \forall r \neq k, l, \overline{\gamma_{r}} \equiv \overline{X_{j_{r}} \delta_{r}} \text { for some } \delta_{r}
$$

And, for $r=n+1$, 


$$
\begin{gathered}
\sum_{s=1}^{n+1} b_{(n+1) s} \gamma_{s}=b_{(n+1)(n+1)} \gamma_{n+1}=X_{j_{i}} \gamma_{n+1} \in I . \\
\text { So, } \overline{\gamma_{n+1}} \equiv \overline{X_{j_{i}} \delta_{n+1}}
\end{gathered}
$$

for some $\delta_{n+1}$.

Now, since $X_{j_{k}} \gamma_{k} \in I$,

$$
X_{j_{k}} \gamma_{k}=\sum_{a=1}^{n}\left(\sum_{j=1}^{n_{a}} \mu_{j}^{a} m_{a} \rho_{j}^{a}\right)+\sum_{j=1}^{n_{\text {sum }}} \mu_{j}\left(X_{j_{k}} X_{j_{i}}+c X_{j_{l}} X_{j_{i}}\right) \rho_{j}
$$

for some monomials $\mu_{j}^{a}, \rho_{j}^{a}, \mu_{j}, \rho_{j}$.

$$
\begin{gathered}
\text { Hence, } X_{j_{k}} \gamma_{k}=\sum_{a=1}^{n}\left(\sum_{\begin{array}{c}
\text { subsum } \\
\text { over j }
\end{array}} \mu_{j}^{a} m_{a} \rho_{j}^{a}\right)+\sum_{\substack{\text { subsum } \\
\text { over j }}} \overbrace{\mu_{j}}^{\notin K}\left(X_{j_{k}} X_{j_{i}}+c X_{j_{l}} X_{j_{i}}\right) \rho_{j} \\
+\sum_{\substack{\text { subsum } \\
\text { over j }}} \overbrace{\mu_{j}}^{\in K} X_{j_{k}} X_{j_{i}} \rho_{j}
\end{gathered}
$$

As in the proof of Proposition 4.6,

$$
\begin{gathered}
X_{j_{k}} \gamma_{k}=\sum_{a \neq k}\left(\sum X_{j_{k}} \sigma_{j}^{a} m_{a} \rho_{j}^{a}\right)+\sum X_{j_{k}} \sigma_{j}^{k} m_{k} \rho_{j}^{k} \\
+\sum \overbrace{\mu_{j}^{k}}^{\in K} X_{j_{k}}^{2} \rho_{j}^{k}+\sum X_{j_{k}} \sigma_{j}\left(X_{j_{k}} X_{j_{i}}+c X_{j_{l}} X_{j_{i}}\right) \rho_{j} \\
+\sum_{m_{a} \mid \rho_{j} \text { for some a }} \mu_{j} X_{j_{k}} X_{j_{i}} \rho_{j}+\sum \mu_{j} X_{j_{k}} X_{j_{i}}^{2} \rho_{j^{\prime}}^{a} \\
\text { Thus, } \overline{\gamma_{k}} \equiv \overline{X_{j_{k}} \delta_{k}} \text { for } \delta_{k}=\sum \mu_{j}^{k} \rho_{j}^{k}
\end{gathered}
$$

Similarly, $X_{j_{l}} \gamma_{l} \in I$. And, $\overline{\gamma_{l}} \equiv \overline{X_{j_{l}} \delta_{l}}$ for $\delta_{l}=\sum \mu_{j}^{l} \rho_{j}^{l}$ 
Theorem 4.2 Let $I=\left\langle m_{1}, \ldots, m_{n}, p+c q\right\rangle$ be an ideal in $K\left\langle X_{1}, \ldots, X_{t}\right\rangle$ where $m_{s}=X_{j_{s}}^{2}$ for all $s$, and $p+c q=X_{j_{k}} X_{j_{i}}+c X_{j_{l}} X_{j_{i}}$ for some distinct $i, k, l \in\{1, \ldots, n\}$. Then, $\Lambda=$ $K\left\langle X_{1}, \ldots, X_{t}\right\rangle / I$ has the following linear resolution:

$$
(*) \cdots \stackrel{\varphi}{\rightarrow} \Lambda^{n+1} \stackrel{\varphi}{\rightarrow} \Lambda^{n+1} \stackrel{\varphi}{\rightarrow} \Lambda^{n+1} \stackrel{\varphi_{2}}{\rightarrow} \Lambda^{t} \stackrel{\varphi_{1}}{\rightarrow} \Lambda \rightarrow k \rightarrow 0
$$

where $\varphi, \varphi_{1}$, and $\varphi_{2}$ are as defined in Propositions 4.6, 4.7, 4.8.

$\underline{\text { Proof }}$ First look at $\varphi_{1} \circ \varphi_{2}=\left(\sum_{r=1}^{t} X_{r} a_{r s}\right)$.

For $1 \leq s \leq n, \sum_{r=1}^{t} X_{r} a_{r s}=\overline{X_{j_{s}}^{2}} \equiv \overline{0}$.

For $s=n+1, \sum_{r=1}^{t} X_{r} a_{r s}=X_{j_{l}}\left(c X_{j_{i}}\right)+X_{j_{k}} X_{j_{i}} \equiv \overline{0}$.

Hence, $\operatorname{Im} \varphi_{2} \subseteq \operatorname{ker} \varphi_{1}$.

Let $\left(\begin{array}{c}\overline{\gamma_{1}} \\ \vdots \\ \overline{\gamma_{t}}\end{array}\right) \in \operatorname{ker} \varphi_{1}$. Then, by Proposition 4.6,

$$
\overline{\gamma_{s}}= \begin{cases}\overline{0} & \text { if } s \in\{1, \ldots, t\} \backslash\left\{j_{1}, \ldots, j_{n}\right\} \\ \overline{X_{s} \delta_{s}} & \text { if } \left.s \in\left\{j_{1}, \ldots, j_{n}\right\} \backslash j_{k}, j_{l}\right\} \\ \overline{X_{s} \delta_{s}+X_{j_{i}} \nu} & \text { if } s=j_{k} \\ \overline{X_{s} \delta_{s}+c X_{j_{i}} \nu} & \text { if } s=j_{l}\end{cases}
$$

for some $\delta_{s}, \nu$. Then,

$$
\varphi_{2}\left(\begin{array}{c}
\overline{\delta_{j_{1}}} \\
\vdots \\
\overline{\delta_{j_{n}}} \\
\bar{\nu}
\end{array}\right)=\left(\begin{array}{c}
\overline{\gamma_{1}} \\
\vdots \\
\overline{\gamma_{t}}
\end{array}\right)
$$

So, $\operatorname{ker} \varphi_{1}=\operatorname{Im} \varphi_{2}$.

Next, $\varphi_{2} \circ \varphi=\left(a_{r s}\right)\left(b_{r s}\right)=\left(\sum_{v=1}^{n+1} a_{r v} b_{v s}\right)$.

For $r=j_{w}, w \neq l, k$ and $s=w, \sum a_{r s} b_{s w}=a_{j_{w} w} b_{w w}=\overline{X_{j_{w}}^{2}} \equiv \overline{0}$.

For $r=j_{l}$ and $s=l, \sum a_{r v} b_{v s}=a_{j_{l}} b l l=\overline{X_{j_{l}}^{2}} \equiv \overline{0}$.

For $r=j_{l}$ and $s=n+1, \sum a_{r v} b_{v s}=a_{j_{l}(n+1)} b_{(n+1)(n+1)}=\overline{c X_{j_{i}} X_{j_{i}}} \equiv \overline{0}$

For $r=j_{k}, s=k, \sum a_{r v} b_{v s}=a_{j_{k} k} b k k=\overline{X_{j_{k}}^{2}} \equiv \overline{0}$.

For $r=j_{k}, s=n+1, \sum a_{r v} b_{v s}=a_{j_{k}(n+1)} b_{(n+1)(n+1)}=\overline{X_{j_{i}} X_{j_{i}}} \equiv \overline{0}$.

For all other $r, s, \sum a_{r v} b_{v s}=0$.

So, $\operatorname{Im} \varphi \subseteq \operatorname{ker} \varphi_{2}$. 
$\operatorname{Let}\left(\begin{array}{c}\overline{X_{j_{1}} \delta_{1}} \\ \vdots \\ \frac{X_{j_{n}} \delta_{n}}{X_{j_{i}} \delta_{n+1}}\end{array}\right) \in \operatorname{ker} \varphi_{2}$.

Then,

$$
\varphi\left(\begin{array}{c}
\overline{\delta_{1}} \\
\vdots \\
\frac{\overline{\delta_{n}}}{\overline{\delta_{n+1}}}
\end{array}\right)=\left(\begin{array}{c}
\overline{X_{j_{1}} \delta_{1}} \\
\vdots \\
\overline{X_{j_{n} \delta_{n}}} \\
\overline{X_{j_{i}} \delta_{n+1}}
\end{array}\right)
$$

Hence, $\operatorname{ker} \varphi_{2}=\operatorname{Im} \varphi$.

Lastly, look at $\varphi \circ \varphi=\left(\sum_{v=1}^{n+1} b_{r v} b_{v s}\right)$.

For $r=s$ and $1 \leq r \leq n, \sum b_{r v} b_{v s}=b_{r r} b_{r r}=\overline{X_{j_{r}}^{2}} \equiv \overline{0}$.

For $r, s=n+1, \sum b_{r v} b_{v s}=b_{(n+1)(n+1)} b_{(n+1)(n+1)}=\overline{X_{j_{i}}^{2}} \equiv \overline{0}$

For all other $\mathrm{r}$ and $\mathrm{s}, \sum b_{r v} b_{v s}=0$.

Hence, $\operatorname{Im} \varphi \subseteq \operatorname{ker} \varphi$.

Let $\left(\begin{array}{c}\overline{X_{j_{1}} \delta_{1}} \\ \vdots \\ \frac{X_{j_{n}} \delta_{n}}{X_{j_{i}} \delta_{n+1}}\end{array}\right) \in \operatorname{ker} \varphi$.

Then,

$$
\varphi\left(\begin{array}{c}
\overline{\delta_{1}} \\
\vdots \\
\overline{\delta_{n}} \\
\overline{\delta_{n+1}}
\end{array}\right)=\left(\begin{array}{c}
\overline{X_{j_{1}} \delta_{1}} \\
\vdots \\
\overline{X_{j_{n}} \delta_{n}} \\
\hline X_{j_{i}} \delta_{n+1}
\end{array}\right) \in \operatorname{ker} \varphi
$$

Thus, $\operatorname{ker} \varphi=\operatorname{Im} \varphi$.

Hence, $(*)$ is exact.

For our third, and final class of Koszul algebras without a quadratic Gröbner basis, we look at the ideal with $n+z$ generators,

$$
I=\left\langle m_{1}, \ldots, m_{n}, \sum_{i=1}^{s_{1}} c_{1}^{i} p_{1}^{i}, \ldots, \sum_{i=1}^{s_{z}} c_{z}^{i} p_{z}^{i}\right\rangle
$$

as described in Lemma 4.2 with $m_{1}<\cdots<m_{c_{1}}<p_{1}^{1}<m_{c_{1}+1}<\cdots<m_{c_{2}}<p_{1}^{2}<m_{c_{2}+1}<$ $\cdots<m_{c_{z}}<p_{1}^{z}<m_{c_{z}+1}<\cdots<m_{n}$. 
We first need to understand the structure of certain ideal elements.

Proposition 4.9 Let $I=\left\langle m_{1}, \ldots, m_{n}, \sum_{j=1}^{s_{1}} c_{j}^{1} p_{j}^{1}, \ldots, \sum_{j=1}^{s_{z}} c_{j}^{z} p_{j}^{z}\right\rangle$ where $m_{i}=X_{j_{i}}^{2}$ for all $i$, and $p_{j}^{i}=X_{k_{j}^{i}}^{2}$ for all $i, j$, be an ideal of $K\left\langle X_{1}, \ldots, X_{t}\right\rangle$. Suppose that each $m_{i}$ and $p_{j}^{i}$ are distinct for all $i$. In addition, suppose each $m_{i}$ is distinct from each $p_{j}^{i}$. Then, if $X_{j_{v}} \gamma \in I$, then $\bar{\gamma} \equiv \overline{X_{j_{v}} \delta}$ for some $\delta$. Futhermore, if $X_{v} \gamma \in I$, for $v \neq j_{k}, k_{i}^{j}$ for all $i, j, k$, then $\bar{\gamma} \equiv \overline{0}$.

Proof Suppose $X_{j_{v}} \gamma \in I$. Then,

$$
X_{j_{v}} \gamma=\sum_{a=1}^{n}\left(\sum_{j=1}^{n_{a}} \mu_{j}^{a} m_{a} \rho_{j}^{a}\right)+\sum_{a=1}^{n_{\text {sum }}} \mu_{a}\left(\sum_{j=1}^{s_{a}} c_{j}^{a} p_{j}^{a}\right) \rho_{a}
$$

for some monomials $\mu_{j}^{a}, \rho_{j}^{a}, \mu_{a}, \rho_{a}$. And,

$$
\begin{gathered}
X_{j_{v}} \gamma= \\
\underbrace{\sum_{a=1}^{n}\left(\sum_{\text {subsum }} \mu_{j}^{a} m_{a} \rho_{j}^{a}\right)+\sum_{\text {subsum }} \mu_{a}\left(\sum_{j=1}^{s_{a}} c_{j}^{a} p_{j}^{a}\right) \rho_{a}}_{\text {divisible by } X_{j v}}
\end{gathered}
$$

Now, since $X_{j_{v}}$ does not divide $p_{j}^{a}$ for all $a, j$, for all a, $\mu_{a}=X_{j_{v}} \sigma_{a}$ for some monomial $\sigma_{a}$. Similarly, since $X_{j_{v}}$ does not divide $m_{a}$ for all $a \neq v$, for all $a \neq v, \mu_{j}^{a}=X_{j_{v}} \sigma_{j}^{a}$ for some monomial $\sigma_{j}^{a}$. Suppose that $\mu_{1}^{v}, \ldots, \mu_{l}^{v} \in K$, and $\mu_{l+1}^{v}, \ldots, \mu_{l_{v}}^{v} \notin K$. Then, $\mu_{l+1}^{v}=$ $X_{j_{v}} \sigma_{l+1}^{v}, \ldots, \mu_{l_{v}}^{v}=X_{j_{v}} \sigma_{l_{v}}^{v}$ for some monomials $\sigma_{l+1}^{v}, \ldots, \sigma_{l_{v}}^{v}$.

$$
\begin{gathered}
\text { Thus } X_{j_{v}} \gamma= \\
\sum_{a \neq v}\left(\sum_{\text {subsum }} X_{j_{v}} \sigma_{j}^{a} m_{a} \rho_{j}^{a}\right)+\sum_{j=1}^{l} \mu_{j}^{v} X_{j_{v}}^{2} \rho_{j}^{v}+\sum_{j=l+1}^{l_{v}} X_{j_{v}} \sigma_{j}^{v} m_{v} \rho_{j}^{v} \\
+\sum_{\text {subsum }} X_{j_{v}} \sigma_{a}\left(\sum_{j=1}^{s_{a}} c_{j}^{a} p_{j}^{a}\right) \rho_{a}
\end{gathered}
$$

Thus, $\bar{\gamma} \equiv \overline{X_{j_{v}} \delta}$ for $\delta=\sum_{1}^{l} \mu_{j}^{v} \rho_{j}^{v}$.

Now, suppose $X_{v}$ does not divide $m_{j}, p_{j}^{i}$ for all $i, j$. If

$$
X_{v} \gamma=\sum_{a=1}^{n}\left(\sum_{j=1}^{n_{a}} \mu_{j}^{a} m_{a} \rho_{j}^{a}\right)+\sum_{a=1}^{n_{\text {sum }}} \mu_{a}\left(\sum_{j=1}^{s_{a}} c_{j}^{a} p_{j}^{a}\right) \rho_{a}
$$

for some monomials $\mu_{j}^{a}, \rho_{j}^{a}, \mu_{a}, \rho_{a}$, then $\mu_{j}^{a}=X_{v} \sigma_{j}^{a}$ and $\mu_{a}=X_{v} \sigma_{a}$ for all $a, j$, since $X_{v}$ does not divide $m_{a}, p_{j}^{i}$ for all $a, i, j$. Thus, $\gamma \in I$. 
Using Proposition 4.9, we can now define all linear maps appearing in the linear resolution of the field.

Proposition 4.10 Let $\varphi_{1}: \Lambda^{t} \rightarrow \Lambda$ be defined by $\varphi_{1}=\left(X_{1} \cdots X_{t}\right)$. Then,

$$
\operatorname{ker} \varphi_{1}=\left\{\left(\begin{array}{c}
\overline{\gamma_{1}} \\
\vdots \\
\overline{\gamma_{t}}
\end{array}\right) \mid \overline{\gamma_{s}} \equiv\left\{\begin{array}{ll}
\overline{X_{s} \delta_{s}} & \text { if } s \in\left\{j_{1}, \ldots, j_{n}\right\} \\
\overline{c_{i}^{l} X_{k_{i}^{l}} \xi_{l}} & \text { if } s=k_{i}^{l} \text { for some } l \\
\overline{0} & \text { if otherwise }
\end{array}\right\}\right.
$$

for some polynomial $\delta_{s}$.

Proof Let $\left(\begin{array}{c}\overline{\gamma_{1}} \\ \vdots \\ \overline{\gamma_{t}}\end{array}\right) \in \operatorname{ker} \varphi_{1}$. Then, $\varphi_{1}\left(\begin{array}{c}\overline{\gamma_{1}} \\ \vdots \\ \overline{\gamma_{t}}\end{array}\right)=\sum_{s=1}^{t} X_{s} \overline{\gamma_{s}} \equiv \overline{0}$. So, for some monomials $\mu_{j}^{a}, \rho_{j}^{a}, \mu_{a}, \rho_{a}$,

$$
\sum_{s=1}^{t} X_{s} \gamma_{s}=\sum_{a=1}^{n}\left(\sum_{j=1}^{n_{a}} \mu_{j}^{a} m_{a} \rho_{j}^{a}\right)+\sum_{a=1}^{n_{\text {sum }}} \mu_{a}\left(\sum_{j=1}^{s_{a}} c_{j}^{a} p_{j}^{a}\right) \rho_{a}
$$

So, for $s \neq j_{v} \forall v$, and $s \neq k_{j}^{i}$ for all i,j

$$
X_{s} \gamma_{s}=\sum_{a=1}^{n}\left(\sum_{\text {subsum }} \mu_{j}^{a} m_{a} \rho_{j}^{a}\right)+\sum_{\text {subsum }} \mu_{a}\left(\sum_{j=1}^{s_{a}} c_{j}^{a} p_{j}^{a}\right) \rho_{a}
$$

for some monomials $\mu_{j}^{a}, \mu_{a}, \rho_{a}^{j}, \rho_{a}$. Hence, $X_{s} \gamma_{s} \in I$ and $\overline{\gamma_{s}} \equiv \overline{0}$. For $s=j_{v}$, (so $X_{s}^{2} \neq p_{j}^{i}$ for all $\mathrm{i}, \mathrm{j}$ )

$$
X_{j_{v}} \gamma_{j_{v}}=\sum_{a=1}^{n}\left(\sum_{\text {subsum }} \mu_{j}^{a} m_{a} \rho_{j}^{a}\right)+\sum_{\text {subsum }} \mu_{a}\left(\sum_{j=1}^{s_{a}} c_{j}^{a} p_{j}^{a}\right) \rho_{a} \in I
$$

Thus, $X_{j_{v}} \gamma_{j_{v}} \in I$ and $\overline{\gamma_{j_{v}}} \equiv \overline{X_{j_{v}} \delta_{j_{v}}}$ for some $\delta_{j_{v}}$

Lastly, if $s=k_{i}^{l}$ for some $i, l$, then for some monomials $\mu_{l}, \rho_{l}$,

$$
\text { then, } X_{s} \gamma_{s}=\sum_{a=1}^{n}\left(\sum_{\text {subsum }} \mu_{j}^{a} m_{a} \rho_{j}^{a}\right)+\sum_{\text {subsum }} \mu_{a}\left(\sum c_{j}^{a} p_{j}^{a}\right) \rho_{a}+\mu_{l} c_{i}^{l} p_{i}^{l} \rho_{l}
$$

where $\mu_{j}^{a}$ and $\mu_{a}$ are divisible by $X_{s}$.

$$
\text { Hence, } \overline{\gamma_{s}} \equiv \overline{c_{i}^{l} X_{s} \xi_{l}}
$$

for $\xi_{l}=\mu_{l} \rho_{l}$. Hence, for fixed $l, \overline{\gamma_{k_{i}^{l}}} \equiv \overline{c_{i}^{l} X_{k_{i}^{l}} \xi_{l}}$ for $\xi_{l}=\mu_{l} \rho_{l}$ for all $i=1, \ldots, s_{l}$. 
Proposition 4.11 Let $\varphi_{2}: \Lambda^{n+z} \rightarrow \Lambda^{t}$ be defined by $\varphi_{2}=\left(a_{r s}\right)$ where

$$
a_{r s}= \begin{cases}X_{r} & \text { if } 1 \leq s \leq c_{1} \text { and } r=j_{s} \\ X_{r-1} & \text { if } c_{1}+2 \leq s \leq c_{2}+1 \text { and } r=j_{s-1} \\ \vdots & \vdots \\ X_{r-z} & \text { if } c_{z}+z+1 \leq s \leq n+z \text { and } r=j_{s-z} \\ c_{j}^{v} X_{k_{j}^{v}} & \text { if } s=c_{v}+v \text { and } r=k_{j}^{v} \\ 0 & \text { if otherwise }\end{cases}
$$

(See Diagram 4.3)

Then,

$$
\operatorname{ker} \varphi_{2}=\left\{\left(\begin{array}{c}
\overline{\gamma_{1}} \\
\vdots \\
\overline{\gamma_{n+z}}
\end{array}\right) \mid \gamma_{v}=\left\{\begin{array}{ll}
\overline{X_{j_{v}} \delta_{v}} & \text { if } 1 \leq v \leq c_{1} \\
\overline{X_{j_{v-1}} \delta_{v-1}} & \text { if } c_{1}+2 \leq v \leq c_{2}+1 \\
\vdots & \vdots \\
\overline{X_{j_{v-z}} \delta_{v-z}} & \text { if } c_{z}+z+1 \leq v \leq n+z \\
\overline{0} & \text { if } v=c_{k}+k \text { for some } k
\end{array}\right\}\right.
$$

for some polynomial $\delta_{s}$.

Proof First note that we clearly have $\supseteq$. Let $\left(\begin{array}{c}\overline{\gamma_{1}} \\ \vdots \\ \overline{\gamma_{n+z}}\end{array}\right) \in \operatorname{ker} \varphi_{2}$. Then, $\varphi_{2}\left(\begin{array}{c}\overline{\gamma_{1}} \\ \vdots \\ \overline{\gamma_{n+z}}\end{array}\right)=$ $\left(\sum_{v=1}^{n+z} a_{r v} \overline{\gamma_{v}}\right) \equiv(\overline{0})$.

Thus, for $r=j_{k}$ for some $1 \leq k \leq c_{1}$, we have $\sum_{v=1}^{n+z} a_{r v} \gamma_{v}=X_{j_{k}} \gamma_{k} \in I$ and $\overline{\gamma_{k}} \equiv \overline{X_{j_{k}} \delta_{k}}$ for some $\delta_{k}$.

For $r=j_{k}$ for some $c_{1}+1 \leq k \leq c_{2}$, we have $\sum_{v=1}^{n+z} a_{r v} \gamma_{v}=X_{j_{k}} \gamma_{k+1} \in I$ and $\overline{\gamma_{k+1}} \equiv \overline{X_{j_{k}} \delta_{k}}$ for some $\delta_{k}$.

And, for $r=j_{k}$ for some $c_{z}+1 \leq k \leq n, \sum_{v=1}^{n+z} a_{r v} \gamma_{v}=X_{j_{k}} \gamma_{k} \in I$. So, $\overline{\gamma_{k+z}} \equiv \overline{X_{j_{k}} \delta_{k}}$ for some $\delta_{k}$. That is,

$$
\begin{gathered}
\text { If } 1 \leq v \leq c_{1} \text {, then } \overline{\gamma_{v}} \equiv \overline{X_{j_{v}} \delta_{v}} \\
\text { If } c_{1}+2 \leq v \leq c_{2}+1, \text { then } \overline{\gamma_{v}} \equiv \overline{X_{j_{v-1}} \delta_{v}} \\
\vdots \\
\text { If } c_{z}+z+1 \leq v \leq n+z, \text { then } \overline{\gamma_{v}} \equiv \overline{X_{j_{v-z}} \delta_{v}}
\end{gathered}
$$


for some $\delta_{v}$.

Now, fix $j$. If $r=k_{i}^{j}$ for some $i, 1 \leq i \leq s_{j}$, then $\sum a_{r v} \gamma_{v}=c_{i}^{j} X_{k_{i}^{j}} \gamma_{c_{j}+j} \in I$. Thus, for some $\delta_{j}, \overline{\gamma_{c_{j}+j}} \equiv \overline{X_{k_{i}^{j}} \delta_{j}}$ for all $1 \leq i \leq s_{j}$. Look at $X_{k_{1}^{j}} \delta_{j}-X_{k_{2}^{j}} \delta_{j}=\gamma_{c_{j}+j}-\gamma_{c_{j}+j} \in I$. Then, $X_{k_{1}^{j}} \delta_{j}-X_{k_{2}^{j}} \delta_{j}=\sum \sum \mu_{j}^{a} m_{a} \rho_{j}^{a}+\sum \mu_{a}\left(\sum C_{j}^{a} p_{j}^{a}\right) \rho_{a}$ for some monomials $\rho_{a}, \rho_{j}^{a}, \mu_{a}, \mu_{j}^{a}$.

As before, we get that $\overline{\delta_{j}} \equiv \overline{c_{i}^{j} X_{k_{i}^{j}} \xi_{j}}$ for all $i=1, \ldots, s_{j}$.

Thus, $\overline{\gamma_{c_{j}+j}} \equiv \overline{c_{i}^{j} p_{i}^{j} \xi_{j}}$ for all $i=1, \ldots s_{j}$ for some $\xi_{j}$.

Hence, $\sum_{i=1}^{s_{j}} \gamma_{c_{j}+j}=\sum_{i=1}^{s_{j}}\left(c_{i}^{j} p_{i}^{j} \xi_{j}\right)=\left(\sum_{i=1}^{s_{j}} c_{i}^{j} p_{i}^{j}\right) \xi_{j} \in I$.

Hence, $\gamma_{c_{j}+j} \in I$.

Proposition 4.12 Let $\varphi_{3}: \Lambda^{n} \rightarrow \Lambda^{n+z}$ be defined by $\varphi_{3}=\left(b_{r s}\right)$ where

$$
b_{r s}= \begin{cases}X_{j_{r}} & \text { if } 1 \leq r \leq c_{1} \text { and } r=s \\ X_{j_{r-1}} & \text { if } c_{1}+2 \leq r \leq c_{2}+1 \text { and } s=r-1 \\ \vdots & \vdots \\ X_{j_{r-z}} & \text { if } c_{z}+z+1 \leq r \leq n+z \text { and } s=r-z \\ 0 & \text { if otherwise }\end{cases}
$$

(See Diagram 4.3

Then,

$$
\operatorname{ker} \varphi_{3}=\left\{\left(\begin{array}{c}
\overline{X_{j_{1}} \delta_{1}} \\
\vdots \\
\overline{X_{j_{n}} \delta_{n}}
\end{array}\right) \mid \delta_{k} \in K\left\langle X_{1}, \ldots, X_{t}\right\rangle \forall k\right\}
$$

Proof First note that we clearly have $\supseteq$. Let $\left(\begin{array}{c}\overline{\gamma_{1}} \\ \vdots \\ \overline{\gamma_{n}}\end{array}\right) \in \operatorname{ker} \varphi_{3}$. Then, $\varphi_{3}\left(\begin{array}{c}\overline{\gamma_{1}} \\ \vdots \\ \overline{\gamma_{n}}\end{array}\right)=$ $\left(\sum_{v=1}^{n} b_{r v} \overline{\gamma_{v}}\right) \equiv(\overline{0})$. Thus, for $1 \leq r \leq c_{1}$, we have $\sum_{v=1}^{n} b_{r v} \gamma_{v}=X_{j_{r}} \gamma_{r} \in I$ and $\overline{\gamma_{r}} \equiv \overline{X_{j_{r}} \delta_{r}}$ for some $\delta_{r}$. And, for $c_{z}+z+1 \leq r \leq n+z, \sum_{v=1}^{n} b_{r v} \gamma_{v}=X_{j_{r-z}} \gamma_{r-z} \in I$. So, $\overline{\gamma_{r-z}} \equiv \overline{X_{j_{r-z}} \delta_{r-z}}$ for some $\delta_{r-z}$. And, in general, for $c_{k}+k+1 \leq r \leq c_{k+1}+k, \sum_{v=1}^{n} b_{r v} \gamma_{v}=X_{j_{r-k}} \gamma_{r-k} \in I$ and $\overline{\gamma_{r-k}} \equiv \overline{X_{j_{r-k}} \delta_{r-k}}$ for some $\delta_{r-k}$, for $1 \leq k \leq z-1$.

Thus, for all $r, \overline{\gamma_{r}} \equiv \overline{X_{j_{r}} \delta_{r}}$ for some $\delta_{r}$.

Proposition 4.13 Let $\varphi: \Lambda^{n} \rightarrow \Lambda^{n}$ be defined by $\varphi=\left(c_{r s}\right)$ where

$$
c_{r s}= \begin{cases}X_{j_{r}} & \text { if } r=s \\ 0 & \text { if otherwise }\end{cases}
$$


Then,

$$
\operatorname{ker} \varphi=\left\{\left(\begin{array}{c}
\overline{X_{j_{1}} \delta_{1}} \\
\vdots \\
\overline{X_{j_{n}} \delta_{n}}
\end{array}\right) \mid \delta_{k}\right\}
$$

Proof First note that we clearly have $\supseteq$. Let $\left(\begin{array}{c}\overline{\gamma_{1}} \\ \vdots \\ \overline{\gamma_{n}}\end{array}\right) \in \operatorname{ker} \varphi$. Then, $\varphi\left(\begin{array}{c}\overline{\gamma_{1}} \\ \vdots \\ \overline{\gamma_{n}}\end{array}\right)=$ $\left(\sum_{v=1}^{n} c_{r v} \overline{\gamma_{v}}\right) \equiv(\overline{0})$. Thus, for $1 \leq r \leq n$, we have $\sum_{v=1}^{n} b_{r v} \gamma_{v}=X_{j_{r}} \gamma_{r} \in I$ and $\overline{\gamma_{r}} \equiv \overline{X_{j_{r}} \delta_{r}}$ for some $\delta_{r}$.

Theorem 4.3 Let $I=\left\langle m_{1}, \ldots, m_{n}, \sum_{j=1}^{s_{1}} c_{j}^{1} p_{j}^{1}, \ldots, \sum_{j=1}^{s_{z}} c_{j}^{z} p_{j}^{z}\right\rangle$ be an ideal in $K\left\langle X_{1}, \ldots, X_{t}\right\rangle$ where $m_{i}=X_{j_{i}}^{2}, c_{j}^{i} \in K$, and $p_{j}^{i}=X_{k_{j}^{i}}^{2}$ for all $i, j$. Suppose in addition that $m_{1}<m_{2}<\ldots<$ $m_{c_{1}}<p_{1}^{1}=\operatorname{tip}\left(\sum c_{j}^{1}\right)<m_{c_{1}+1}<\ldots<m_{c_{2}}<p_{1}^{2}=\operatorname{tip}\left(\sum c_{j}^{2} p_{j}^{2}\right)<m_{c_{2}+1}<\ldots<m_{c_{z}}<p_{1}^{z}=$ $\operatorname{tip}\left(\sum c_{j}^{z} p_{j}^{z}\right)<m_{c_{z}+1}<\ldots<m_{n}$ with each $m_{i}$ distinct, each $m_{i}$ distinct from the $p_{j}^{i}$ 's and each $p_{j}^{i}$ distinct. Then, $\Lambda=K\left\langle X_{1}, \ldots, X_{t}\right\rangle / I$ has the following linear resolution:

$$
(*) \cdots \stackrel{\varphi}{\rightarrow} \Lambda^{n} \stackrel{\varphi}{\rightarrow} \Lambda^{n} \stackrel{\varphi_{3}}{\rightarrow} \Lambda^{n+z} \stackrel{\varphi_{2}}{\rightarrow} \Lambda^{t} \stackrel{\varphi_{7}}{\rightarrow} \Lambda \rightarrow k \rightarrow 0
$$

where $\varphi, \varphi_{1}, \varphi_{2}$, and $\varphi_{3}$ are as defined in Propositions 4.10, 4.11, 4.12, 4.13.

$\underline{\text { Proof }}$ First look at $\varphi_{1} \circ \varphi_{2}=\left(\sum_{r=1}^{t} X_{r} a_{r s}\right)$.

For $1 \leq s \leq c_{1}, \sum_{r=1}^{t} X_{r} a_{r s}=\overline{X_{j_{s}}^{2}} \equiv \overline{0}$

For $s=c_{1}+1, \sum_{r=1}^{t} X_{r} a_{r s}=\sum_{j=1}^{s_{1}} c_{j}^{1} \overline{X_{k_{j}^{1}}^{2}} \equiv \overline{0}$

For $c_{1}+2 \leq s \leq c_{2}+1, \sum_{r=1}^{t} X_{r} a_{r s}=\overline{X_{j_{s-1}}^{2}} \equiv \overline{0}$

For $s=c_{2}+2, \sum_{r=1}^{t} X_{r} a_{r s}=\sum_{j=1}^{s_{2}} c_{j}^{2} \overline{X_{k_{j}^{2}}^{2}} \equiv \overline{0}$

For $s=c_{z}+z, \sum_{r=1}^{t} X_{r} a_{r s}=\sum_{j=1}^{s_{z}} c_{j}^{z} \overline{X_{k_{j}^{z}}^{2}} \equiv \overline{0}$

Hence, $\varphi_{1} \circ \varphi_{2}=(0)_{1 \times(n)}$. Thus, $\operatorname{Im} \varphi_{2} \subseteq \operatorname{ker} \varphi_{1}$. 
Let $\left(\begin{array}{c}\overline{\gamma_{1}} \\ \vdots \\ \overline{\gamma_{t}}\end{array}\right) \in \operatorname{ker} \varphi_{1}$. Then, by Proposition 4.10,

$$
\overline{\gamma_{s}} \equiv \begin{cases}\overline{X_{s} \delta_{s}} & \text { if } s \in\left\{j_{1}, \ldots, j_{n}\right\} \\ \overline{c_{i}^{j} X_{k_{i}^{j}} \xi_{j}} & \text { if } s=k_{i}^{j} \text { for some } i, j \\ \overline{0} & \text { if otherwise }\end{cases}
$$

for some $\delta_{s}$.

Then,

$$
\left(\begin{array}{c}
\overline{\delta_{j_{1}}} \\
\vdots \\
\overline{\delta_{j_{1}}} \\
\frac{\overline{\xi_{1}}}{\delta_{j_{c_{1}+1}}} \\
\vdots \\
\frac{\delta_{j_{c_{2}}}}{\overline{\xi_{2}}} \\
\frac{\delta_{j_{c_{2}+1}}}{\vdots} \\
\frac{\delta_{j_{c_{z}}}}{\overline{\xi_{z}}} \\
\frac{\delta_{j_{c_{z}+1}}}{\vdots} \\
\frac{\delta_{j_{n}}}{\overline{\delta_{t}}}
\end{array}\right)=\left(\begin{array}{c}
\overline{\gamma_{1}} \\
\vdots \\
\overline{\gamma_{t}}
\end{array}\right)
$$

Hence, $\operatorname{ker} \varphi_{1} \subseteq \operatorname{im} \varphi_{2}$. So, $\operatorname{ker} \varphi_{1}=\operatorname{Im} \varphi_{2}$.

Next, $\varphi_{2} \circ \varphi_{3}=\left(\sum_{v=1}^{n+z} a_{r v} b_{v s}\right)$.

Now, for $r=j_{w}$ for some $1 \leq w \leq n \sum_{v=1}^{n+z} a_{r v} b_{v s}=a_{j_{w} w} b_{w w}=\overline{X_{j_{w}}^{2}} \equiv \overline{0}$

And, for all other $\mathrm{r}, \sum_{v=1}^{n+z} a_{r v} b_{v s}=0$. Hence, $\operatorname{im} \varphi_{3} \subseteq \operatorname{ker} \varphi_{2}$. 


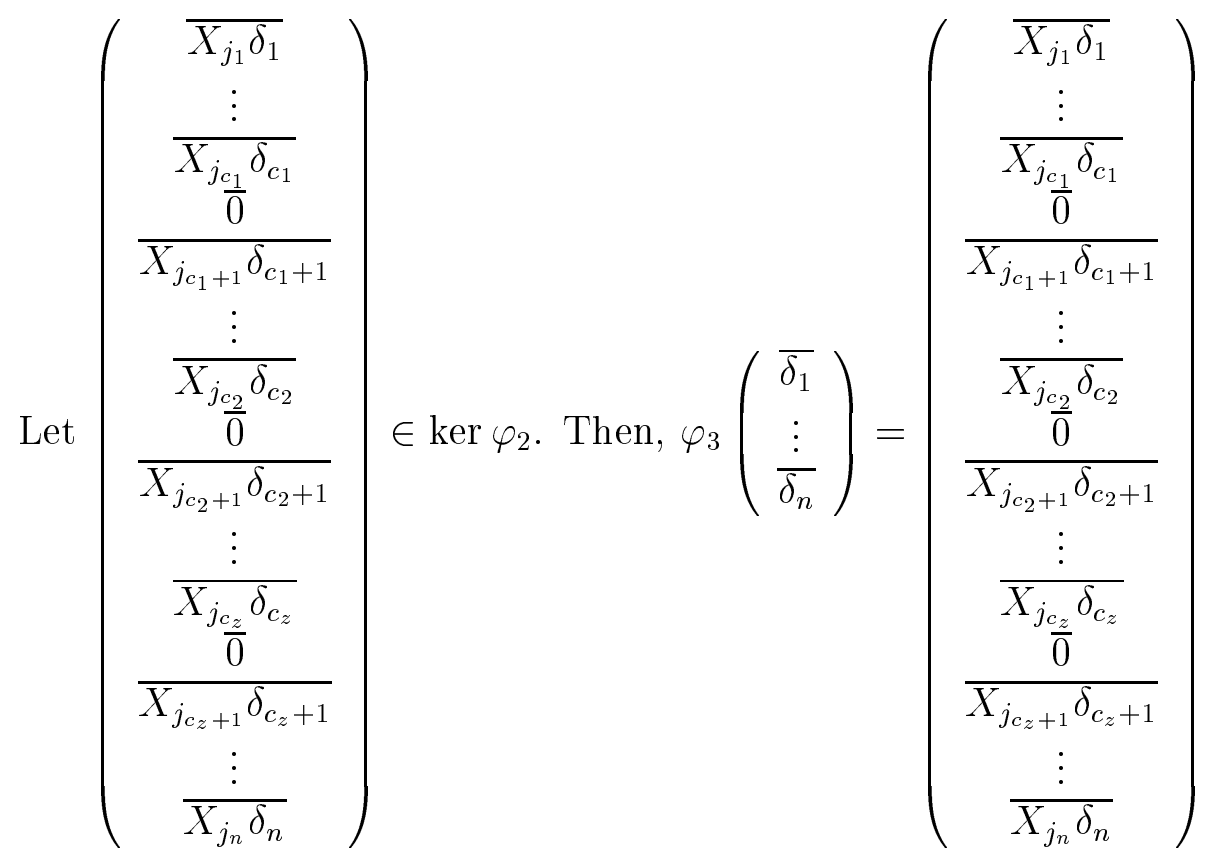

Hence, $\operatorname{ker} \varphi_{2}=\operatorname{Im} \varphi$.

$$
\varphi_{3} \circ \varphi=\left(\sum_{v=1}^{n} b_{r v} c_{v s}\right)
$$

Now, for $r \neq c_{i}+i, 1 \leq i \leq z, s=r$ for $1 \leq r \leq c_{1}$ and $s=r-i$ for $c_{i}+1 \leq r \leq c_{i+1}+i$, $\sum_{v=1}^{n} b_{r v} c_{v s}=\overline{X_{j_{r}}^{2}} \equiv \overline{0}$.

And, for all other r, $\sum_{v=1}^{n} b_{r v} c_{v s}=0$. Hence, $\varphi_{3} \circ \varphi \equiv(0)_{(n+z) \times(n)}$ Thus, $\operatorname{Im} \varphi \subseteq \operatorname{ker} \varphi_{3}$.

Let $\left(\begin{array}{c}\overline{X_{j_{1} \delta_{1}}} \\ \vdots \\ \overline{X_{j_{n}} \delta_{n}}\end{array}\right) \in \operatorname{ker} \varphi_{3}$. Then, $\varphi\left(\begin{array}{c}\overline{\delta_{1}} \\ \vdots \\ \overline{\delta_{n}}\end{array}\right)=\left(\begin{array}{c}\overline{X_{j_{1} \delta_{1}}} \\ \vdots \\ \overline{X_{j_{n}} \delta_{n}}\end{array}\right)$. Thus, $\operatorname{ker} \varphi_{3}=\operatorname{Im} \varphi$.

And, finally, $\varphi \circ \varphi=\left(\sum_{v=1}^{n} c_{r v} c_{v s}\right)$

where for $1 \leq r \leq n$ and $r=s, \sum_{v=1}^{n} c_{r v} c_{v s}=\overline{X_{j_{r}}^{2}} \equiv \overline{0}$. For all other $\mathrm{r}$ and $\mathrm{s}, \sum_{v=1}^{n} c_{r v} c_{v s}=0$.

Hence, $\operatorname{im} \varphi \subseteq \operatorname{ker} \varphi$. Now, let $\left(\begin{array}{c}\overline{X_{j_{1} \delta_{1}}} \\ \vdots \\ \overline{X_{j_{n}} \delta_{n}}\end{array}\right) \in \operatorname{ker} \varphi$. Then, $\varphi\left(\begin{array}{c}\overline{\delta_{1}} \\ \vdots \\ \overline{\delta_{n}}\end{array}\right)=\left(\begin{array}{c}\overline{X_{j_{1}} \delta_{1}} \\ \vdots \\ \overline{X_{j_{n}} \delta_{n}}\end{array}\right)$. So, $\operatorname{im} \varphi=\operatorname{ker} \varphi$. Hence, $(*)$ is exact. 


\section{Chapter 5}

\section{A Non-Koszul Algebra}

We now arrive at the last of our investigated classes of quotients of the non-commutative polynomial ring. Throughout this chapter, $m_{1}=X_{j_{1}}^{2}, \ldots, m_{n}=X_{j_{n}}^{2}$ are perfect square monomials in $K\left\langle X_{1}, \ldots, X_{t}\right\rangle$ and $\Lambda=K\left\langle X_{1}, \ldots, X_{t}\right\rangle / I$. Additionally, by $\bar{\gamma}$ we mean $\gamma+I$.

Lemma 5.1 If $p+c q=X_{j_{i}} X_{\beta}+c X_{\xi} X_{j_{i}}$ for some $1 \leq i \leq n$ and some $\beta \neq \xi, \beta, \xi \neq j_{i}$, then $I=\left\langle m_{1}, \ldots, m_{n}, p+c q\right\rangle$ has no quadratic Gröbner basis.

Proof Let us suppose tip $(p+c q)=X_{j_{i}} X_{\beta}\left(\operatorname{tip}(p+c q)=X_{\xi} X_{j_{i}}\right.$ is analogous $)$ and consider

$$
o\left(p+c q, m_{i}\right)=X_{j_{i}}\left(X_{j_{i}} X_{\beta}+c X_{\xi} X_{j_{i}}\right)-\left(X_{j_{i}}^{2}\right) X_{\beta}=c X_{j_{i}} X_{\xi} X_{j_{i}}
$$

Now, since $X_{\xi} \neq X_{j_{i}}$ or $X_{\beta}, X_{j_{i}} X_{\xi} X_{j_{i}}$ is irreducible. Thus, we have an irreducible cubic term to add to the Gröbner basis for I.

That is, if neither $p$ nor $q$ is a perfect square, $\operatorname{supp}(p) \neq \operatorname{supp}(q)$, and if for some $i$, $\operatorname{supp}(p) \cap \operatorname{supp}(q) \cap \operatorname{supp}\left(m_{i}\right) \neq \phi$ and $m_{i} \mid p q$ or $q p$, then $I=\left\langle m_{1}, \ldots, m_{n}, p+c q\right\rangle$ has no quadratic Gröbner basis.

As repeatedly emphasized, the existence of a quadratic Gröbner basis is not necessary for the resulting algebra to be Koszul. In fact, in this case, the resulting algebra is not Koszul. To show this, we will describe the maps up to the first non-linear map of the field resolution, as well as provide a description of that first non-linear map. We begin with a result concerning ideal membership.

Proposition 5.1 Let $I=\left\langle m_{1}, \ldots, m_{n}, p+c q\right\rangle$ with $m_{s}=X_{j_{s}}^{2}$ for all $s$, and $p+c q=$ $X_{j_{i}} X_{\beta}+c X_{\xi} X_{j_{i}}$ for some $1 \leq i \leq n, \beta \neq \xi$ and $j_{i} \neq \beta, \xi$. If $X_{j_{v}} \gamma \in I$ for some $v \neq i$ and $j_{v} \neq \xi$, then $\bar{\gamma} \equiv \overline{X_{j_{v}} \delta}$ for some $\delta$. Futhermore, if $X_{v} \gamma \in I$ for $v \neq j_{s}$ for all $s$ and $v \neq \beta, \xi$, then $\gamma \in I$. 
Proof Let $X_{j_{v}} \gamma \in I$. Then, for some monomials $\rho_{j}^{a}, \rho_{j}, \mu_{j}^{a}, \mu_{j}$,

$$
X_{j_{v}} \gamma=\sum_{a=1}^{n}\left(\sum_{j=1}^{n_{a}} \mu_{j}^{a} m_{a} \rho_{j}^{a}\right)+\sum_{j=1}^{n_{s u m}} \mu_{j}\left(X_{j_{i}} X_{\beta}+c X_{\xi} X_{j_{i}}\right) \rho_{j}
$$

Since $j_{v} \neq \xi, j_{i}, \mu_{j} \notin K \backslash 0$ for all $1 \leq j \leq n_{\text {sum }}$. Hence, for all $j, \mu_{j}=X_{j_{v}} \sigma_{j}$ for some monomial $\sigma_{j}$. Similarly, for all $j$ and all $a \neq v, \mu_{j}^{a}=X_{j_{v}} \sigma_{j}^{a}$ for some monomials $\sigma_{j}^{a}$.

$$
\text { Thus, } X_{j_{v}} \gamma=\sum_{a \neq v}\left(\sum^{n_{a}} X_{j_{v}} \sigma_{j}^{a} m_{a} \rho_{j}^{a}\right)+\sum^{n_{v}} \mu_{j}^{v} m_{v} \rho_{j}^{v}+\sum^{n_{\text {sum }}} X_{j_{v}} \sigma_{j}\left(X_{j_{i}} X_{\beta}+c X_{\xi} X_{j_{i}}\right) \rho_{j}
$$

Now, suppose $\mu_{1}^{v}, \ldots, \mu_{\lambda_{v}}^{v} \in K \backslash 0$ and $\mu_{\lambda_{v}+1}^{v}, \ldots, \mu_{n_{v}}^{v} \notin K \backslash 0$. Then, for all $\lambda_{v}+1 \leq s \leq n_{v}$, $\mu_{s}^{v}=X_{j_{v}} \sigma_{s}^{v}$ for some $\sigma_{s}^{v}$.

$$
\begin{gathered}
\text { Hence } X_{j_{v}} \gamma=\sum_{a \neq v}\left(\sum^{n_{a}} X_{j_{v}} \sigma_{j}^{a} m_{a} \rho_{j}^{a}\right)+\sum^{\lambda_{v}} \mu_{j}^{v} X_{j_{v}}^{2} \rho_{j}^{v}+ \\
\sum_{\lambda_{v}+1}^{n_{v}} X_{j_{v}} \sigma_{j}^{v} m_{v} \rho_{j}^{v}+\sum^{n_{s u m}} X_{j_{v}} \sigma_{j}\left(X_{j_{i}} X_{\beta}+c X_{\xi} X_{j_{i}}\right) \rho_{j}
\end{gathered}
$$

Hence, $\bar{\gamma} \equiv \overline{X_{j_{v}} \delta}$ for some $\delta$. Futhermore, if for some monomials $\mu_{j}, \rho_{j}, \mu_{j}^{a}, \rho_{j}^{a}$,

$$
X_{v} \gamma=\sum_{a=1}^{n}\left(\sum_{j=1}^{n_{a}} \mu_{j}^{a} m_{a} \rho_{j}^{a}\right)+\sum_{j=1}^{n_{\text {sum }}} \mu_{j}\left(X_{j_{i}} X_{\beta}+c X_{\xi} X_{j_{i}}\right) \rho_{j}
$$

where $X_{v}$ does not appear as any $m_{a}$ nor as $X_{\beta}$ or $X_{\xi}$, then we must have $X_{v} \mid \mu_{j}$ and $\mu_{j}^{a}$ for all $a, j$. Hence, $\bar{\gamma} \equiv \overline{0}$.

We will show that $\Lambda$, for $I$ defined in Proposition 5.1, is not Koszul. Thus, we will show that regardless of order, the field has no linear resolution. For Propositions 5.2, 5.3, we suppose tip $(p+c q)=X_{j_{i}} X_{\beta}$. The next result gives a description of the kernel of the last linear map in the resolution of the field supposing that tip $(p+c q)=X_{j_{i}} X_{\beta}$. This leads to Proposition 5.3, showing the resolution is exact at the point of non-linearity.

Proposition 5.2 Let $\varphi_{2}: \Lambda^{n+1} \longrightarrow \Lambda^{t}$ be defined by $\varphi_{2}=\left(a_{r s}\right)$ where

$$
a_{r s}= \begin{cases}X_{j_{s}} & \text { if } 1 \leq s \leq i, r=j_{s} \\ X_{\beta} & \text { if } s=i+1, r=j_{i} \\ c X_{j_{i}} & \text { if } s=i+1, r=\xi \\ X_{j_{s-1}} & \text { if } i+2 \leq s \leq n+1, r=j_{s-1} \\ 0 & \text { if otherwise }\end{cases}
$$


(See Diagram 5.1, Case I)

Then,

$$
\operatorname{ker} \varphi_{2}=\left\{\left(\begin{array}{c}
\overline{X_{j_{1}} \delta_{1}} \\
\frac{\vdots}{X_{j_{k-1} \delta_{k-1}}} \\
\frac{X_{j_{k+1}} \delta_{k+1}}{\vdots} \\
\frac{X_{j_{i-1} \delta_{i-1}}}{X_{j_{i}} \delta_{i}+X_{\beta} X_{j_{i}} \theta} \\
\frac{\bar{\nabla}_{i+1}}{X_{j_{i+1}} \delta_{i+1}} \\
\frac{\vdots}{X_{j_{n}} \delta_{n}}
\end{array}\right) \mid \delta_{1}, \ldots, \delta_{n+1}, \theta \in K\left\langle X_{1}, \ldots, X_{t}\right\rangle\right\}
$$

where

$$
\nabla_{k}= \begin{cases}X_{j_{k}} \delta_{k} & \text { if } X_{\xi}=X_{j_{k}} \text { for some } k, X_{\beta} \neq X_{j_{l}} \text { for all } l . \\ X_{j_{k}} \delta_{k} & \text { if } X_{\beta}=X_{j_{l}} \text { for some } l, X_{\xi} \neq X_{j_{k}} \text { for all } k . \\ X_{j_{k}} \delta_{k}+c X_{j_{i}} \mu & \text { if } X_{\xi}=X_{j_{k}} \text { for some } k, X_{\beta}=X_{j_{l}} \text { for some } l .\end{cases}
$$

and

$$
\nabla_{i+1}= \begin{cases}0 & \text { if } X_{\xi}=X_{j_{k}} \text { for some } k, X_{\beta} \neq X_{j_{l}} \text { for all } l . \\ X_{j_{l}} X_{j_{i}} \mu & \text { if } X_{\beta}=X_{j_{l}} \text { for some } l, X_{\xi} \neq X_{j_{k}} \text { for all } k \\ c^{-1} X_{j_{l}} \mu^{\prime} & \text { if } X_{\xi}=X_{j_{k}} \text { for some } k, X_{\beta}=X_{j_{l}} \text { for some } l .\end{cases}
$$

$\underline{\text { Proof }} \underline{\text { Case } 1} \operatorname{Suppose} X_{\xi}=X_{j_{k}}$ for some $k, X_{\beta} \neq X_{j_{l}}$ for all $l$. Let $\left(\begin{array}{c}\overline{\gamma_{1}} \\ \vdots \\ \overline{\gamma_{n+1}}\end{array}\right) \in \operatorname{ker} \varphi_{2}$. Then $\varphi_{2}\left(\begin{array}{c}\overline{\gamma_{1}} \\ \vdots \\ \overline{\gamma_{n+1}}\end{array}\right)=\left(\begin{array}{c}\sum a_{1 s} \overline{\gamma_{s}} \\ \vdots \\ \sum a_{t s} \overline{\gamma_{s}}\end{array}\right) \equiv\left(\begin{array}{c}\overline{0} \\ \vdots \\ \overline{0}\end{array}\right)$. That is, for all $1 \leq r \leq t, \sum a_{r s} \gamma_{s} \in I$.

For $r=j_{v}, 1 \leq v \leq k-1$ and $k+1 \leq v \leq i-1, \sum a_{j_{v} s} \gamma_{s}=X_{j_{v}} \gamma_{v} \in I$ and $\overline{\gamma_{v}} \equiv \overline{X_{j_{v}} \delta_{v}}$ for some $\delta_{v}$ by Proposition 5.1.

For $r=j_{v}, i+1 \leq v \leq n, \sum a_{j_{v}} \gamma_{s}=X_{j_{v}} \gamma_{v+1} \in I$ and $\overline{\gamma_{v+1}} \equiv \overline{X_{j_{v}} \delta_{v+1}}$ for some $\delta_{v+1}$ by Proposition 5.1. 
For $r=j_{k}, \sum a_{j_{k} s} \gamma_{s}=X_{j_{k}} \gamma_{k}+c X_{j_{i}} \gamma_{i+1} \in I$. Thus, $X_{j_{k}} \gamma_{k}+c X_{j_{i}} \gamma_{i+1}=\sum_{a=1}^{n} \sum_{j=1}^{n_{a}} \mu_{j}^{a} m_{a} \rho_{j}^{a}+$ $\sum_{j=1}^{n_{s u m}} \mu_{j}\left(X_{j_{i}} X_{\beta}+c X_{\xi} X_{j_{i}}\right) \rho_{j}$ for some monomials $\mu_{j}, \mu_{j}^{a}, \rho_{j}, \rho_{j}^{a}$. Hence,

$$
X_{j_{k}} \gamma_{k}=\sum_{a=1}^{n} \sum_{\substack{\text { subsum } \\
\text { over j }}} \mu_{j}^{a} m_{a} \rho_{j}^{a}+\sum_{\begin{array}{c}
\text { subsum over j } \\
\text { such that } X_{j_{k}} \mid \mu_{j}
\end{array}} \mu_{j}\left(X_{j_{i}} X_{\beta}+c X_{\xi} X_{j_{i}}\right) \rho_{j}+
$$

$$
\sum_{\substack{\text { subsum over } \mathrm{j} \\ \text { such that } \mu_{j} \in K \backslash 0}} \mu_{j}\left(c X_{j_{k}} X_{j_{i}}\right) \rho_{j}
$$

and

$$
\begin{gathered}
X_{j_{i}} \gamma_{i+1}=\sum_{a=1}^{n} \sum_{\begin{array}{c}
\text { subsum } \\
\text { over j }
\end{array}} \mu_{j}^{a} m_{a} \rho_{j}^{a}+\sum_{\begin{array}{c}
\text { subsum over j } \\
\text { such that } X_{j_{i}} \mid \mu_{j}
\end{array}} \mu_{j}\left(X_{j_{i}} X_{\beta}+c X_{\xi} X_{j_{i}}\right) \rho_{j}+ \\
\sum_{\begin{array}{c}
\text { subsum over j } \\
\text { such that } \mu_{j} \in K \backslash 0
\end{array}} \mu_{j}\left(X_{j_{i}} X_{\beta}\right) \rho_{j}
\end{gathered}
$$

Thus, $\overline{\gamma_{k}} \equiv \overline{X_{j_{k}} \delta_{k}+c X_{j_{i}} \mu}$ and $\overline{\gamma_{i+1}} \equiv \overline{X_{j_{i}} \delta_{i+1}+X_{\beta} \mu}$ for some $\delta_{k}, \delta_{i+1}, \mu$.

Lastly, for $r=j_{i}, \sum a_{j_{i}} \gamma_{s}=X_{j_{i}} \gamma_{i}+X_{\beta} \gamma_{i+1} \in I$.

Thus, $X_{j_{i}} \gamma_{i}+X_{\beta} \gamma_{i+1}=\sum_{a=1}^{n} \sum_{j=1}^{n_{a}} \mu_{j}^{a} m_{a} \rho_{j}^{a}+\sum_{j=1}^{n_{s u m}} \mu_{j}\left(X_{j_{i}} X_{\beta}+c X_{\xi} X_{j_{i}}\right) \rho_{j}$ for some monomials $\mu_{j}, \mu_{j}^{a}, \rho_{j}, \rho_{j}^{a}$.

$$
\begin{gathered}
\text { Hence, } X_{j_{i}} \gamma_{i}=\sum_{a=1}^{n} \sum_{\substack{\text { subsum } \\
\text { over j }}} \mu_{j}^{a} m_{a} \rho_{j}^{a}+ \\
\sum_{\substack{\text { subsum over j } \\
\text { such that } X_{j_{i}} \mid \mu_{j}}} \mu_{j}\left(X_{j_{i}} X_{\beta}+c X_{\xi} X_{j_{i}}\right) \rho_{j}+\sum_{\mathcal{I}} \mu_{j}\left(X_{j_{i}} X_{\beta}\right) \rho_{j}
\end{gathered}
$$

where $\mathcal{I}=\left\{j \mid \mu_{j} \in K \backslash 0\right.$ and $\mu_{j} c X_{j_{k}} X_{j_{i}} \rho_{j}$ cancels with a $\mu_{j^{\prime}}^{a^{\prime}} m_{a^{\prime}} \rho_{j^{\prime}}^{a^{\prime}}$ term $\}$

And,

$$
X_{\beta} \gamma_{i+1}=\sum_{a=1}^{n} \sum_{\substack{\text { subsum } \\
\text { over j }}} \mu_{j}^{a} m_{a} \rho_{j}^{a}+\sum_{\begin{array}{c}
\text { subsum over j } \\
\text { such that } X_{\beta} \mid \mu_{j}
\end{array}} \mu_{j}\left(X_{j_{i}} X_{\beta}+c X_{\xi} X_{j_{i}}\right) \rho_{j}
$$

Thus, $\overline{\gamma_{i}} \equiv \overline{X_{j_{i}} \delta_{i}+X_{\beta} X_{j_{i}} \theta}$ for some $\delta_{i}, \theta$ and $\overline{\gamma_{i+1}} \equiv \overline{0}$. 
So, we have two representations for $\gamma_{i+1}: \overline{0} \equiv \overline{\gamma_{i+1}} \equiv \overline{X_{j_{i}} \delta_{i+1}+X_{\beta} \mu}$. Thus, $X_{j_{i}} \delta_{i+1}+X_{\beta} \mu \in I$ As above, we get $\bar{\mu} \equiv \overline{0}$. Hence, $\overline{\gamma_{k}} \equiv \overline{X_{j_{k}} \delta_{k}}$ for some $\delta_{k}$.

$\underline{\text { Case } 2}$ Suppose $X_{\beta}=X_{j_{l}}$ for some $l, X_{\xi} \neq X_{j_{k}}$ for all $k$. Let $\left(\begin{array}{c}\overline{\gamma_{1}} \\ \vdots \\ \overline{\gamma_{n+1}}\end{array}\right) \in \operatorname{ker} \varphi_{2}$. Then $\varphi_{2}\left(\begin{array}{c}\overline{\gamma_{1}} \\ \vdots \\ \overline{\gamma_{n+1}}\end{array}\right)=\left(\begin{array}{c}\sum a_{1 s} \overline{\gamma_{s}} \\ \vdots \\ \sum a_{t s} \overline{\gamma_{s}}\end{array}\right) \equiv\left(\begin{array}{c}\overline{0} \\ \vdots \\ \overline{0}\end{array}\right)$. That is, for all $1 \leq r \leq t, \sum a_{r s} \gamma_{s} \in I$.

For $r=j_{v}, 1 \leq v \leq i-1, \sum a_{j_{v} s} \gamma_{s}=X_{j_{v}} \gamma_{v} \in I$ and $\overline{\gamma_{v}} \equiv \overline{X_{j_{v}} \delta_{v}}$ for some $\delta_{v}$ by Proposition 5.1 .

For $r=j_{v}, i+1 \leq v \leq n, \sum a_{j_{v} s} \gamma_{s}=X_{j_{v}} \gamma_{v+1} \in I$ and $\overline{\gamma_{v+1}} \equiv \overline{X_{j_{v}} \delta_{v+1}}$ for some $\delta_{v+1}$ by Proposition 5.1.

For $r=\xi, \sum a_{\xi s} \gamma_{s}=c X_{j_{i}} \gamma_{i+1} \in I$. Thus, $c X_{j_{i}} \gamma_{i+1}=\sum_{a=1}^{n} \sum_{j=1}^{n_{a}} \mu_{j}^{a} m_{a} \rho_{j}^{a}+\sum_{j=1}^{n_{s u m}} \mu_{j}\left(X_{j_{i}} X_{\beta}+\right.$ $\left.c X_{\xi} X_{j_{i}}\right) \rho_{j}$ for some monomials $\mu_{j}, \mu_{j}^{a}, \rho_{j}, \rho_{j}^{a}$.

Hence,

$$
\begin{gathered}
X_{j_{i}} \gamma_{i+1}=\sum_{a=1}^{n} \sum_{\substack{\text { subsum } \\
\text { over j }}} \mu_{j}^{a} m_{a} \rho_{j}^{a}+ \\
\sum_{\substack{\text { subsum over j } \\
\text { such that } X_{j_{i}} \mid \mu_{j}}} \mu_{j}\left(X_{j_{i}} X_{\beta}+c X_{\xi} X_{j_{i}}\right) \rho_{j}+\sum_{\mathcal{I}} \mu_{j}\left(X_{j_{i}} X_{\beta}\right) \rho_{j}
\end{gathered}
$$

where $\mathcal{I}=\left\{j \mid \mu_{j} \in K \backslash 0\right.$ and $\mu_{j} X_{\xi} X_{j_{i}} \rho_{j}$ cancels with a $\mu_{j^{\prime}}^{a^{\prime}} \rho_{j^{\prime}}^{\left.a^{\prime} \text { term }\right\}}$

So, $\overline{\gamma_{i+1}} \equiv \overline{X_{j_{i}} \delta_{i+1}+X_{j_{l}} X_{j_{i}} \mu}$ for some $\delta_{i+1}, \mu$.

Lastly, for $r=j_{i}, \sum a_{j_{i} s} \gamma_{s}=X_{j_{i}} \gamma_{i}+X_{j_{l}} \gamma_{i+1} \in I$.

Thus, $X_{j_{i}} \gamma_{i}+X_{j_{l}} \gamma_{i+1}=\sum_{a=1}^{n} \sum_{j=1}^{n_{a}} \mu_{j}^{a} m_{a} \rho_{j}^{a}+\sum_{j=1}^{n_{s u m}} \mu_{j}\left(X_{j_{i}} X_{\beta}+c X_{\xi} X_{j_{i}}\right) \rho_{j}$ for some monomials $\mu_{j}, \mu_{j}^{a}, \rho_{j}, \rho_{j}^{a}$.

$$
\begin{gathered}
\text { Thus, } X_{j_{i}} \gamma_{i}=\sum_{a=1}^{n} \sum_{\substack{\text { subsum } \\
\text { over j }}} \mu_{j}^{a} m_{a} \rho_{j}^{a}+ \\
\sum_{\substack{\text { subsum over j } \\
\text { such that } X_{j_{i}} \mid \mu_{j}}} \mu_{j}\left(X_{j_{i}} X_{\beta}+c X_{\xi} X_{j_{i}}\right) \rho_{j}+\sum_{\mathcal{I}} \mu_{j}\left(X_{j_{i}} X_{\beta}\right) \rho_{j}
\end{gathered}
$$


where $\mathcal{I}=\left\{j \mid \mu_{j} \in K \backslash 0\right.$ and $\mu_{j} c X_{\xi} X_{j_{i}} \rho_{j}$ cancels with a $\mu_{j^{\prime}}^{a^{\prime}} m_{a^{\prime}} \rho_{j^{\prime}}^{a^{\prime}}$ term $\}$

And,

$$
X_{j_{l}} \gamma_{i+1}=\sum_{a=1}^{n} \sum_{\substack{\text { subsum } \\
\text { over j }}} \mu_{j}^{a} m_{a} \rho_{j}^{a}+\sum_{\begin{array}{c}
\text { subsum over j } \\
\text { such that } X_{j_{l}} \mid \mu_{j}
\end{array}} \mu_{j}\left(X_{j_{i}} X_{\beta}+c X_{\xi} X_{j_{i}}\right) \rho_{j}
$$

Again, $\overline{\gamma_{i}} \equiv \overline{X_{j_{i}} \delta_{i}+X_{j_{l}} X_{j_{i}} \theta}$ for some $\delta_{i}, \theta$ and $\overline{\gamma_{i+1}} \equiv \overline{X_{j_{l}} \delta}$ for some $\delta$.

So, we have two representations for $\gamma_{i+1}: \overline{X_{j_{l}} \delta} \equiv \overline{\gamma_{i+1}} \equiv \overline{X_{j_{i}} \delta_{i+1}+X_{j_{l}} X_{j_{i}} \mu}$ So, $X_{j_{i}} \delta_{i+1}+$ $X_{j_{l}}\left(X_{j_{i}} \mu-\delta\right)=\sum_{a=1}^{n} \sum_{j=1}^{n_{a}} \mu_{j}^{a} m_{a} \rho_{j}^{a}+\sum_{j=1}^{n_{\text {sum }}} \mu_{j}\left(X_{j_{i}} X_{\beta}+c X_{\xi} X_{j_{i}}\right) \rho_{j}$ for some monomials $\mu_{j}, \mu_{j}^{a}, \rho_{j}, \rho_{j}^{a}$.

As before, we get that $\overline{\delta_{i+1}} \equiv \overline{X_{j_{i}} \omega+X_{j_{l}} X_{j_{i}} \omega^{\prime}}$ for some $\omega, \omega^{\prime}$. Thus, $\overline{X_{j_{i}} \delta_{i+1}} \equiv \overline{0}$.

Hence, $\overline{\gamma_{i+1}} \equiv \overline{X_{j_{l}} X_{j_{l}} \mu}$.

$\underline{\text { Case } 3}$ Suppose $X_{\xi}=X_{j_{k}}$ for some $k, X_{\beta}=X_{j_{l}}$ for some $l$. Let $\left(\begin{array}{c}\overline{\gamma_{1}} \\ \vdots \\ \overline{\gamma_{n+1}}\end{array}\right) \in \operatorname{ker} \varphi_{2}$. Then $\varphi_{2}\left(\begin{array}{c}\overline{\gamma_{1}} \\ \vdots \\ \overline{\gamma_{n+1}}\end{array}\right)=\left(\begin{array}{c}\sum a_{1 s} \overline{\gamma_{s}} \\ \vdots \\ \sum a_{t s} \overline{\gamma_{s}}\end{array}\right) \equiv\left(\begin{array}{c}\overline{0} \\ \vdots \\ \overline{0}\end{array}\right)$. That is, for all $1 \leq r \leq t, \sum a_{r s} \gamma_{s} \in I$.

For $r=j_{v}, 1 \leq v \leq k-1$ and $k+1 \leq v \leq i-1, \sum a_{j_{v} s} \gamma_{s}=X_{j_{v}} \gamma_{v} \in I$ and $\overline{\gamma_{v}} \equiv \overline{X_{j_{v}} \delta_{v}}$ for some $\delta_{v}$ by Proposition 5.1.

For $r=j_{v}, i+1 \leq v \leq n, \sum a_{j_{v} s} \gamma_{s}=X_{j_{v}} \gamma_{v+1} \in I$ and $\overline{\gamma_{v+1}} \equiv \overline{X_{j_{v}} \delta_{v+1}}$ for some $\delta_{v+1}$ by Proposition 5.1.

For $r=j_{k}, \sum a_{j_{k} s} \gamma_{s}=X_{j_{k}} \gamma_{k}+c X_{j_{i}} \gamma_{i+1} \in I$. Thus, $X_{j_{k}} \gamma_{k}+c X_{j_{i}} \gamma_{i+1}=\sum_{a=1}^{n} \sum_{j=1}^{n_{a}} \mu_{j}^{a} m_{a} \rho_{j}^{a}+$ $\sum_{j=1}^{n_{\text {sum }}} \mu_{j}\left(X_{j_{i}} X_{\beta}+c X_{\xi} X_{j_{i}}\right) \rho_{j}$ for some monomials $\mu_{j}, \mu_{j}^{a}, \rho_{j}, \rho_{j}^{a}$.

Hence,

$$
X_{j_{k}} \gamma_{k}=\sum_{a=1}^{n} \sum_{\substack{\text { subsum } \\
\text { over j }}} \mu_{j}^{a} m_{a} \rho_{j}^{a}+\sum_{\begin{array}{c}
\text { subsum over j } \\
\text { such that } X_{j_{k}} \mid \mu_{j}
\end{array}} \mu_{j}\left(X_{j_{i}} X_{\beta}+c X_{\xi} X_{j_{i}}\right) \rho_{j}+
$$$$
\sum \quad \mu_{j}\left(c X_{j_{k}} X_{j_{i}}\right) \rho_{j}
$$

subsum over $\mathrm{j}$

such that $\mu_{j} \in K \backslash 0$ 
and

$$
\begin{gathered}
c X_{j_{i}} \gamma_{i+1}=\sum_{a=1}^{n} \sum_{\substack{\text { subsum } \\
\text { over j }}} \mu_{j}^{a} m_{a} \rho_{j}^{a}+\sum_{\begin{array}{c}
\text { subsum over j } \\
\text { such that } X_{j_{i}} \mid \mu_{j}
\end{array}} \mu_{j}\left(X_{j_{i}} X_{\beta}+c X_{\xi} X_{j_{i}}\right) \rho_{j}+ \\
\sum_{\substack{\text { subsum over j } \\
\text { such that } \mu_{j} \in K \backslash 0}} \mu_{j}\left(X_{j_{i}} X_{\beta}\right) \rho_{j}
\end{gathered}
$$

So, $\overline{\gamma_{k}} \equiv \overline{X_{j_{k}} \delta_{k}+c X_{j_{i}} \mu}$ and $\overline{\gamma_{i+1}} \equiv \overline{c^{-1} X_{j_{i}} \delta_{i+1}+c^{-1} X_{j_{l}} \mu}$ for some $\delta_{k}, \delta i+1, \mu$.

Lastly, for $r=j_{i}, \sum a_{j_{i} s} \gamma_{s}=X_{j_{i}} \gamma_{i}+X_{j_{l}} \gamma_{i+1} \in I$.

Thus, $X_{j_{i}} \gamma_{i}+X_{j_{l}} \gamma_{i+1}=\sum_{a=1}^{n} \sum_{j=1}^{n_{a}} \mu_{j}^{a} m_{a} \rho_{j}^{a}+\sum_{j=1}^{n_{s u m}} \mu_{j}\left(X_{j_{i}} X_{\beta}+c X_{\xi} X_{j_{i}}\right) \rho_{j}$ for some monomials $\mu_{j}, \mu_{j}^{a}, \rho_{j}, \rho_{j}^{a}$.

$$
\begin{gathered}
\text { Hence, } X_{j_{i}} \gamma_{i}=\sum_{a=1}^{n} \sum_{\begin{array}{c}
\text { subsum } \\
\text { over j }
\end{array}} \mu_{j}^{a} m_{a} \rho_{j}^{a}+\sum_{\begin{array}{c}
\text { subsum over j } \\
\text { such that } X_{j_{i}} \mid \mu_{j}
\end{array}} \mu_{j}\left(X_{j_{i}} X_{\beta}+c X_{\xi} X_{j_{i}}\right) \rho_{j}+ \\
\sum_{\mathcal{I}} \mu_{j}\left(X_{j_{i}} X_{\beta}\right) \rho_{j}
\end{gathered}
$$

where $\mathcal{I}=\left\{j \mid \mu_{j} \in K \backslash 0\right.$ and $\mu_{j} c X_{j_{k}} X_{j_{i}} \rho_{j}$ cancels with a $\mu_{j^{\prime}}^{a^{\prime}} m_{a^{\prime}} \rho_{j^{\prime}}^{a^{\prime}}$ term $\}$

And,

$$
X_{j_{l}} \gamma_{i+1}=\sum_{a=1}^{n} \sum_{\substack{\text { subsum } \\
\text { over j }}} \mu_{j}^{a} m_{a} \rho_{j}^{a}+\sum_{\begin{array}{c}
\text { subsum over j } \\
\text { such that } X_{\beta} \mid \mu_{j}
\end{array}} \mu_{j}\left(X_{j_{i}} X_{\beta}+c X_{\xi} X_{j_{i}}\right) \rho_{j}
$$

Thus, $\overline{\gamma_{i}} \equiv \overline{X_{j_{i}} \delta_{i}+X_{j_{l}} X_{j_{i}} \theta}$ for some $\delta_{i}, \theta$ and $\overline{\gamma_{i+1}} \equiv \overline{X_{j_{l}} \rho}$ for some $\rho$.

So, we have two representations for $\gamma_{i+1}: \overline{X_{j_{l}} \rho} \equiv \overline{\gamma_{i+1}} \equiv \overline{c^{-1} X_{j_{i}} \delta_{i+1}+c^{-1} X_{j_{l}} \mu}$

Thus, $c^{-1} X_{j_{i}} \delta_{i+1}+X_{j_{l}}\left(c^{-1} \underline{\mu-\rho)} \in I\right.$. As above, we get that $\overline{\delta_{i+1}} \equiv \overline{X_{j_{i}} \omega+X_{j_{l}} X_{j_{i}} \omega^{\prime}}$ for some $\omega, \omega^{\prime}$. Which means, $\overline{X_{j_{i}} \delta_{i+1}} \equiv \overline{0}$.

Hence, $\overline{\gamma_{i+1}} \equiv \overline{c^{-1} X_{j_{l}} \mu}$.

Proposition 5.3 Let $\varphi_{2}$ be as defined in Proposition 5.2. Let $\varphi_{3}: \Lambda^{n+2} \longrightarrow \Lambda^{n+1}$ be defined by $\varphi_{3}=\left(b_{r s}\right)$ where 
Case 1: $X_{\xi}=X_{j_{k}}$ for some $k, X_{\beta} \neq X_{j_{l}}$ for all $l$

$$
b_{r s}= \begin{cases}X_{j_{r}} & \text { if } 1 \leq r \leq i \text { and } r=s \\ X_{\beta} X_{j_{i}} & \text { if } r=i, s=i+1 \\ X_{j_{i}}^{2} & \text { if } r=i \text { and } s=i+2 \\ X_{j_{r-1}} & \text { if } i+2 \leq r \leq n+1 \text { and } s=r+1 \\ 0 & \text { if otherwise }\end{cases}
$$

(See Diagram 5.1, Case I, Case 1)

Case 2: $X_{\beta}=X_{j_{l}}$ for some $l$ and $X_{\xi} \neq X_{j_{k}}$ for all $k$.

$$
b_{r s}= \begin{cases}X_{j_{r}} & \text { if } 1 \leq r \leq k \text { and } r=s \\ X_{\beta} X_{j_{i}} & \text { if } r=i+1, s=k+1 \\ X_{j_{r}} & \text { if } k+1 \leq r \leq i \text { and } s=r+1 \\ X_{\beta} X_{j_{i}} & \text { if } r=i \text { and } s=i+2 \\ X_{j_{r-1}} & \text { if } i+2 \leq r \leq n+1 \text { and } s=r+1 \\ 0 & \text { if otherwise }\end{cases}
$$

(See Diagram 5.1, Case I, Case 2)

Case 3: $X_{\xi}=X_{j_{k}}, X_{\beta}=X_{j_{l}}$ for some $k, l$

$$
b_{r s}= \begin{cases}X_{j_{r}} & \text { if } 1 \leq r \leq i \text { and } r=s \\ c^{2} X_{j_{i}} & \text { if } r=k, s=i+1 \\ X_{\beta} & \text { if } r=i+1 \text { and } s=i+1 \\ X_{\beta} X_{j_{i}} & \text { if } r=i \text { and } s=i+2 \\ X_{j_{r-1}} & \text { if } i+2 \leq r \leq n+1 \text { and } s=r+1 \\ 0 & \text { if otherwise }\end{cases}
$$

(See Diagram 5.1, Case I, Case 3)

Then, $\operatorname{ker} \varphi_{2}=i m \varphi_{3}$.

Proof First note that $\varphi_{2} \circ \varphi_{3}=\left(\sum_{w=1}^{n+1} a_{r w} b_{w s}\right)$

$\underline{\text { Case } 1} X_{\xi}=X_{j_{k}}$ for some $k, X_{\beta} \neq X_{j_{l}}$ for all $l$.

For $r=j_{v}, 1 \leq v \leq i$ and $s=v, \sum a_{j_{v} w} b_{w v}=\overline{X_{j_{v}}^{2}} \equiv \overline{0}$.

For $r=j_{i}, s=i+1, \sum a_{j_{i} w} b_{w(i+1)}=\overline{X_{j_{i}} X_{\beta} X_{j_{i}}} \equiv \overline{0}$.

For $r=j_{i}, s=i+2, \sum a_{j_{i} w} b_{w(i+2)}=\overline{X_{j_{i}}^{3}} \equiv \overline{0}$. 
For $r=j_{v}, i+1 \leq v \leq n$ and $s=v+2, \sum a_{j_{v} w} b_{w(v+2)}=\overline{X_{j_{v}}^{2}} \equiv \overline{0}$.

For all other $r, s, \sum a_{r v} b_{v s}=0$. Thus, $\operatorname{im} \varphi_{3} \subseteq \operatorname{ker} \varphi_{2}$.

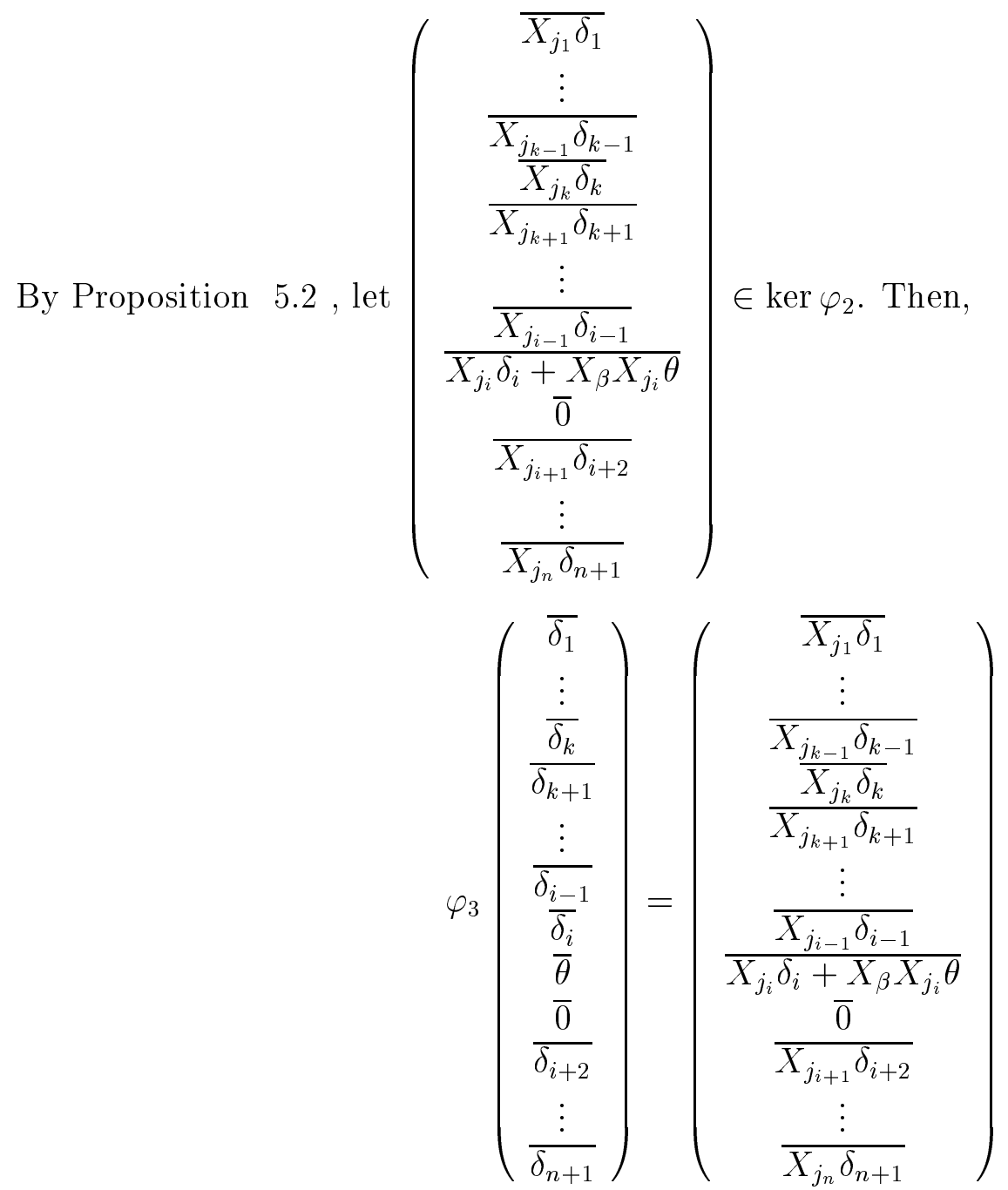

Hence, $\operatorname{ker} \varphi_{2} \subseteq \operatorname{im} \varphi_{3}$.

Case $2 X_{\beta}=X_{j_{l}}$ for some $l$, and $X_{j_{k}}<X_{\xi}<X_{j_{k+1}}$.

For $r=j_{v}, 1 \leq v \leq k$ and $s=v, \sum a_{j_{v} w} b_{w v}=\overline{X_{j_{v}}^{2}} \equiv \overline{0}$.

For $r=j_{v}, k+1 \leq v \leq i$ and $s=v+1, \sum a_{j_{v} w} b_{w(v+1)}=\overline{X_{j_{v}}^{2}} \equiv \overline{0}$.

For $r=j_{i}, s=k+1, \sum a_{j_{i} w} b_{w(k+1)}=\overline{X_{\beta}^{2} X_{j_{i}}} \equiv \overline{0}$.

For $r=j_{i}, s=i+1, \sum a_{j_{i} w} b_{w(i+1)}=\overline{X_{j_{i}}^{2}} \equiv \overline{0}$.

For $r=\xi, s=k+1, \sum a_{\xi w} b_{w(k+1)}=\overline{c X_{j_{i}} X_{\beta} X_{j_{i}}} \equiv \overline{0}$.

For $r=j_{i}, s=i+2, \sum a_{j_{i} w} b_{w(i+2)}=\overline{X_{j_{i}} X_{\beta} X_{j_{i}}} \equiv \overline{0}$. 
For $r=j_{v}, i+1 \leq v \leq n$ and $s=v+2, \sum a_{j_{v} w} b_{w(v+2)}=\overline{X_{j_{v}}^{2}} \equiv \overline{0}$.

For all other $r, s, \sum a_{r v} b_{v s}=0$. Thus, $\operatorname{im} \varphi_{3} \subseteq \operatorname{ker} \varphi_{2}$.

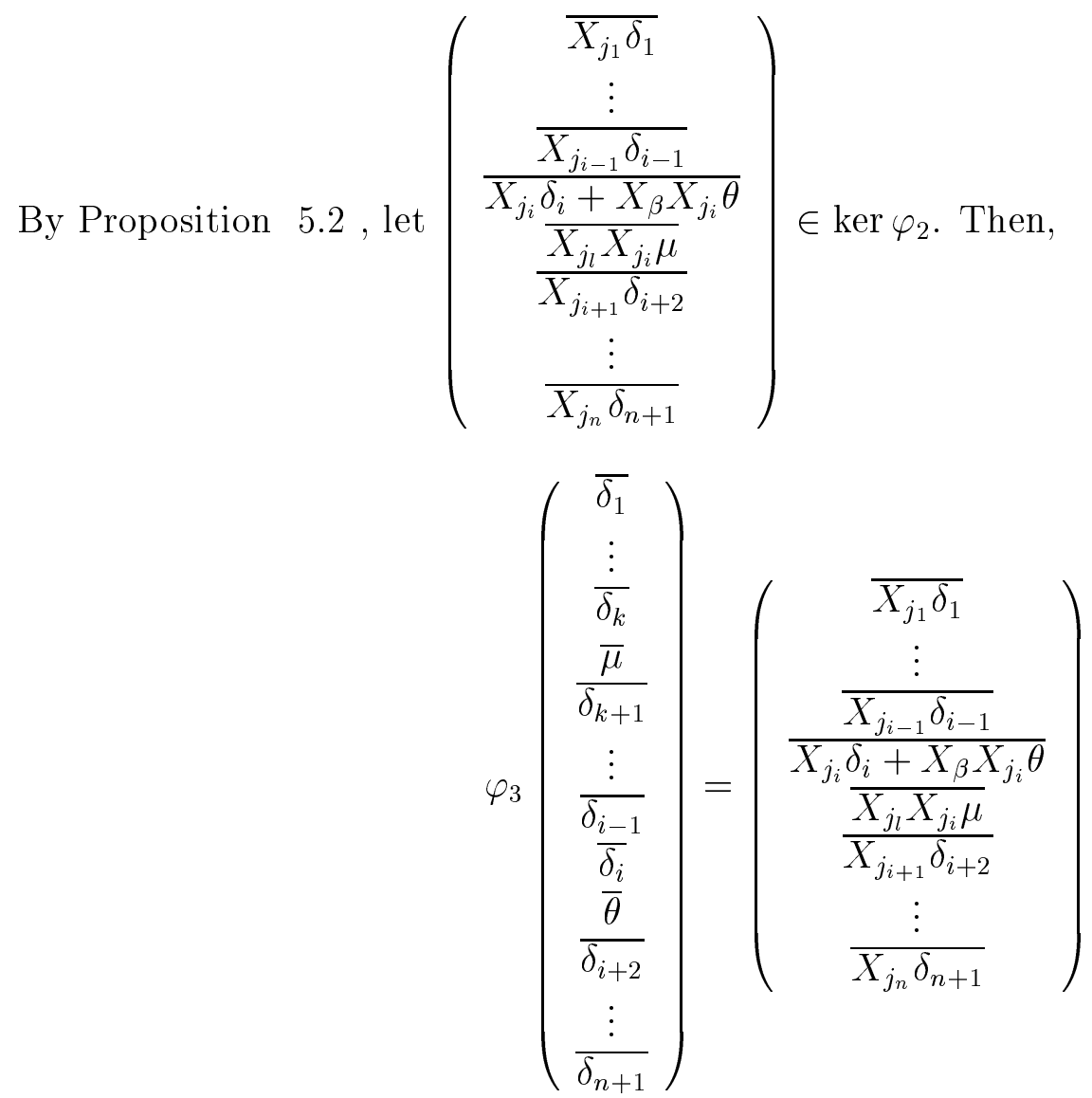

Hence, $\operatorname{ker} \varphi_{2} \subseteq \operatorname{im} \varphi_{3}$.

$\underline{\text { Case } 3} X_{\beta}=X_{j_{l}}$ and $X_{\xi}=X_{j_{k}}$ for some $k, l$.

For $r=j_{v}, 1 \leq v \leq i$ and $s=v, \sum a_{j_{v} w} b_{w v}=\overline{X_{j_{v}}^{2}} \equiv \overline{0}$.

For $r=j_{i}, s=i+1, \sum a_{j_{i} w} b_{w(i+1)}=\overline{X_{\beta}^{2}} \equiv \overline{0}$.

For $r=j_{k}, s=i+1, \sum a_{j_{k} w} b_{w(i+1)}=\overline{c X_{j_{i}} X_{\beta}+c^{2} X_{\xi} X_{j_{i}}} \equiv \overline{0}$.

For $r=j_{i}, s=i+2, \sum a_{j_{i} w} b_{w(i+2)}=\overline{X_{j_{i}} X_{\beta} X_{j_{i}}} \equiv \overline{0}$.

For $r=j_{v}, i+1 \leq v \leq n$ and $s=v+2, \sum a_{j_{v} w} b_{w(v+2)}=\overline{X_{j_{v}}^{2}} \equiv \overline{0}$.

For all other $r, s, \sum a_{r v} b_{v s}=0$. Thus, $\operatorname{im} \varphi_{3} \subseteq \operatorname{ker} \varphi_{2}$. 
By Proposition 5.2 , let $\left(\begin{array}{c}\overline{X_{j_{1}} \delta_{1}} \\ \frac{\vdots}{X_{j_{k-1}} \delta_{k-1}} \\ \frac{X_{j_{k}} \delta_{k}+c X_{j} \mu}{X_{j_{k+1}} \delta_{k+1}} \\ \frac{\vdots}{X_{j_{i-1} \delta_{i-1}}} \\ \frac{X_{j_{i}+X_{\beta} X_{j_{i}} \theta}}{{\frac{c}{X_{j}} X_{j_{l}} \mu}_{\delta_{i+1}}} \\ \frac{\vdots}{X_{j_{n}} \delta_{n}}\end{array}\right) \in \operatorname{ker} \varphi_{2}$. Then,

$$
\varphi_{3}\left(\begin{array}{c}
\overline{\delta_{1}} \\
\vdots \\
\overline{\delta_{k}} \\
\overline{\delta_{k+1}} \\
\frac{\overline{\delta_{i-1}}}{\overline{\delta_{i}}} \\
\overline{c^{-1} \mu} \\
\frac{\bar{\theta}}{\overline{\delta_{i+2}}} \\
\frac{\overline{X_{j_{1}} \delta_{1}}}{\delta_{n+1}}
\end{array}\right)=\left(\begin{array}{c}
\frac{\overline{X_{j_{k-1}} \delta_{k-1}}}{X_{j_{k} \delta_{k}+c X_{j_{i}} \mu}} \\
\frac{X_{j_{k+1}} \delta_{k+1}}{\vdots} \\
\frac{X_{j_{i-1} \delta_{i-1}}}{X_{j_{i}} \frac{\delta_{i}+X_{\beta} X_{j_{i}} \theta}{c^{-1} X_{j_{l}} \mu}} \\
\frac{X_{j_{i+1}} \delta_{i+1}}{\vdots} \\
\frac{\vdots}{X_{j_{n}} \delta_{n}}
\end{array}\right)
$$

Hence, $\operatorname{ker} \varphi_{2} \subseteq \operatorname{im} \varphi_{3}$.

Thus, in all cases, $\operatorname{im} \varphi_{3}=\operatorname{ker} \varphi_{2}$.

Now, we resolve the case when $\operatorname{tip}(p+c q)=X_{\xi} X_{j_{i}}$. For Proposition 5.4, 5.5, suppose tip $(p+c q)=X_{\xi} X_{j_{i}}$. The following result gives a description of the kernel of the last linear map in the resolution of the field for this case. This is followed by Proposition 5.5, showing exactness where the first nonlinear map arises. 
Proposition 5.4 Suppose $m_{1}<\ldots<m_{\gamma}<t i p(p+c q)=X_{\xi} X_{j_{i}}<m_{\gamma+1}<\ldots<m_{n}$. Let $\theta_{2}: \Lambda^{n+1} \longrightarrow \Lambda^{t}$ be defined by $\theta_{2}=\left(a_{r s}\right)$ where

$$
a_{r s}= \begin{cases}X_{j_{s}} & \text { if } 1 \leq s \leq \gamma, r=j_{s} \\ X_{\beta} & \text { if } s=\gamma+1, r=j_{i} \\ c X_{j_{i}} & \text { if } s=\gamma+1, r=\xi \\ X_{j_{s-1}} & \text { if } \gamma+2 \leq s \leq n+1, r=j_{s-1} \\ 0 & \text { if otherwise }\end{cases}
$$

(See Diagram 5.1, Case II)

Then,

$$
\operatorname{ker} \theta_{2}=\left\{\left(\begin{array}{c}
\overline{X_{j_{1}} \delta_{1}} \\
\frac{\overline{X_{j_{i-1}} \delta_{i-1}}}{X_{j_{i} \delta_{i}+X_{\beta} X_{j_{i}} \theta}} \\
\frac{X_{j_{i+1}} \delta_{i+1}}{\vdots} \\
\frac{X_{j_{k-1} \delta_{k-1}}}{\frac{\nabla k}{\nabla k+1}} \\
\frac{X_{j_{k+1} \delta_{k+1}}}{\vdots} \\
\frac{X_{j_{n}} \delta_{n}}{\nabla_{k}}
\end{array}\right) \mid \delta_{1}, \ldots, \delta_{n}, \theta \in K\left\langle X_{1}, \ldots, X_{t}\right\rangle\right\}
$$

where

$$
\nabla_{k}= \begin{cases}0 & \text { if } X_{\xi}=X_{j_{k}} \text { for some } k, X_{\beta} \neq X_{j_{l}} \text { for all } l . \\ X_{j_{k}} \delta_{k} & \text { if } X_{\beta}=X_{j_{l}} \text { for some } l, X_{\xi} \neq X_{j_{k}} \text { for all } k . \\ X_{\beta} \theta^{\prime} & \text { if } X_{\xi}=X_{j_{k}} \text { for some } k, X_{\beta}=X_{j_{l}} \text { for some } l\end{cases}
$$

and

$$
\nabla_{k+1}= \begin{cases}X_{j_{k}} \delta_{k} & \text { if } X_{\xi}=X_{j_{k}} \text { for some } k, X_{\beta} \neq X_{j_{l}} \text { for all } l . \\ X_{\beta} X_{j_{i}} \mu & \text { if } X_{\beta}=X_{j_{l}} \text { for some } l, X_{\xi} \neq X_{j_{k}} \text { for all } k \\ X_{j_{k}} \delta_{k}+c^{-1} X_{j_{i}} \theta^{\prime} & \text { if } X_{\xi}=X_{j_{k}} \text { for some } k, X_{\beta}=X_{j_{l}} \text { for some } l .\end{cases}
$$

$\underline{\text { Proof }} \underline{\text { Case } 1}$ Suppose $X_{\xi}=X_{j_{k}}$ for some $k, X_{\beta} \neq X_{j_{l}}$ for all $l$. Let $\left(\begin{array}{c}\overline{\gamma_{1}} \\ \vdots \\ \overline{\gamma_{n+1}}\end{array}\right) \in \operatorname{ker} \theta_{2}$. Then $\theta_{2}\left(\begin{array}{c}\overline{\gamma_{1}} \\ \vdots \\ \overline{\gamma_{n+1}}\end{array}\right)=\left(\begin{array}{c}\sum a_{1 s} \overline{\gamma_{s}} \\ \vdots \\ \sum a_{t s} \overline{\gamma_{s}}\end{array}\right) \equiv\left(\begin{array}{c}\overline{0} \\ \vdots \\ \overline{0}\end{array}\right)$. That is, for all $1 \leq r \leq t, \sum a_{r s} \gamma_{s} \in I$. 
For $r=j_{v}, 1 \leq v \leq k-1, \sum a_{j_{v}} \gamma_{s}=X_{j_{v}} \gamma_{v} \in I$ and $\overline{\gamma_{v}} \equiv \overline{X_{j_{v}} \delta_{v}}$ for some $\delta_{v}$ by Proposition 5.1 .

For $r=j_{v}, k+1 \leq v \leq n, \sum a_{j_{v} s} \gamma_{s}=X_{j_{v}} \gamma_{v+1} \in I$ and $\overline{\gamma_{v+1}} \equiv \overline{X_{j_{v}} \delta_{v+1}}$ for some $\delta_{v+1}$ by Proposition 5.1.

For $r=j_{k}, \sum a_{j_{k}} \gamma_{s}=X_{j_{k}} \gamma_{k+1}+X_{j_{i}} \gamma_{k} \in I$. Thus, $X_{j_{k}} \gamma_{k+1}+X_{j_{i}} \gamma_{k}=\sum_{a=1}^{n} \sum_{j=1}^{n_{a}} \mu_{j}^{a} m_{a} \rho_{j}^{a}+$ $\sum_{j=1}^{n_{s u m}} \mu_{j}\left(c X_{j_{i}} X_{\beta}+X_{\xi} X_{j_{i}}\right) \rho_{j}$ for some monomials $\mu_{j}, \mu_{j}^{a}, \rho_{j}$, and $\rho_{j}^{a}$.

Hence,

$$
\begin{gathered}
X_{j_{k}} \gamma_{k+1}=\sum_{a=1}^{n} \sum_{\substack{\text { subsum } \\
\text { over j }}} \mu_{j}^{a} m_{a} \rho_{j}^{a}+ \\
\sum_{\substack{\text { subsum over j } \\
\text { such that } X_{j_{k}} \mid \mu_{j}}} \mu_{j}\left(c X_{j_{i}} X_{\beta}+X_{\xi} X_{j_{i}}\right) \rho_{j}+\sum_{\substack{\text { subsum over j } \\
\text { such that } \mu_{j} \in K \backslash 0}} \mu_{j}\left(X_{j_{k}} X_{j_{i}}\right) \rho_{j}
\end{gathered}
$$

and

$$
\begin{gathered}
X_{j_{i}} \gamma_{k}=\sum_{a=1}^{n} \sum_{\substack{\text { subsum } \\
\text { over j }}} \mu_{j}^{a} m_{a} \rho_{j}^{a}+ \\
\sum_{\substack{\text { subsum over j } \\
\text { such that } X_{j_{i}} \mid \mu_{j}}} \mu_{j}\left(c X_{j_{i}} X_{\beta}+X_{\xi} X_{j_{i}}\right) \rho_{j}+\sum_{\substack{\text { subsum over j } \\
\text { such that } \mu_{j} \in K \backslash 0}} c \mu_{j}\left(X_{j_{i}} X_{\beta}\right) \rho_{j}
\end{gathered}
$$

So, $\overline{\gamma_{k+1}} \equiv \overline{X_{j_{k}} \delta_{k}+X_{j_{i}} \theta}$ and $\overline{\gamma_{k}} \equiv \overline{X_{j_{i}} \delta_{i+1}^{\prime}+X_{\beta} X_{j_{i}} \xi^{\prime}+c X_{\beta} \theta}$ for some $\delta_{k+1}, \delta_{k}, \xi, \theta, \xi^{\prime}$.

Lastly, for $r=j_{i}, \sum a_{j_{i} s} \gamma_{s}=X_{j_{i}} \gamma_{i}+c X_{\beta} \gamma_{k} \in I$.

Thus, $X_{j_{i}} \gamma_{i}+c X_{\beta} \gamma_{k}=\sum_{a=1}^{n} \sum_{j=1}^{n_{a}} \mu_{j}^{a} m_{a} \rho_{j}^{a}+\sum_{j=1}^{n_{s u m}} \mu_{j}\left(c X_{j_{i}} X_{\beta}+X_{\xi} X_{j_{i}}\right) \rho_{j}$ for some monomials $\mu_{j}, \mu_{j}^{a}, \rho_{j}, \rho_{j}^{a}$.

$$
\begin{gathered}
\text { And, } X_{j_{i}} \gamma_{i}=\sum_{a=1}^{n} \sum_{\substack{\text { subsum } \\
\text { over j }}} \mu_{j}^{a} m_{a} \rho_{j}^{a}+ \\
\sum_{\substack{\text { subsum over j } \\
\text { such that } X_{j_{i}} \mid \mu_{j}}} \mu_{j}\left(c X_{j_{i}} X_{\beta}+X_{\xi} X_{j_{i}}\right) \rho_{j}+\sum_{\mathcal{I}} \mu_{j}\left(X_{j_{i}} X_{\beta}\right) \rho_{j}
\end{gathered}
$$

where $\mathcal{I}=\left\{j \mid \mu_{j} \in K \backslash 0\right.$ and $\mu_{j} c X_{j_{k}} X_{j_{i}} \rho_{j}$ cancels with a $\mu_{j^{\prime}}^{a^{\prime}} m_{a^{\prime}} \rho_{j^{\prime}}^{a^{\prime}}$ term $\}$ 
And,

$$
c X_{\beta} \gamma_{k}=\sum_{a=1}^{n} \sum_{\substack{\text { subsum } \\
\text { over j }}} \mu_{j}^{a} m_{a} \rho_{j}^{a}+\sum_{\begin{array}{c}
\text { subsum over j } \\
\text { such that } X_{\beta} \mid \mu_{j}
\end{array}} \mu_{j}\left(c X_{j_{i}} X_{\beta}+X_{\xi} X_{j_{i}}\right) \rho_{j}
$$

Thus, $\overline{\gamma_{i}} \equiv \overline{X_{j_{i}} \delta_{i}+X_{\beta} X_{j_{i}} \theta}$ for some $\delta_{i}, \theta$.

And, since $\beta \neq j_{l}$ for all $l, \overline{\gamma_{k}} \equiv \overline{0}$.

So, $\overline{\gamma_{k}} \equiv \overline{0} \equiv \overline{X_{j_{i}} \delta_{i+1}^{\prime}+X_{\beta} X_{j_{i}} \xi^{\prime}+c X_{\beta} \theta}$. Thus, $\overline{X_{j_{i}} \xi^{\prime}+c \theta} \equiv \overline{0}$ which means that $c X_{j_{i}} \theta \in I$. Thus, $\overline{\gamma_{k+1}} \equiv \overline{X_{j_{k}} \delta_{k}}$.

$\underline{\text { Case } 2}$ Suppose $X_{\beta}=X_{j_{l}}$ for some $l, X_{\xi} \neq X_{j_{k}}$ for all $k$. Let $\left(\begin{array}{c}\overline{\gamma_{1}} \\ \vdots \\ \overline{\gamma_{n+1}}\end{array}\right) \in \operatorname{ker} \theta_{2}$. Then $\theta_{2}\left(\begin{array}{c}\overline{\gamma_{1}} \\ \vdots \\ \overline{\gamma_{n+1}}\end{array}\right)=\left(\begin{array}{c}\sum a_{1 s} \overline{\gamma_{s}} \\ \vdots \\ \sum a_{t s} \overline{\gamma_{s}}\end{array}\right) \equiv\left(\begin{array}{c}\overline{0} \\ \vdots \\ \overline{0}\end{array}\right)$. That is, for all $1 \leq r \leq t, \sum a_{r s} \gamma_{s} \in I$.

For $r=j_{v}, 1 \leq v \leq k, v \neq i, \sum a_{j_{v} s} \gamma_{s}=X_{j_{v}} \gamma_{v} \in I$ and $\overline{\gamma_{v}} \equiv \overline{X_{j_{v}} \delta_{v}}$ for some $\delta_{v}$ by Proposition 5.1.

For $r=j_{v}, k+1 \leq v \leq n, \sum a_{j_{v} s} \gamma_{s}=X_{j_{v}} \gamma_{v+1} \in I$ and $\overline{\gamma_{v+1}} \equiv \overline{X_{j_{v}} \delta_{v+1}}$ for some $\delta_{v+1}$ by Proposition 5.1.

For $r=\xi, \sum a_{\xi s} \gamma_{s}=X_{j_{i}} \gamma_{k+1} \in I$. Thus, $X_{j_{i}} \gamma_{k+1}=\sum_{a=1}^{n} \sum_{j=1}^{n_{a}} \mu_{j}^{a} m_{a} \rho_{j}^{a}+\sum_{j=1}^{n_{s u m}} \mu_{j}\left(c X_{j_{i}} X_{\beta}+\right.$ $\left.X_{\xi} X_{j_{i}}\right) \rho_{j}$ for some monomials $\mu_{j}, \mu_{j}^{a}, \rho_{j}, \rho_{j}^{a}$.

Hence,

$$
\begin{gathered}
X_{j_{i}} \gamma_{k+1}=\sum_{a=1}^{n} \sum_{\substack{\text { subsum } \\
\text { over j }}} \mu_{j}^{a} m_{a} \rho_{j}^{a}+ \\
\sum_{\substack{\text { subsum over j } \\
\text { such that } X_{j_{i}} \mid \mu_{j}}} \mu_{j}\left(c X_{j_{i}} X_{\beta}+X_{\xi} X_{j_{i}}\right) \rho_{j}+\sum_{\mathcal{I}} \mu_{j}\left(c X_{j_{i}} X_{\beta}\right) \rho_{j}
\end{gathered}
$$

where $\mathcal{I}=\left\{j \mid \mu_{j} \in K \backslash 0\right.$ and $\mu_{j} X_{\xi} X_{j_{i}} \rho_{j}$ cancels with a $\mu_{j^{\prime}}^{a^{\prime}} \rho_{j^{\prime}}^{a^{\prime}}$ term $\}$

And, $\overline{\gamma_{k+1}} \equiv \overline{X_{j_{i}} \delta^{\prime}+X_{\beta} X_{j_{i}} \mu}$ for some $\delta^{\prime}, \mu$.

Lastly, for $r=j_{i}, \sum a_{j_{i} s} \gamma_{s}=X_{j_{i}} \gamma_{i}+c X_{j_{l}} \gamma_{k+1} \in I$.

Thus, $X_{j_{i}} \gamma_{i}+c X_{j_{l}} \gamma_{k+1}=\sum_{a=1}^{n} \sum_{j=1}^{n_{a}} \mu_{j}^{a} m_{a} \rho_{j}^{a}+\sum_{j=1}^{n_{s u m}} \mu_{j}\left(c X_{j_{i}} X_{\beta}+X_{\xi} X_{j_{i}}\right) \rho_{j}$ for some monomials $\mu_{j}, \mu_{j}^{a}, \rho_{j}, \rho_{j}^{a}$. 


$$
\begin{aligned}
& \text { So, } X_{j_{i}} \gamma_{i}=\sum_{a=1}^{n} \sum_{\substack{\text { subsum } \\
\text { over j }}} \mu_{j}^{a} m_{a} \rho_{j}^{a}+ \\
& \sum_{\substack{\text { subsum over j } \\
\text { such that } X_{j_{i}} \mid \mu_{j}}} \mu_{j}\left(c X_{j_{i}} X_{\beta}+X_{\xi} X_{j_{i}}\right) \rho_{j}+\sum_{\mathcal{I}} \mu_{j}\left(c X_{j_{i}} X_{\beta}\right) \rho_{j}
\end{aligned}
$$

where $\mathcal{I}=\left\{j \mid \mu_{j} \in K \backslash 0\right.$ and $\mu_{j} c X_{\xi} X_{j_{i}} \rho_{j}$ cancels with a $\mu_{j^{\prime}}^{a^{\prime}} m_{a^{\prime}} \rho_{j^{\prime}}^{a^{\prime}}$ term $\}$

And,

$$
X_{j_{l}} \gamma_{k+1}=\sum_{a=1}^{n} \sum_{\substack{\text { subsum } \\
\text { over } \mathrm{j}}} \mu_{j}^{a} m_{a} \rho_{j}^{a}+\sum_{\begin{array}{c}
\text { subsum over j } \\
\text { such that } X_{j_{l}} \mid \mu_{j}
\end{array}} \mu_{j}\left(c X_{j_{i}} X_{\beta}+X_{\xi} X_{j_{i}}\right) \rho_{j}
$$

Again, $\overline{\gamma_{i}} \equiv \overline{X_{j_{i}} \delta_{i}+X_{j_{l}} X_{j_{i}} \theta}$ for some $\delta_{i}, \theta$ and $\overline{\gamma_{k+1}} \equiv \overline{X_{j_{l}} \delta_{k+1}}$ for some $\delta_{k+1}$.

So, we have two representations for $\gamma_{k+1}$ : $\overline{X_{j_{l}} \delta_{k+1}} \equiv \overline{\gamma_{k+1}} \equiv \overline{X_{j_{i}} \delta^{\prime}+X_{j_{l}} X_{j_{i}} \mu}$. Hence, $X_{j_{i}} \delta_{k+1}+X_{j_{l}}\left(X_{j_{i}} \mu-\delta^{\prime}\right)=\sum_{a=1}^{n} \sum_{j=1}^{n_{a}} \mu_{j}^{a} m_{a} \rho_{j}^{a}+\sum_{j=1}^{n_{\text {sum }}} \mu_{j}\left(c X_{j_{i}} X_{\beta}+X_{\xi} X_{j_{i}}\right) \rho_{j}$ for some monomials $\mu_{j}, \mu_{j}^{a}, \rho_{j}, \rho_{j}^{a}$.

As before, we get that $\overline{\delta_{k+1}} \equiv \overline{X_{j_{l}} \theta^{\prime}+X_{j_{i}} \alpha}$. Thus, $\overline{X_{j_{l}} \delta_{k+1}} \equiv \overline{X_{\beta} X_{j_{i}} \theta}$.

Hence, $\overline{\gamma_{k+1}} \equiv \overline{X_{j_{l}} X_{j_{i}} \theta}$.

$\underline{\text { Case } 3}$ Suppose $X_{\xi}=X_{j_{k}}$ for some $k, X_{\beta}=X_{j_{l}}$ for some $l$. Let $\left(\begin{array}{c}\overline{\gamma_{1}} \\ \vdots \\ \overline{\gamma_{n+1}}\end{array}\right) \in \operatorname{ker} \theta_{2}$. Then $\theta_{2}\left(\begin{array}{c}\overline{\gamma_{1}} \\ \vdots \\ \overline{\gamma_{n+1}}\end{array}\right)=\left(\begin{array}{c}\sum a_{1 s} \overline{\gamma_{s}} \\ \vdots \\ \sum a_{t s} \overline{\gamma_{s}}\end{array}\right) \equiv\left(\begin{array}{c}\overline{0} \\ \vdots \\ \overline{0}\end{array}\right)$. That is, for all $1 \leq r \leq t, \sum a_{r s} \gamma_{s} \in I$.

For $r=j_{v}, 1 \leq v \leq k-1, v \neq i, \sum a_{j_{v} s} \gamma_{s}=X_{j_{v}} \gamma_{v} \in I$ and $\overline{\gamma_{v}} \equiv \overline{X_{j_{v}} \delta_{v}}$ for some $\delta_{v}$ by Proposition 5.1.

For $r=j_{v}, k+1 \leq v \leq n, \sum a_{j_{v} s} \gamma_{s}=X_{j_{v}} \gamma_{v+1} \in I$ and $\overline{\gamma_{v+1}} \equiv \overline{X_{j_{v}} \delta_{v+1}}$ for some $\delta_{v+1}$ by Proposition 5.1.

For $r=j_{k}, \sum a_{j_{k} s} \gamma_{s}=X_{j_{k}} \gamma_{k+1}+X_{j_{i}} \gamma_{k} \in I$. Thus, $X_{j_{k}} \gamma_{k+1}+X_{j_{i}} \gamma_{k}=\sum_{a=1}^{n} \sum_{j=1}^{n_{a}} \mu_{j}^{a} m_{a} \rho_{j}^{a}+$ $\sum_{j=1}^{n_{\text {sum }}} \mu_{j}\left(c X_{j_{i}} X_{\beta}+X_{\xi} X_{j_{i}}\right) \rho_{j}$ for some monomials $\mu_{j}, \mu_{j}^{a}, \rho_{j}, \rho_{j}^{a}$. 
Hence,

$$
\begin{gathered}
X_{j_{k}} \gamma_{k+1}=\sum_{a=1}^{n} \sum_{\substack{\text { subsum } \\
\text { over j }}} \mu_{j}^{a} m_{a} \rho_{j}^{a}+ \\
\sum_{\substack{\text { subsum over j } \\
\text { such that } X_{j_{k}} \mid \mu_{j}}} \mu_{j}\left(c X_{j_{i}} X_{\beta}+X_{\xi} X_{j_{i}}\right) \rho_{j}+\sum_{\substack{\text { subsum over j } \\
\text { such that } \mu_{j} \in K \backslash 0}} \mu_{j}\left(c X_{j_{k}} X_{j_{i}}\right) \rho_{j}
\end{gathered}
$$

and

$$
\begin{aligned}
& X_{j_{i}} \gamma_{k}=\sum_{a=1}^{n} \sum_{\substack{\text { subsum } \\
\text { over j }}} \mu_{j}^{a} m_{a} \rho_{j}^{a}+ \\
& \sum \quad \mu_{j}\left(c X_{j_{i}} X_{\beta}+X_{\xi} X_{j_{i}}\right) \rho_{j}+ \\
& \text { subsum over } \mathrm{j} \\
& \text { such that } X_{j_{i}} \mid \mu_{j}
\end{aligned}
$$$$
\text { So, } \overline{\gamma_{k+1}} \equiv \overline{X_{j_{k}} \delta_{k+1}+X_{j_{i}} X_{j_{l}} \xi^{\prime}+X_{j_{i}} \theta^{\prime}} \text { and } \overline{\gamma_{k}} \equiv \overline{X_{j_{i}} \delta+c X_{j_{l}} X_{j_{i}} \xi+c X_{\beta} \theta^{\prime}} \text { for some } \delta_{k}, \delta_{i+1}, \mu \text {. }
$$$$
\text { Lastly, for } r=j_{i}, \sum a_{j_{i} s} \gamma_{s}=X_{j_{i}} \gamma_{i}+c X_{j_{l}} \gamma_{k} \in I \text {. }
$$

Thus, $X_{j_{i}} \gamma_{i}+X_{j_{l}} \gamma_{k}=\sum_{a=1}^{n} \sum_{j=1}^{n_{a}} \mu_{j}^{a} m_{a} \rho_{j}^{a}+\sum_{j=1}^{n_{s u m}} \mu_{j}\left(c X_{j_{i}} X_{\beta}+X_{\xi} X_{j_{i}}\right) \rho_{j}$ for some monomials $\mu_{j}, \mu_{j}^{a}, \rho_{j}, \rho_{j}^{a}$.

$$
\begin{aligned}
& \text { Then, } X_{j_{i}} \gamma_{i}=\sum_{a=1}^{n} \sum_{\begin{array}{c}
\text { subsum } \\
\text { over j }
\end{array}} \mu_{j}^{a} m_{a} \rho_{j}^{a}+ \\
& \sum_{\substack{\text { subsum over j } \\
\text { such that } X_{j_{i}} \mid \mu_{j}}} \mu_{j}\left(c X_{j_{i}} X_{\beta}+X_{\xi} X_{j_{i}}\right) \rho_{j}+\sum_{\mathcal{I}} \mu_{j}\left(c X_{j_{i}} X_{\beta}\right) \rho_{j}
\end{aligned}
$$

where $\mathcal{I}=\left\{j \mid \mu_{j} \in K \backslash 0\right.$ and $\mu_{j} X_{j_{k}} X_{j_{i}} \rho_{j}$ cancels with a $\mu_{j^{\prime}}^{a^{\prime}} m_{a^{\prime}} \rho_{j^{\prime}}^{a^{\prime}}$ term $\}$

And,

$$
X_{j_{l}} \gamma_{k}=\sum_{a=1}^{n} \sum_{\substack{\text { subsum } \\
\text { over j }}} \mu_{j}^{a} m_{a} \rho_{j}^{a}+\sum_{\begin{array}{c}
\text { subsum over j } \\
\text { such that } X_{\beta} \mid \mu_{j}
\end{array}} \mu_{j}\left(c X_{j_{i}} X_{\beta}+X_{\xi} X_{j_{i}}\right) \rho_{j}
$$

Thus, $\overline{\gamma_{i}} \equiv \overline{X_{j_{i}} \delta_{i}+X_{j_{l}} X_{j_{i}} \theta}$ for some $\delta_{i}, \theta$ and $\overline{\gamma_{k}} \equiv \overline{X_{j_{l}} \delta^{\prime}}$ for some $\theta^{\prime}$. 
So, we have two representations for $\gamma_{k}$ and we thus obtain $\overline{X_{j_{i}} \delta} \equiv \overline{0}$.

Similarly, by looking at $X_{j_{i}} \gamma_{k}+X_{j_{k}} \gamma_{k+1} \in I$, we get that $\overline{X_{j_{i} \delta^{\prime}}} \equiv \overline{c X_{j_{i} \theta^{\prime}}}$. Lastly, setting $\delta_{k}=\delta_{k+1}+c^{-1} X_{j_{i}} \xi^{\prime}$, we see that $\overline{\gamma_{k+1}} \equiv \overline{X_{j_{k}} \delta_{k}+c^{-1} X_{j_{i} \delta^{\prime}}}$.

Finally, for $\operatorname{tip}(p+c q)=X_{\xi} X_{j_{i}}$, we show the resolution is exact at the point of nonlinearity.

Proposition 5.5 Let $\theta_{2}$ be as defined in Proposition 5.4. Let $\theta_{3}=\left(b_{r s}\right)$ where

Case 1: $X_{\xi}=X_{j_{k}}$ for some $k, X_{\beta} \neq X_{j_{l}}$ for all $l$

$$
b_{r s}= \begin{cases}X_{j_{r}} & \text { if } 1 \leq r \leq i \text { and } r=s \\ X_{\beta} X_{j_{i}} & \text { if } r=i, s=i+1 \\ X_{\beta}^{2} & \text { if } r=i \text { and } s=i+2 \\ X_{j_{r}} & \text { if } i+1 \leq r \leq k-1 \text { and } s=r+2 \\ X_{j_{r-1}} & \text { if } k+1 \leq r \leq n+1 \text { and } s=r+1 \\ 0 & \text { if otherwise }\end{cases}
$$

(See Diagram 5.1, Case II, Case 1)

Case 2: $X_{\beta}=X_{j_{l}}$ for some $l$ and $X_{\xi} \neq X_{j_{k}}$ for all $k$.

$$
b_{r s}= \begin{cases}X_{j_{r}} & \text { if } 1 \leq r \leq i \text { and } r=s \\ X_{\beta} X_{j_{i}} & \text { if } r=i, s=r+1 \\ X_{j_{r}} & \text { if } i+1 \leq r \leq k \text { and } s=r+1 \\ X_{\beta} X_{j_{i}} & \text { if } r=k+1 \text { and } s=r+1 \\ X_{j_{r-1}} & \text { if } k+2 \leq r \leq n+1 \text { and } s=r+1 \\ 0 & \text { if otherwise }\end{cases}
$$

(See Diagram 5.1, Case II, Case 2)

Case 3: $X_{\xi}=X_{j_{k}}, X_{\beta}=X_{j_{l}}$ for some $k, l$

$$
b_{r s}= \begin{cases}X_{j_{r}} & \text { if } 1 \leq r \leq i \text { and } r=s \\ X_{\beta} X_{j_{i}} & \text { if } r=i \text { and } s=i+1 \\ X_{j_{r}} & \text { if } i+1 \leq r \leq k-1 \text { and } s=r+1 \\ c X_{\beta} & \text { if } r=k \text { and } s=k+1 \\ X_{j_{i}} & \text { if } r=k+1=s \\ X_{j_{r-1}} & \text { if } k+1 \leq r \leq n+1 \text { and } s=r+1 \\ 0 & \text { if otherwise }\end{cases}
$$


(See Diagram 5.1, Case II, Case 3)

Then, $\operatorname{ker} \theta_{2}=i m \theta_{3}$.

Proof First note that $\theta_{2} \circ \theta_{3}=\left(\sum_{w=1}^{n+1} a_{r w} b_{w s}\right)$

Case $1 X_{\xi}=X_{j_{k}}$ for some $k, X_{\beta} \neq X_{j_{l}}$ for all $l$.

For $r=j_{v}, 1 \leq v \leq i$ and $s=v, \sum a_{j_{v} w} b_{w v}=\overline{X_{j_{v}}^{2}} \equiv \overline{0}$.

For $r=j_{i}, s=i+1, \sum a_{j_{i} w} b_{w(i+1)}=\overline{X_{j_{i}} X_{\beta} X_{j_{i}}} \equiv \overline{0}$.

For $r=j_{i}, s=i+2, \sum a_{j_{i} w} b_{w(i+2)}=\overline{X_{j_{i}} X_{\beta}^{2}} \equiv \overline{0}$.

For $r=j_{v}, i+1 \leq v \leq k-1$ and $s=v+2, \sum a_{j_{v} w} b_{w(v+2)}=\overline{X_{j_{v}}^{2}} \equiv \overline{0}$.

For $r=j_{v}, k \leq v \leq n$ and $s=v+2, \sum a_{j_{v} w} b_{w(v+2)}=\overline{X_{j_{v}}^{2}} \equiv \overline{0}$.

For all other $r, s, \sum a_{r v} b_{v s}=0$. Thus, $\operatorname{im} \theta_{3} \subseteq \operatorname{ker} \theta_{2}$. 


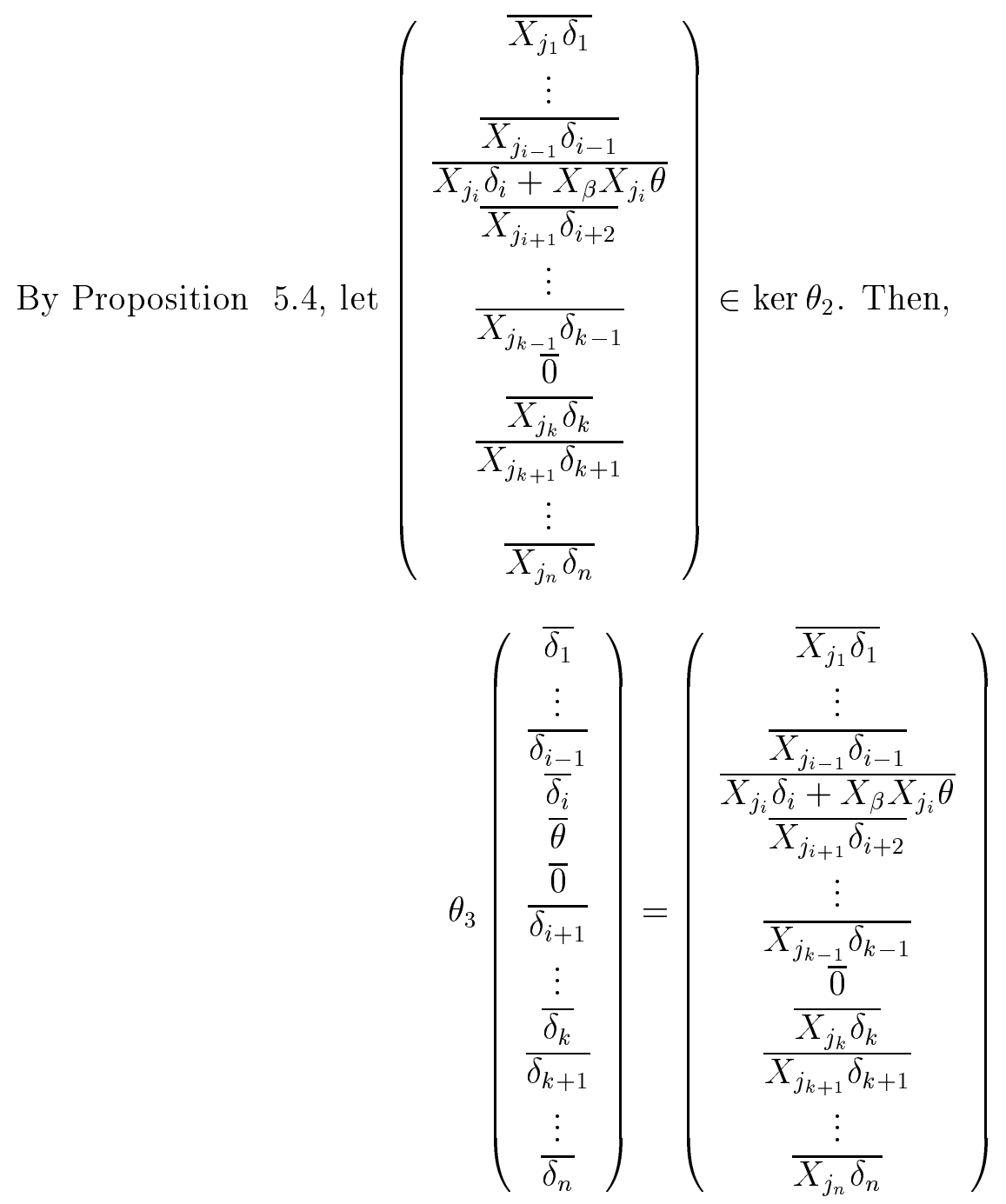

Hence, $\operatorname{ker} \theta_{2} \subseteq \operatorname{im} \theta_{3}$.

Case $2 X_{\beta}=X_{j_{l}}$ for some $l$, and $X_{j_{k}}<X_{\xi}<X_{j_{k+1}}$.

For $r=j_{v}, 1 \leq v \leq i$ and $s=v, \sum a_{j_{v} w} b_{w v}=\overline{X_{j_{v}}^{2}} \equiv \overline{0}$.

For $r=j_{v}, i+1 \leq v \leq k$ and $s=v+1, \sum a_{j_{v} w} b_{w(v+1)}=\overline{X_{j_{v}}^{2}} \equiv \overline{0}$.

For $r=j_{i}, s=k+2, \sum a_{j_{i} w} b_{w(k+2)}=\overline{X_{\beta}^{2} X_{j_{i}}} \equiv \overline{0}$.

For $r=j_{i}, s=i+1, \sum a_{j_{i} w} b_{w(i+1)}=\overline{X_{j_{i}} X_{\beta} X_{j_{i}}} \equiv \overline{0}$.

For $r=\xi, s=k+2, \sum a_{\xi w} b_{w(k+2)}=\overline{X_{j_{i}} X_{\beta} X_{j_{i}}} \equiv \overline{0}$.

For $r=j_{v}, k+1 \leq v \leq n$ and $s=v+2, \sum a_{j_{v} w} b_{w(v+2)}=\overline{X_{j_{v}}^{2}} \equiv \overline{0}$.

For all other $r, s, \sum a_{r v} b_{v s}=0$. Thus, $\operatorname{im} \theta_{3} \subseteq \operatorname{ker} \theta_{2}$. 


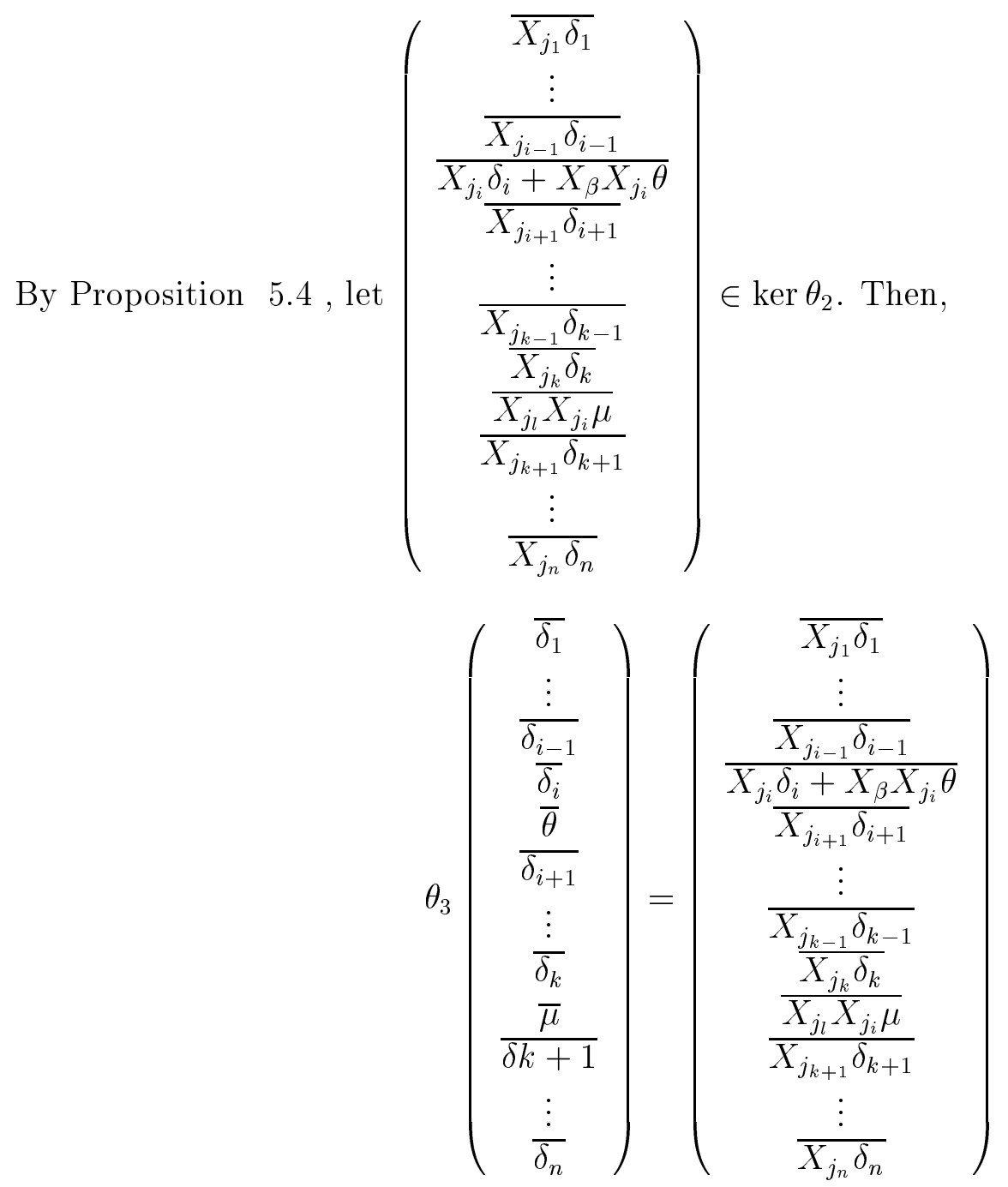

Hence, $\operatorname{ker} \theta_{2} \subseteq \operatorname{im} \theta_{3}$.

$\underline{\text { Case } 3} X_{\beta}=X_{j_{l}}$ and $X_{\xi}=X_{j_{k}}$ for some $k, l$.

For $r=j_{v}, 1 \leq v \leq i$ and $s=v, \sum a_{j_{v} w} b_{w v}=\overline{X_{j_{v}}^{2}} \equiv \overline{0}$.

For $r=j_{i}, s=i+1, \sum a_{j_{i} w} b_{w(i+1)}=\overline{X_{j_{i}} X_{\beta} X_{j_{i}}} \equiv \overline{0}$.

For $r=j_{k}, s=k+1, \sum a_{j_{k} w} b_{w(k+1)}=\overline{c X_{j_{i}} X_{\beta}+X_{\xi} X_{j_{i}}} \equiv \overline{0}$.

For $r=j_{i}, s=k+1, \sum a_{j_{i} w} b_{w(k+1)}=\overline{c^{2} X_{\beta}^{2}} \equiv \overline{0}$.

For $r=j_{v}, i+1 \leq v \leq k-1$ and $s=v+1, \sum a_{j_{v} w} b_{w(v+1)}=\overline{X_{j_{v}}^{2}} \equiv \overline{0}$.

For $r=j_{v}, k \leq v \leq n$ and $s=v+2, \sum a_{j_{v} w} b_{w(v+2)}=\overline{X_{j_{v}}^{2}} \equiv \overline{0}$.

For all other $r, s, \sum a_{r v} b_{v s}=0$. Thus, $\operatorname{im} \theta_{3} \subseteq \operatorname{ker} \theta_{2}$. 


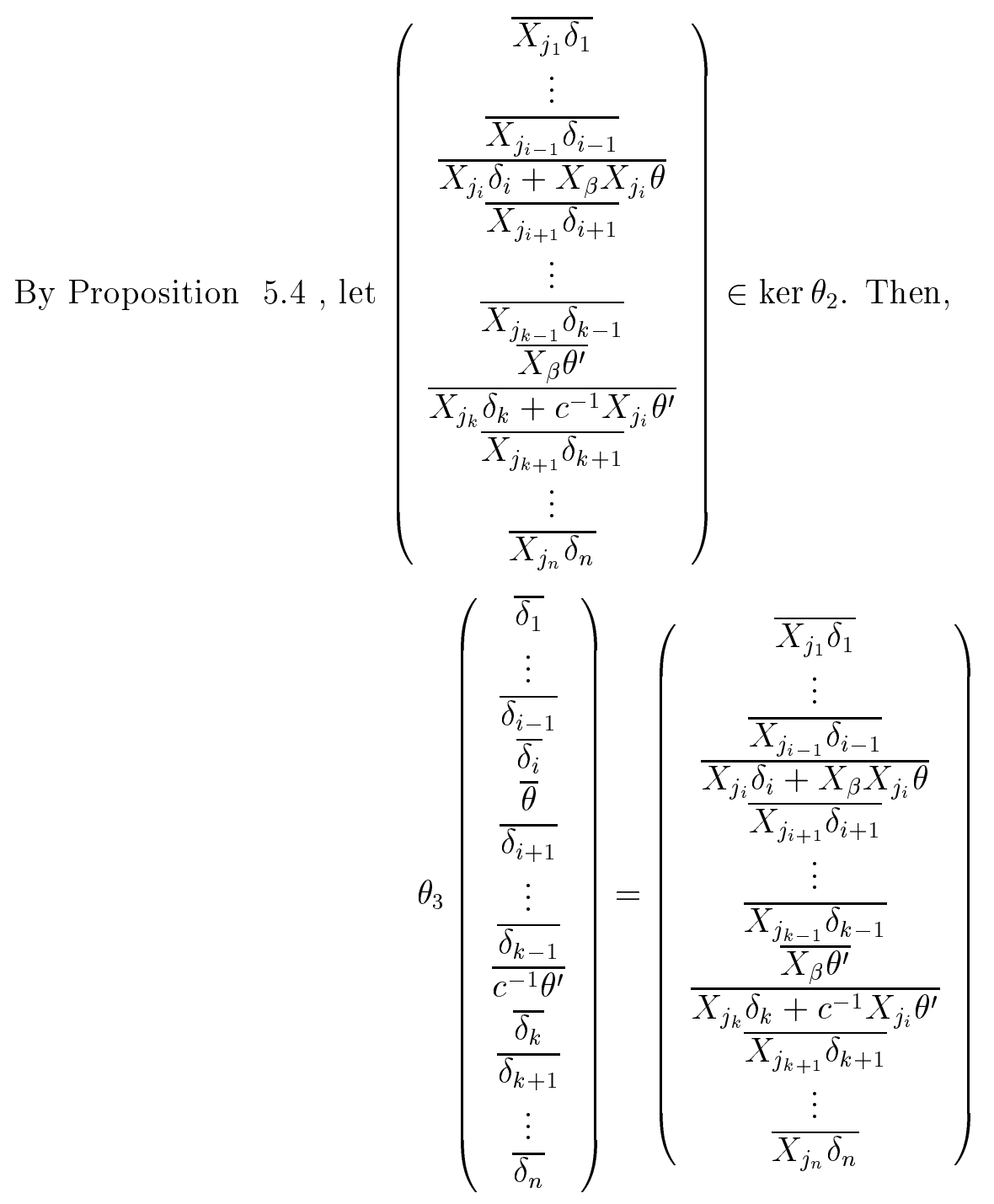

Hence, $\operatorname{ker} \theta_{2} \subseteq \operatorname{im} \theta_{3}$.

Thus, in all cases, $\operatorname{im} \theta_{3}=\operatorname{ker} \theta_{2}$.

Theorem 5.1 Let $I=\left\langle m_{1}, \ldots, m_{n}, p+c q\right\rangle$ be an ideal in $K\left\langle X_{1}, \ldots, X_{t}\right\rangle$ where $p+c q=$ $X_{j_{i}} X_{\beta}+c X_{\xi} X_{j_{i}}$ for some $1 \leq i \leq n$ and $\beta \neq \xi, \beta, \xi \neq j_{i}$. Then, $\Lambda=K\left\langle X_{1}, \cdots, X_{t}\right\rangle / I$ is not Koszul.

$\underline{\text { Proof }}$

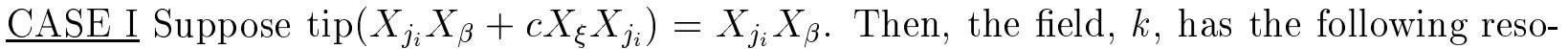
lution:

$$
\cdots \longrightarrow \Lambda^{n+2} \stackrel{\varphi_{3}}{\longrightarrow} \Lambda^{n+1} \stackrel{\varphi_{2}}{\longrightarrow} \Lambda^{t} \stackrel{\left(X_{1} \stackrel{\cdots}{\longrightarrow} X_{t}\right)}{\longrightarrow} \Lambda \longrightarrow k \longrightarrow 0
$$


where $\varphi_{2}, \varphi_{3}$ are as defined in Proposition 5.2 and Proposition 5.3.

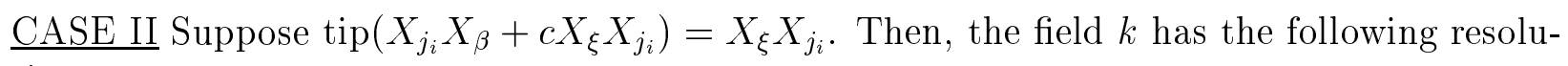
tion:

$$
\cdots \longrightarrow \Lambda^{n+2} \stackrel{\theta_{3}}{\longrightarrow} \Lambda^{n+1} \stackrel{\theta_{2}}{\longrightarrow} \Lambda^{t}\left(X_{1} \stackrel{\cdots}{\longrightarrow} X_{t}\right) \Lambda \longrightarrow k \longrightarrow 0
$$

where $\theta_{2}, \theta_{3}$ are as defined in Proposition 5.4 and Proposition 5.5.

Thus, $\left(\begin{array}{c}\overline{0} \\ \vdots \\ \frac{\overline{0}}{X_{\beta} X_{j_{i}}} \\ \overline{0} \\ \vdots \\ \overline{0}\end{array}\right) \in \operatorname{ker} \varphi_{2}$ and $\operatorname{ker} \theta_{2}$. So,

$$
\Lambda^{n+1} \stackrel{\varphi_{2}}{\longrightarrow} \Lambda^{t} \stackrel{\left(X_{1} \stackrel{\cdots}{\longrightarrow} X_{t}\right)}{\longrightarrow} \Lambda \longrightarrow k \longrightarrow 0
$$

and

$$
\Lambda^{n+1} \stackrel{\theta_{2}}{\longrightarrow} \Lambda^{t}\left(X_{1} \stackrel{\cdots}{\longrightarrow} X_{t}\right) \Lambda \longrightarrow k \longrightarrow 0
$$

are linear resolutions of length 2 . If we were to extend to length 3 by any map $\varphi_{3}^{\prime}$ or $\theta_{3}^{\prime}$ and projective module $P_{3}$, then the image of this new map must equal $\operatorname{ker} \varphi_{2}$ or $\operatorname{ker} \theta_{2}$ and hence would be nonlinear. 


\section{Chapter 6}

\section{Additional Classes of Koszul Algebras}

The duality between a ring and its opposite is well known. This duality allows much to be concluded about a ring's opposite since a ring and its opposite share many properties. In fact, the opposite algebra of a Koszul algebra is also Koszul. [6] By selecting suitably restricted ideals from the results of the previous chapters and looking at the opposite algebras, we easily obtain results for more general quadratic algebras.

Proposition 6.1 $\Lambda=K\left\langle X_{1}, \ldots, X_{t}\right\rangle /\left\langle X_{i} X_{l}+c X_{i} X_{k}, \hat{p_{\alpha \beta}}, \hat{m_{1}}, \ldots \hat{m_{n}}\right\rangle$ for $i, j$, and $k$ distinct, $\hat{m}_{j} \neq X_{i}^{2}, X_{k}^{2}, X_{l}^{2}$ a perfect square monomial for all $j$, and $\hat{p_{\alpha \beta}}=X_{\alpha} X_{\beta} \neq X_{i} X_{l}, X_{i} X_{k}$ with $\alpha \neq \beta$ ranging from $1, \ldots, t$, is Koszul.

Proof We remark that for $I$ described in Theorem 4.1, $\Lambda^{o p}$ has dual basis

$$
\left\{\left(X_{\gamma}^{2}\right)^{*},\left(X_{\alpha} X_{\beta}\right)^{*},\left(X_{j_{i}} X_{j_{l}}\right)^{*}-c\left(X_{j_{i}} X_{j_{k}}\right)^{*} \mid \gamma \neq j_{s} \forall s, \alpha \neq \beta, \text { and } X_{\alpha} X_{\beta} \neq X_{j_{i}} X_{j_{l}}, X_{j_{i}} X_{j_{k}}\right\}
$$

Proposition $6.2 \Lambda=K\left\langle X_{1}, \ldots, X_{t}\right\rangle /\left\langle X_{l} X_{i}+c X_{k} X_{i}, \hat{p_{\alpha \beta}}, \hat{m_{1}}, \ldots \hat{m_{n}}\right\rangle$ for $i, j$, and $k$ distinct, $\hat{m}_{j} \neq X_{i}^{2}, X_{k}^{2}, X_{l}^{2}$ a perfect square monomial for all $j$, and $\hat{p_{\alpha \beta}}=X_{\alpha} X_{\beta} \neq X_{i} X_{l}, X_{i} X_{k}$ with $\alpha \neq \beta$ ranging from $1, \ldots, t$, is Koszul.

Proof We remark that for $I$ described in Theorem $4.2, \Lambda^{o p}$ has dual basis

$$
\left\{\left(X_{\gamma}^{2}\right)^{*},\left(X_{\alpha} X_{\beta}\right)^{*},\left(X_{j_{l}} X_{j_{i}}\right)^{*}-c\left(X_{j_{k}} X_{j_{i}}\right)^{*} \mid \gamma \neq j_{s} \forall s, \alpha \neq \beta, \text { and } X_{\alpha} X_{\beta} \neq X_{j_{l}} X_{j_{i}}, X_{j_{k}} X_{j_{l}}\right\}
$$


Proposition $6.3 \Lambda=K\left\langle X_{1}, \ldots, X_{t}\right\rangle /\left\langle X_{i} X_{l}+c X_{i} X_{k}, \hat{p_{\alpha \beta}}, \hat{m_{1}}, \ldots \hat{m_{n}}\right\rangle$ for $i, j$, and $k$ distinct, $\hat{m}_{j} \neq X_{i}^{2}, X_{k}^{2}$ a perfect square monomial for all $j$, and $\hat{p_{\alpha \beta}}=X_{\alpha} X_{\beta} \neq X_{i} X_{l}, X_{i} X_{k}$ with $\alpha$ and $\beta$ ranging from $1, \ldots, t$, is Koszul.

Proof We remark that for $I$ described in Theorem 3.2, $\Lambda^{o p}$ has dual basis

$$
\left\{\left(X_{\xi}^{2}\right)^{*},\left(X_{\alpha} X_{\theta}\right)^{*},\left(X_{j_{i}} X_{\gamma}\right)^{*}-c\left(X_{j_{i}} X_{\beta}\right)^{*} \mid \xi \neq j_{s} \forall s, \alpha \neq \theta, \text { and } X_{\alpha} X_{\theta} \neq X_{j_{i}} X_{\gamma}, X_{j_{i}} X_{\beta}\right\}
$$

Proposition $6.4 \Lambda=K\left\langle X_{1}, \ldots, X_{t}\right\rangle /\left\langle X_{l} X_{i}+c X_{k} X_{i}, \hat{p_{\alpha \beta}}, \hat{m_{1}}, \ldots \hat{m_{n}}\right\rangle$ for $i, j$, and $k$ distinct, $\hat{m}_{j} \neq X_{i}^{2}, X_{k}^{2}$ a perfect square monomial for all $j$, and $\hat{p_{\alpha \beta}}=X_{\alpha} X_{\beta} \neq X_{i} X_{l}, X_{i} X_{k}$ with $\alpha$ and $\beta$ ranging from $1, \ldots, t$, is Koszul.

Proof We remark that for $I$ described in Theorem $3.1, \Lambda^{o p}$ has dual basis

$$
\left\{\left(X_{\xi}^{2}\right)^{*},\left(X_{\alpha} X_{\theta}\right)^{*},\left(X_{\gamma} X_{j_{i}}\right)^{*}-c\left(X_{\beta} X_{j_{i}}\right)^{*} \mid \xi \neq j_{s} \forall s, \alpha \neq \theta \text {, and } X_{\alpha} X_{\theta} \neq X_{\gamma} X_{j_{i}}, X_{\beta} X_{j_{i}}\right\}
$$

Proposition $6.5 \Lambda=K\left\langle X_{1}, \ldots, X_{t}\right\rangle /\left\langle X_{i} X_{\xi}+c X_{\xi} X_{i}, \hat{p_{\alpha \beta}}, \hat{m}_{1}, \ldots \hat{m}_{n}\right\rangle$ for $i \neq \xi, \hat{m}_{j} \neq X_{i}^{2}$ a perfect square monomial for all $j$, and $\hat{p_{\alpha \beta}}=X_{\alpha} X_{\beta} \neq X_{i} X_{\xi}, X_{\xi} X_{i}$ with $\alpha$ and $\beta$ ranging from $1, \ldots, t$, is Koszul.

Proof We remark that for $I$ described in Lemma $3.2, \Lambda^{o p}$ has dual basis

$$
\left\{\left(X_{\xi}^{2}\right)^{*},\left(X_{\alpha} X_{\theta}\right)^{*},\left(X_{\beta} X_{j_{i}}\right)^{*}-c\left(X_{j_{i}} X_{\beta}\right)^{*} \mid \xi \neq j_{s} \forall s, \alpha \neq \theta, \text { and } X_{\alpha} X_{\theta} \neq X_{j_{i}} X_{\beta}, X_{\beta} X_{j_{i}}\right\}
$$

Here we also note that we have a similar situation as above with nonKoszul algebras. That is, the duals of nonKoszul algebras produce nonKoszul algebras. Thus, we obtain the following class of algebras which are not Koszul:

Proposition $6.6 \Lambda=K\left\langle X_{1}, \ldots, X_{t}\right\rangle /\left\langle X_{i} X_{l}+c X_{k} X_{i}, \hat{p_{\alpha \beta}}, \hat{m_{1}}, \ldots \hat{m}_{n}\right\rangle$ for $i, j$, and $k$ distinct, $\hat{m}_{j} \neq X_{i}^{2}$ a perfect square monomial for all $j$, and $\hat{p_{\alpha \beta}}=X_{\alpha} X_{\beta} \neq X_{i} X_{l}, X_{i} X_{k}$ with $\alpha \neq$ beta ranging from $1, \ldots, t$, is not Koszul.

Proof We remark that for $I$ described in Theorem 5.1, $\Lambda^{o p}$ has dual basis

$$
\left\{\left(X_{\gamma}^{2}\right)^{*},\left(X_{\alpha} X_{\beta}\right)^{*},\left(X_{\xi} X_{j_{i}}\right)^{*}-c\left(X_{j_{i}} X_{\beta}\right)^{*} \mid \gamma \neq j_{s} \forall s, \alpha \neq \beta \text {, and } X_{\alpha} X_{\beta} \neq X_{\xi} X_{j_{i}}, X_{j_{i}} X_{j_{k}}\right\}
$$




\section{Chapter 7}

\section{Matrix Diagrams}

To elucidate the maps of the resolutions developed in Chapters 3, 4, and 5, we now provide diagrams of the associated matrices. The diagram numbers correspond to the theorem numbers from the previous chapters. That is, for example, Diagram 3.1 clarifies the matrices associated with the maps of the linear resolution of the field as defined in Theorem 3.1.

\section{Diagram 3.1}

$$
\varphi=\left(\begin{array}{cccccccc}
X_{j_{1}} & 0 & \cdots & 0 & 0 & 0 & \cdots & 0 \\
0 & X_{j_{2}} & \cdots & 0 & 0 & 0 & \cdots & 0 \\
\vdots & \vdots & \ddots & \vdots & \vdots & \vdots & \ddots & \vdots \\
0 & 0 & \cdots & X_{j_{l}} & 0 & 0 & \cdots & 0 \\
0 & 0 & \cdots & 0 & X_{j_{i}} & 0 & \cdots & 0 \\
0 & 0 & \cdots & 0 & 0 & X_{j_{l+1}} & \cdots & 0 \\
\vdots & \vdots & \ddots & \vdots & \vdots & \vdots & \ddots & \vdots \\
0 & 0 & \cdots & 0 & 0 & 0 & \cdots & X_{j_{n}}
\end{array}\right)
$$


Diagram 3.1, cont.

$$
\begin{aligned}
& \begin{array}{llllllllllll}
1 & 0 & 0 & \cdots & 0 & 0 & \cdots & 0 & 0 & 0 & \cdots & 0 \\
\vdots & \vdots & \vdots & \ddots & \vdots & \vdots & \ddots & \vdots & \vdots & \vdots & \ddots & \vdots \\
j_{1} & X_{j_{1}} & 0 & \cdots & 0 & 0 & \cdots & 0 & 0 & 0 & \cdots & 0
\end{array} \\
& \begin{array}{l|ccccccccccc}
\vdots & \vdots & \vdots & \ddots & \vdots & \vdots & \ddots & \vdots & \vdots & \vdots & \ddots & \vdots
\end{array} \\
& \begin{array}{l|ccccccccccc}
\vdots & \vdots & \vdots & \ddots & \vdots & \vdots & \ddots & \vdots & \vdots & \vdots & \ddots & \vdots \\
j_{k} & 0 & 0 & \cdots & X_{j_{k}} & 0 & \cdots & 0 & 0 & 0 & \cdots & 0
\end{array} \\
& \begin{array}{l|lllllllllll}
\vdots & \vdots & \vdots & \ddots & \vdots & \vdots & \ddots & \vdots & \vdots & \vdots & \ddots & \vdots \\
\beta & 0 & 0 & \cdots & 0 & 0 & & & &
\end{array}
\end{aligned}
$$

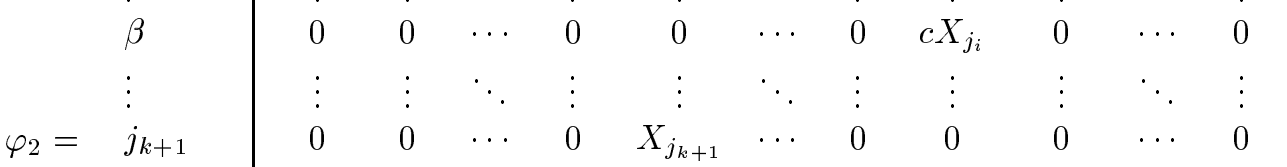

$$
\begin{aligned}
& \begin{array}{l|ccccccccccc}
\vdots & \vdots & \vdots & \ddots & \vdots & \vdots & \ddots & \vdots & \vdots & \vdots & \ddots & \vdots \\
j_{l} & 0 & 0 & \cdots & 0 & 0 & \cdots & X_{j_{l}} & 0 & 0 & & 0
\end{array} \\
& \begin{array}{l|ccccccccccc}
\vdots & \vdots & \vdots & \ddots & \vdots & \vdots & \ddots & \vdots & \vdots & \vdots & \ddots & \vdots \\
\gamma & 0 & 0 & \cdots & 0 & 0 & \cdots & 0 & X_{j_{i}} & 0 & & 0
\end{array}
\end{aligned}
$$

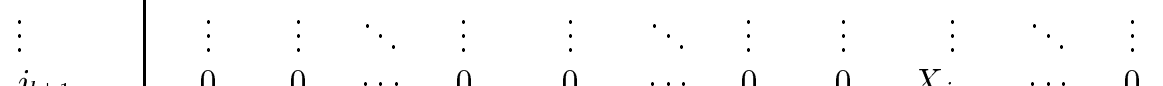

$$
\begin{aligned}
& \begin{array}{llllllllllll}
j_{l+1} & 0 & 0 & \cdots & 0 & 0 & \cdots & 0 & 0 & X_{j_{l+1}} & \cdots & 0
\end{array} \\
& \begin{array}{l|ccccccccccc}
\vdots & \vdots & \vdots & \ddots & \vdots & \vdots & \ddots & \vdots & \vdots & \vdots & \ddots & \vdots \\
j_{n} & 0 & 0 & \cdots & 0 & 0 & \cdots & 0 & 0 & 0 & \cdots & X_{j_{n}}
\end{array}
\end{aligned}
$$

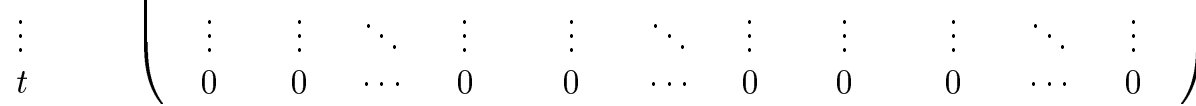




\section{Diagram 3.2}

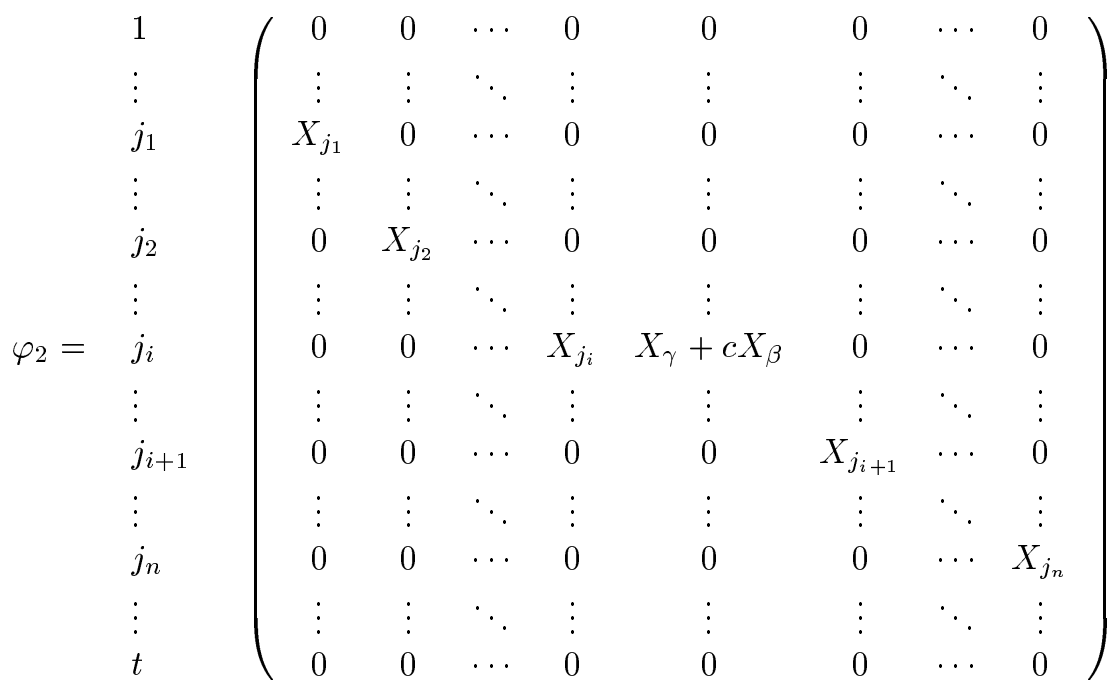

$$
\begin{aligned}
& \varphi=\left(\begin{array}{cccccccc}
X_{j_{1}} & 0 & \cdots & 0 & 0 & 0 & \cdots & 0 \\
0 & X_{j_{2}} & \cdots & 0 & 0 & 0 & \cdots & 0 \\
\vdots & \vdots & \ddots & \vdots & \vdots & \vdots & \ddots & \cdots \\
0 & 0 & \cdots & X_{j_{i}} & X_{\gamma}+c X_{\beta} & 0 & \cdots & 0 \\
0 & 0 & \cdots & 0 & 0 & 0 & \cdots & 0 \\
0 & 0 & \cdots & 0 & 0 & X_{j_{i+1}} & \cdots & 0 \\
\vdots & \vdots & \ddots & \vdots & \vdots & \vdots & \ddots & \cdots \\
0 & 0 & \cdots & 0 & 0 & 0 & \cdots & X_{j_{n}}
\end{array}\right)
\end{aligned}
$$




\section{Diagram 3.3}

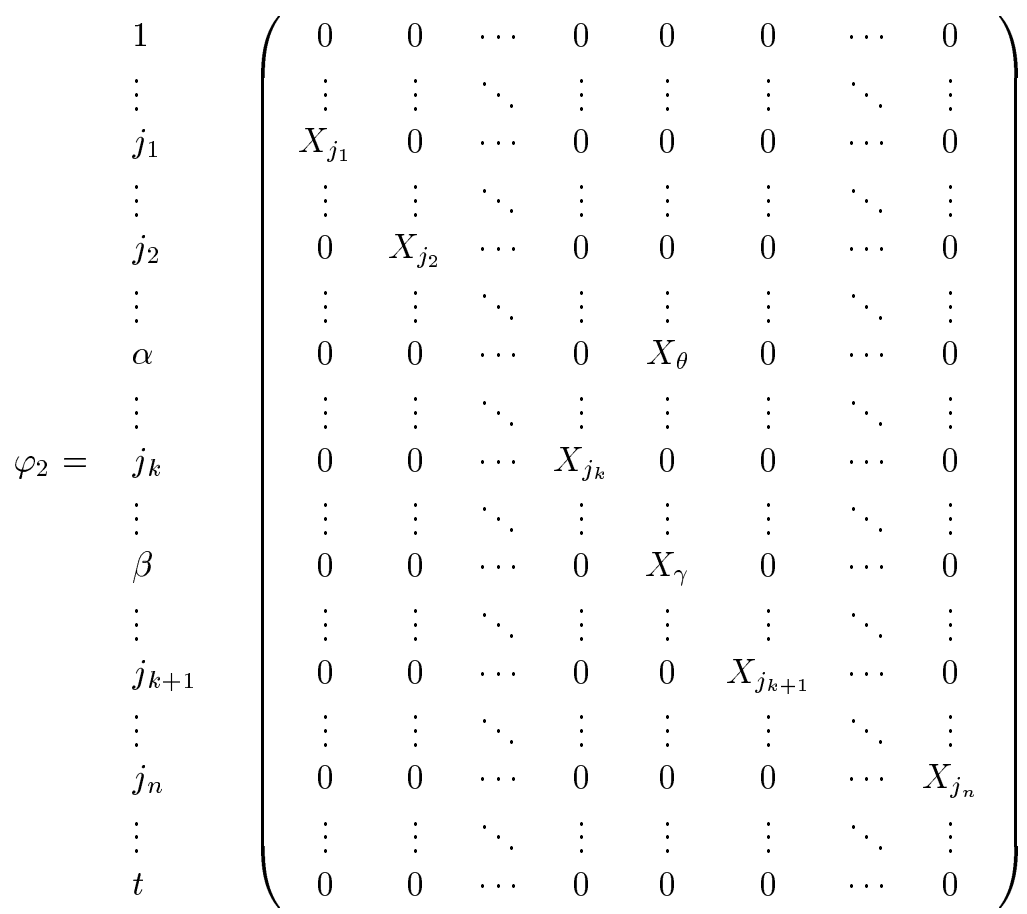

$$
\begin{aligned}
& \varphi_{3}=\left(\begin{array}{cccccc}
X_{j_{1}} & \cdots & 0 & 0 & \cdots & 0 \\
\vdots & \ddots & \vdots & \vdots & \ddots & \cdots \\
0 & \cdots & X_{j_{k}} & 0 & \cdots & 0 \\
0 & \cdots & 0 & 0 & \cdots & 0 \\
0 & \cdots & 0 & X_{j_{k+1}} & \cdots & 0 \\
\vdots & \ddots & \vdots & \vdots & \ddots & \cdots \\
0 & \cdots & 0 & 0 & \cdots & X_{j_{n}}
\end{array}\right) \\
& \varphi=\left(\begin{array}{cccc}
X_{j_{1}} & 0 & \cdots & 0 \\
0 & X_{j_{2}} & \cdots & 0 \\
\vdots & \vdots & \ddots & \cdots \\
0 & 0 & \cdots & X_{j_{n}}
\end{array}\right)
\end{aligned}
$$




\section{Diagram 4.1}

$$
\begin{aligned}
& \left.\begin{array}{l}
1 \\
\vdots \\
j_{1} \\
\vdots \\
j_{2} \\
\vdots \\
j_{i} \\
\vdots \\
j_{i+1} \\
\vdots \\
j_{n}
\end{array} \quad \begin{array}{cccccccc}
0 & 0 & \cdots & 0 & 0 & 0 & \cdots & 0 \\
\vdots & \vdots & \ddots & \vdots & \vdots & \vdots & \ddots & \vdots \\
X_{j_{1}} & 0 & \cdots & 0 & 0 & 0 & \cdots & 0 \\
\vdots & \vdots & \ddots & \vdots & \vdots & \vdots & \ddots & \vdots \\
\vdots & X_{j_{2}} & \cdots & 0 & 0 & 0 & \cdots & 0 \\
\vdots & \vdots & \ddots & \vdots & \vdots & \vdots & \ddots & \vdots \\
0 & 0 & \cdots & X_{j_{i}} & X_{j_{k}}+c X_{j_{l}} & 0 & \cdots & 0 \\
\vdots & \vdots & \ddots & \vdots & \vdots & \vdots & \ddots & \vdots \\
0 & 0 & \cdots & 0 & 0 & X_{j_{i+1}} & \cdots & 0 \\
\vdots & \vdots & \ddots & \vdots & \vdots & \vdots & \ddots & \vdots \\
0 & 0 & \cdots & 0 & 0 & 0 & \cdots & X_{j_{n}} \\
\vdots & \vdots & \ddots & \vdots & \vdots & \vdots & \ddots & \vdots \\
0 & 0 & \cdots & 0 & 0 & 0 & \cdots & 0
\end{array}\right) \\
& \varphi=\begin{array}{l}
1 \\
2 \\
\vdots \\
i+1 \\
i+2 \\
\vdots \\
n+1
\end{array} \quad\left(\begin{array}{cccccccc}
X_{j_{1}} & 0 & \cdots & 0 & 0 & 0 & \cdots & 0 \\
0 & X_{j_{2}} & \cdots & 0 & 0 & 0 & \cdots & 0 \\
\vdots & \vdots & \ddots & \vdots & \vdots & \vdots & \ddots & \vdots \\
0 & 0 & \cdots & X_{j_{i}} & X_{j_{k}}+c X_{j_{l}} & 0 & \cdots & 0 \\
0 & 0 & \cdots & 0 & 0 & 0 & \cdots & 0 \\
0 & 0 & \cdots & 0 & 0 & X_{j_{i+1}} & \cdots & 0 \\
\vdots & \vdots & \ddots & \vdots & \vdots & \vdots & \ddots & \vdots \\
0 & 0 & \cdots & 0 & 0 & 0 & \cdots & X_{j_{n}}
\end{array}\right)
\end{aligned}
$$




\section{Diagram 4.2}

$$
\begin{aligned}
& \left.\begin{array}{c}
1 \\
\vdots \\
j_{1} \\
\vdots \\
j_{2} \\
\vdots \\
j_{l} \\
j_{l}
\end{array} \begin{array}{cccccccccccc}
0 & 0 & \cdots & 0 & \cdots & 0 & \cdots & 0 & \cdots & 0 & 0 \\
\vdots & \vdots & \ddots & \vdots & \ddots & \vdots & \ddots & \vdots & \ddots & \vdots & \vdots \\
X_{j_{1}} & 0 & \cdots & 0 & \cdots & 0 & \cdots & 0 & \cdots & 0 & 0 \\
\vdots & \vdots & \ddots & \vdots & \ddots & \vdots & \ddots & \ddots & \vdots & \vdots & \\
j_{i} & \vdots & X_{j_{2}} & \cdots & 0 & \cdots & 0 & \cdots & 0 & \cdots & 0 & 0 \\
\vdots & \vdots & \ddots & \vdots & \ddots & \vdots & \ddots & \vdots & \ddots & \vdots & \vdots \\
j_{k} & \vdots & \vdots & \ddots & X_{j_{l}} & \cdots & 0 & \cdots & 0 & \cdots & 0 & c X_{j_{i}} \\
\vdots & 0 & 0 & \cdots & 0 & \cdots & \vdots & \ddots & \vdots & \ddots & \vdots & \vdots \\
j_{n} & \vdots & \vdots & \ddots & \vdots & \ddots & \vdots & \ddots & \vdots & \ddots & \vdots & \vdots \\
\vdots & 0 & 0 & \cdots & 0 & \cdots & 0 & \cdots & X_{j_{k}} & \cdots & 0 & X_{j_{i}} \\
t & \vdots & \vdots & \ddots & \vdots & \ddots & \vdots & \ddots & \vdots & \ddots & \vdots & \vdots \\
0 & 0 & \cdots & 0 & \cdots & 0 & \cdots & 0 & \cdots & X_{j_{n}} & 0 \\
\vdots & \vdots & \ddots & \vdots & \ddots & \vdots & \ddots & \vdots & \ddots & \vdots & \vdots \\
0 & 0 & \cdots & 0 & \cdots & 0 & \cdots & 0 & \cdots & 0 & 0
\end{array}\right) \\
& \varphi=\left(\begin{array}{cccccc}
X_{j_{1}} & 0 & 0 & \cdots & 0 & 0 \\
0 & X_{j_{2}} & 0 & \cdots & 0 & 0 \\
0 & 0 & X_{j_{3}} & \cdots & 0 & 0 \\
\vdots & \vdots & \vdots & \ddots & \vdots & \vdots \\
0 & 0 & 0 & \cdots & X_{j_{n}} & 0 \\
0 & 0 & 0 & \cdots & 0 & X_{j_{i}}
\end{array}\right)
\end{aligned}
$$




\section{Diagram 4.3}

$$
\left.\begin{array}{l|cccccccccccc}
1 & 0 & 0 & \cdots & 0 & 0 & 0 & \cdots & 0 & 0 & 0 & \cdots & 0 \\
\vdots & \vdots & \vdots & \ddots & \vdots & \vdots & \vdots & \ddots & \vdots & \vdots & \vdots & \ddots & \vdots \\
k_{s_{1}}^{1} & 0 & 0 & \cdots & 0 & c_{s_{1}}^{1} X_{k_{s_{1}}^{1}} & 0 & \cdots & 0 & 0 & 0 & \cdots & 0 \\
\vdots & \vdots & \vdots & \ddots & \vdots & \vdots & \vdots & \ddots & \vdots & \vdots & \vdots & \ddots & \vdots \\
j_{1} & X_{j_{1}} & 0 & \cdots & 0 & 0 & 0 & \cdots & 0 & 0 & 0 & \cdots & 0 \\
\vdots & \vdots & \ddots & \vdots & \vdots & \vdots & \ddots & \vdots & \vdots & \vdots & \ddots & \vdots \\
k_{2}^{1} & 0 & 0 & \cdots & 0 & c_{2}^{1} X_{k_{2}^{1}} & 0 & \cdots & 0 & 0 & 0 & \cdots & 0 \\
\vdots & \vdots & \vdots & \ddots & \vdots & \vdots & \vdots & \ddots & \vdots & \vdots & \vdots & \ddots & \vdots \\
j_{2} & 0 & X_{j_{2}} & \cdots & 0 & 0 & 0 & \cdots & 0 & 0 & 0 & \cdots & 0 \\
\vdots & \vdots & \vdots & \ddots & \vdots & \vdots & \vdots & \ddots & \vdots & \vdots & \vdots & \ddots & \vdots \\
k_{s_{z}}^{z} & 0 & 0 & \cdots & 0 & 0 & 0 & \cdots & 0 & c_{s_{z}}^{z} X_{k_{s_{z}}^{z}} & 0 & \cdots & 0 \\
\vdots & \vdots & \vdots & \ddots & \vdots & \vdots & \vdots & \ddots & \vdots & \vdots & \vdots & \ddots & \vdots \\
j_{c_{1}} & 0 & 0 & \cdots & X_{j_{1}} & 0 & 0 & \cdots & 0 & 0 & 0 & \cdots & 0 \\
\vdots & \vdots & \vdots & \ddots & \vdots & \vdots & \vdots & \ddots & \vdots & \vdots & \vdots & \ddots & \vdots \\
k_{1}^{1} & 0 & 0 & \cdots & 0 & X_{k_{1}^{1}} & 0 & \cdots & 0 & 0 & 0 & \cdots & 0 \\
\vdots & \vdots & \vdots & \ddots & \vdots & \vdots & \vdots & \ddots & \vdots & \vdots & \vdots & \ddots & \vdots \\
j_{c_{1}+1} & 0 & 0 & \cdots & 0 & 0 & X_{c_{1}+1} & \cdots & 0 & 0 & 0 & \cdots & 0 \\
\vdots & \vdots & \vdots & \ddots & \vdots & \vdots & \vdots & \ddots & \vdots & \vdots & \vdots & \ddots & \vdots \\
j_{c_{z}} & 0 & 0 & \cdots & 0 & 0 & 0 & \cdots & X_{j_{z}} & 0 & 0 & \cdots & 0 \\
\vdots & \vdots & \vdots & \ddots & \vdots & \vdots & \vdots & \ddots & \vdots & \vdots & \vdots & \ddots & \vdots \\
k_{z}^{1} & \vdots & 0 & \cdots & 0 & 0 & 0 & \cdots & 0 & X_{k_{z}^{1}} & 0 & \cdots & 0 \\
\vdots & \vdots & \vdots & \ddots & \vdots & \vdots & \vdots & \ddots & \vdots & \vdots & \vdots & \ddots & \vdots \\
j_{c_{z}+1} & 0 & 0 & \cdots & 0 & 0 & 0 & \cdots & 0 & 0 & X_{j_{c_{z}+1}} & \cdots & 0 \\
\vdots & \vdots & \vdots & \ddots & \vdots & \vdots & \vdots & \ddots & \vdots & \vdots & \vdots & \ddots & \vdots \\
j_{n} & 0 & 0 & \cdots & 0 & 0 & 0 & \cdots & 0 & 0 & \cdots & X_{j_{n}} & \\
\vdots & \vdots & \vdots & \ddots & \vdots & \vdots & \vdots & \ddots & \vdots & \vdots & \vdots & \ddots & \vdots \\
0 & 0 & \cdots & 0 & 0 & 0 & \cdots & 0 & 0 & 0 & \cdots & 0
\end{array}\right)
$$


Diagram 4.3, cont.

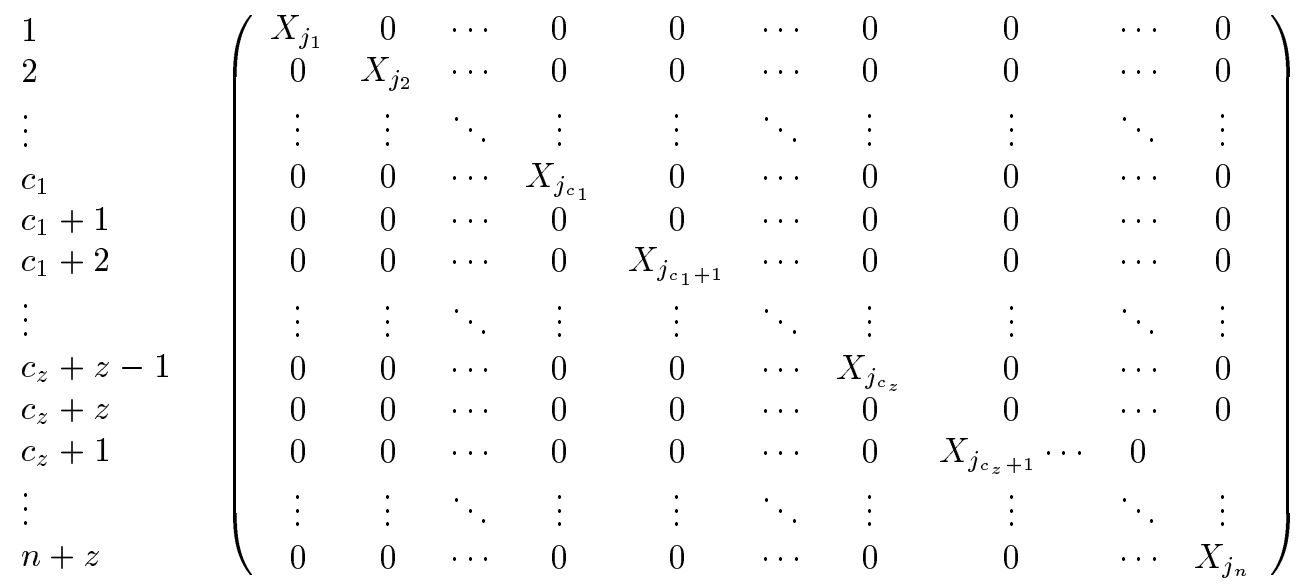

$$
\begin{aligned}
& \varphi=\left(\begin{array}{ccccc}
X_{j_{1}} & 0 & 0 & \cdots & 0 \\
0 & X_{j_{2}} & 0 & \cdots & 0 \\
0 & 0 & X_{j_{3}} & \cdots & 0 \\
\vdots & \vdots & \vdots & \ddots & \vdots \\
0 & 0 & 0 & \cdots & X_{j_{n}}
\end{array}\right)
\end{aligned}
$$




\section{Diagram 5.1}

CASE I: Suppose $\operatorname{tip}\left(X_{j_{i}} X_{\beta}+c X_{\xi} X_{j_{i}}\right)=X_{j_{i}} X_{\beta}$.

$\underline{\text { Case } 1}$ Suppose $X_{\xi}=X_{j_{k}}$ for some $k, X_{\beta} \neq X_{j_{l}}$ for all $l$.

$$
\begin{aligned}
& \left.\begin{array}{l|cccccccccc}
1 & 0 & \cdots & 0 & 0 & \cdots & 0 & 0 & 0 & \cdots & 0 \\
\vdots & \ddots & \vdots & \vdots & \ddots & \vdots & \vdots & \vdots & \ddots & \vdots \\
j_{1} & \vdots & & & & \\
X_{j_{1}} & \cdots & 0 & 0 & \cdots & 0 & 0 & 0 & \cdots & 0 \\
\vdots & \ddots & \vdots & \vdots & \ddots & \vdots & \vdots & \vdots & \ddots & \vdots \\
\vdots & 0 & \cdots & X_{j_{k}} & 0 & \cdots & 0 & c X_{j_{i}} & 0 & \cdots & 0 \\
j_{k+1} & \vdots & \ddots & \vdots & \vdots & \ddots & \vdots & \vdots & \vdots & \ddots & \vdots \\
\vdots & 0 & \cdots & 0 & X_{j_{k+1}} & \cdots & 0 & 0 & 0 & \cdots & 0 \\
j_{i} & \vdots & \ddots & \vdots & \vdots & \ddots & \vdots & \vdots & \vdots & \ddots & \vdots \\
\vdots & 0 & \cdots & 0 & 0 & \cdots & X_{j_{i}} & X_{\beta} & 0 & \cdots & 0 \\
j_{i+1} & \vdots & \ddots & \vdots & \vdots & \ddots & \vdots & \vdots & \vdots & \ddots & \vdots \\
\vdots & \vdots & \cdots & 0 & 0 & \cdots & 0 & 0 & X_{j_{i+1}} & \cdots & 0 \\
j_{n} & \vdots & \ddots & \vdots & \vdots & \ddots & \vdots & \vdots & \vdots & \ddots & \vdots \\
\vdots & \cdots & 0 & 0 & \cdots & 0 & 0 & 0 & \cdots & X_{j_{n}} \\
\vdots & \vdots & \ddots & \vdots & \vdots & \ddots & \vdots & \vdots & \vdots & \ddots & \vdots \\
0 & \cdots & 0 & 0 & \cdots & 0 & 0 & 0 & \cdots & 0
\end{array}\right) \\
& \varphi_{3}=\begin{array}{l}
1 \\
2 \\
\vdots \\
i \\
i+1 \\
i+2 \\
\vdots \\
n+1
\end{array} \quad\left(\begin{array}{ccccccccc}
X_{j_{1}} & 0 & \cdots & 0 & 0 & 0 & 0 & \cdots & 0 \\
0 & X_{j_{2}} & \cdots & 0 & 0 & 0 & 0 & \cdots & 0 \\
\vdots & \vdots & \ddots & \vdots & \vdots & \vdots & \vdots & \ddots & \vdots \\
0 & 0 & \cdots & X_{j_{i}} & X_{\beta} X_{j_{i}} & X_{j_{i}}^{2} & 0 & \cdots & 0 \\
0 & 0 & \cdots & 0 & 0 & 0 & 0 & \cdots & 0 \\
0 & 0 & \cdots & 0 & 0 & 0 & X_{j_{i+1}} & \cdots & 0 \\
\vdots & \vdots & \ddots & \vdots & \vdots & \vdots & \vdots & \ddots & \vdots \\
0 & 0 & \cdots & 0 & 0 & 0 & 0 & \cdots & X_{j_{n}}
\end{array}\right)
\end{aligned}
$$


Diagram 5.1, cont.

$\underline{\text { Case } 2}$ Suppose $X_{\beta}=X_{j_{l}}$ for some $l, X_{\xi} \neq X_{j_{k}}$ for all $k$.

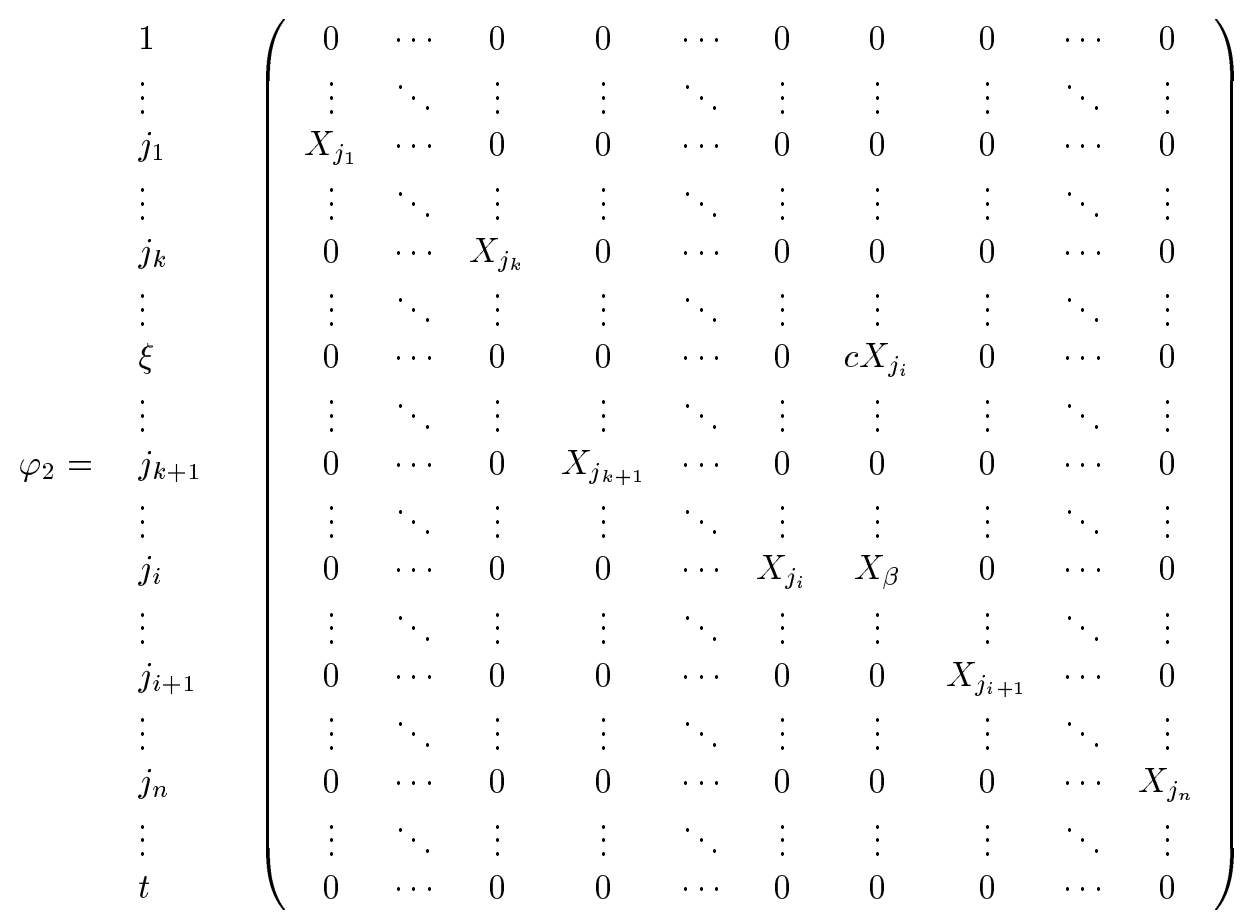

\begin{tabular}{|c|c|c|c|c|c|c|c|c|c|c|c|c|c|}
\hline \multicolumn{14}{|c|}{$\varphi_{3}=$} \\
\hline 1 & $X_{j_{1}}$ & 0 & $\ldots$ & 0 & 0 & 0 & $\ldots$ & 0 & 0 & 0 & 0 & $\ldots$ & 0 \\
\hline 2 & 0 & $X_{j_{2}}$ & $\cdots$ & 0 & 0 & 0 & $\cdots$ & 0 & 0 & 0 & 0 & $\cdots$ & 0 \\
\hline : & $\vdots$ & $\vdots$ & $\ddots$. & $\vdots$ & $\vdots$ & $\vdots$ & $\ddots$ & $\vdots$ & $\vdots$ & $\vdots$ & $\vdots$ & $\ddots$ & $\vdots$ \\
\hline$k$ & 0 & 0 & $\ldots$ & $X_{j_{k}}$ & 0 & 0 & $\ldots$ & 0 & 0 & 0 & 0 & $\ldots$ & 0 \\
\hline$k+1$ & 0 & 0 & $\cdots$ & 0 & 0 & $X_{j_{k+1}}$ & $\cdots$ & 0 & 0 & 0 & 0 & $\cdots$ & 0 \\
\hline$\vdots$ & $\vdots$ & $\vdots$ & $\ddots$ & $\vdots$ & $\vdots$ & $\vdots$ & $\ddots$ & $\vdots$ & $\vdots$ & $\vdots$ & $\vdots$ & $\ddots$ & $\vdots$ \\
\hline$i-1$ & 0 & 0 & $\cdots$ & 0 & 0 & 0 & $\cdots$ & $X_{j_{i-1}}$ & 0 & 0 & 0 & $\ldots$ & 0 \\
\hline$i$ & 0 & 0 & $\cdots$ & 0 & 0 & 0 & $\ldots$ & 0 & $X_{j_{i}}$ & $X_{\beta} X_{j_{i}}$ & 0 & $\ldots$ & 0 \\
\hline$i+1$ & 0 & 0 & $\cdots$ & 0 & $X_{\beta} X_{j_{i}}$ & 0 & $\cdots$ & 0 & 0 & 0 & 0 & $\ldots$ & 0 \\
\hline$i+2$ & 0 & 0 & $\cdots$ & 0 & 0 & 0 & $\cdots$ & 0 & 0 & 0 & $X_{j_{i+1}}$ & $\cdots$ & 0 \\
\hline$\vdots$ & $\vdots$ & $\vdots$ & $\ddots$ & $\vdots$ & $\vdots$ & $\vdots$ & $\ddots$ & $\vdots$ & $\vdots$ & $\vdots$ & $\vdots$ & $\ddots$ & $\vdots$ \\
\hline$n+1$ & 0 & 0 & $\cdots$ & 0 & 0 & 0 & $\ldots$ & 0 & 0 & 0 & 0 & $\ldots$ & $X_{j_{n}}$ \\
\hline
\end{tabular}


Diagram 5.1, cont.

$\underline{\text { Case } 3}$ Suppose $X_{\beta}=X_{j_{l}}$ for some $l, X_{\xi}=X_{j_{k}}$ for some $k$.

$\varphi_{2}$ is the same as in Case 1.

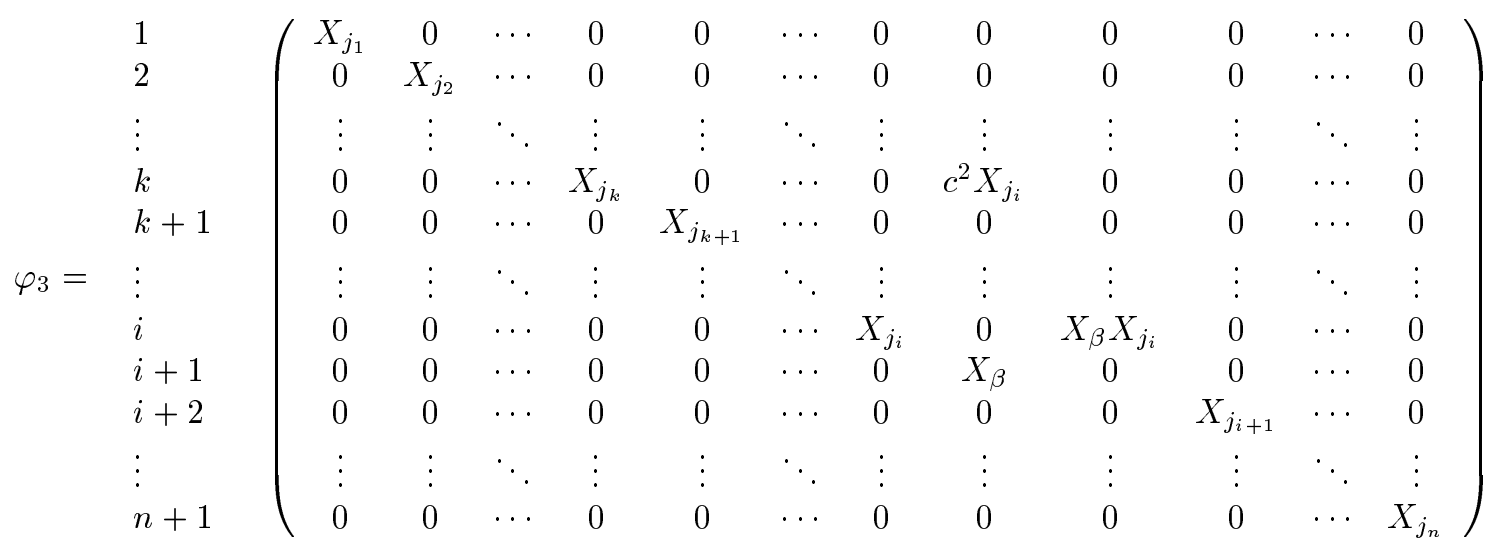


Diagram 5.1, cont.

CASE II: Suppose $\operatorname{tip}\left(X_{j_{i}} X_{\beta}+c X_{\xi} X_{j_{i}}\right)=X_{\xi} X_{j_{i}}$.

Case 1 Suppose $X_{\xi}=X_{j_{k}}$ for some $k, X_{\beta} \neq X_{j_{l}}$ for all $l$.

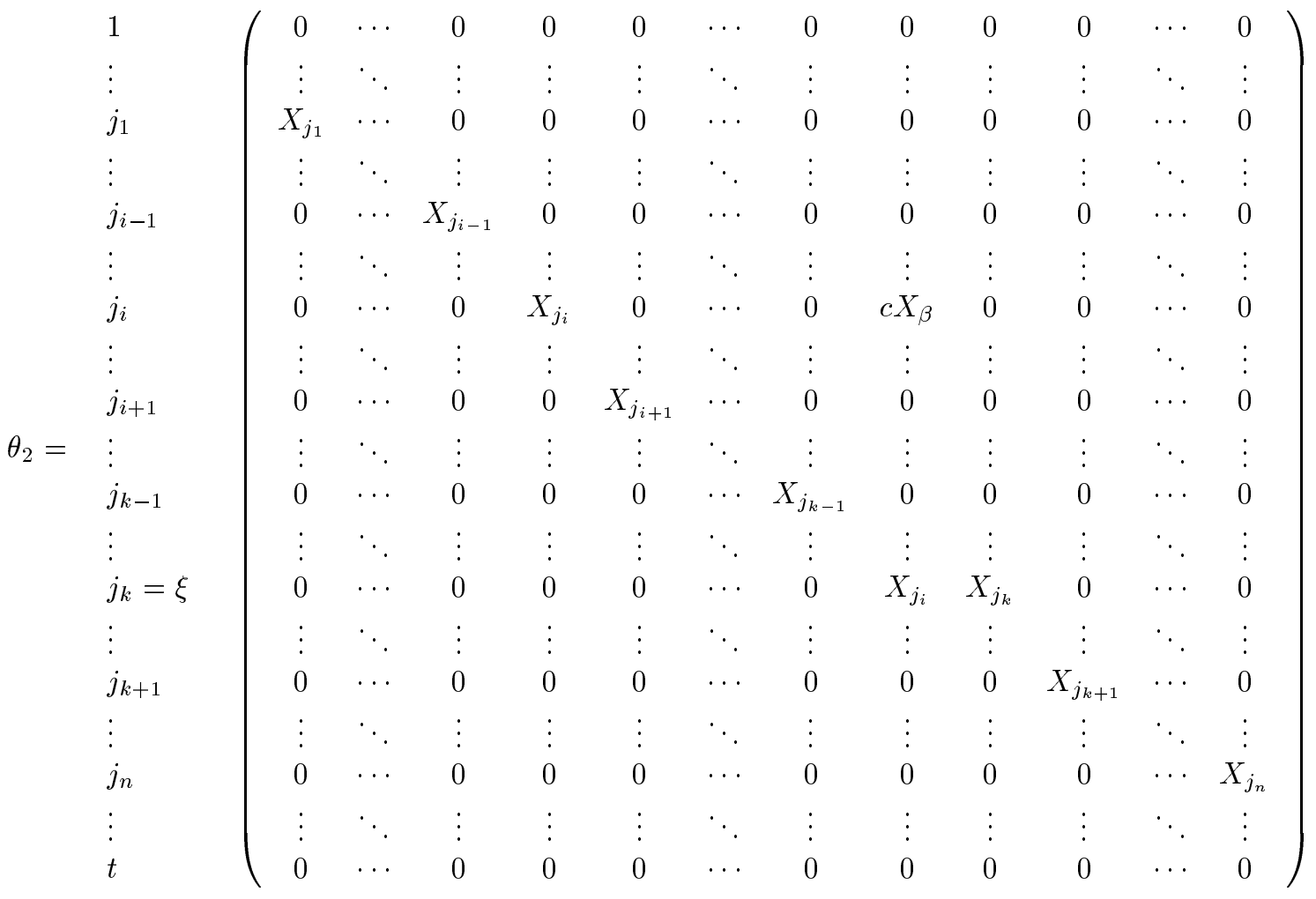

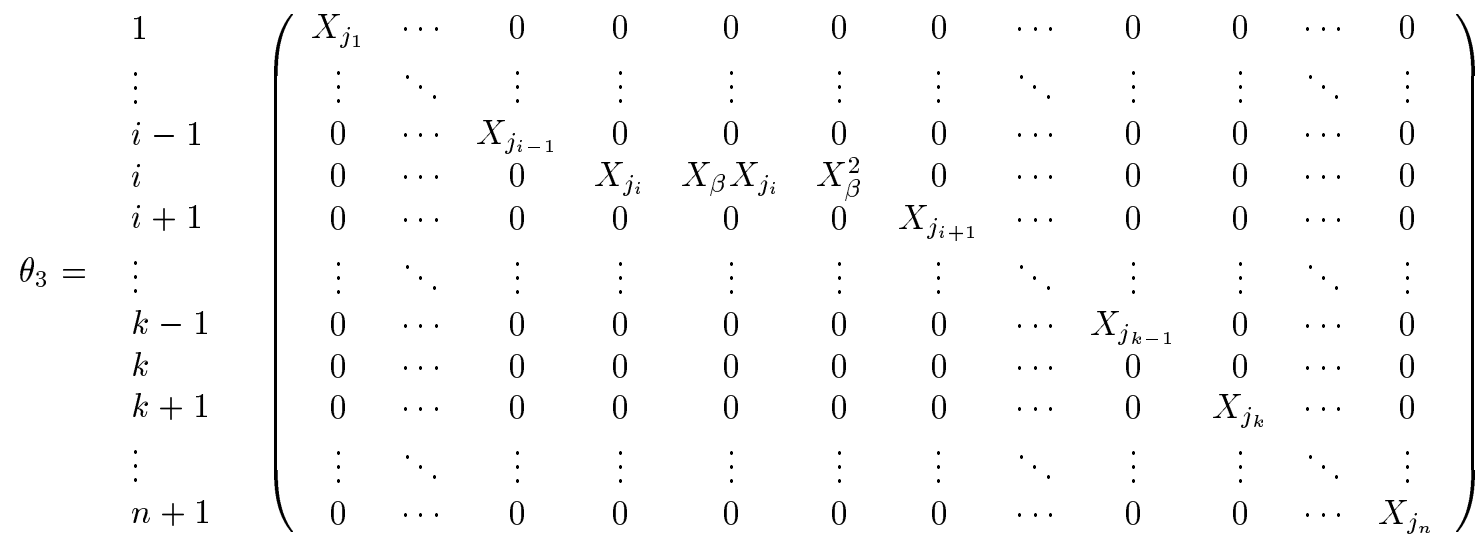


Diagram 5.1, cont.

$\underline{\text { Case } 2}$ Suppose $X_{\beta}=X_{j_{l}}$ for some $l, X_{\xi} \neq X_{j_{k}}$ for all $k$.

$$
\begin{aligned}
& \left.\begin{array}{l|ccccccccccc}
1 \\
\vdots \\
j_{1} \\
\vdots \\
j_{i-1} \\
\vdots \\
j_{i} \\
\vdots \\
j_{i+1} \\
\vdots & \vdots & \ddots & \vdots & \vdots & \vdots & \ddots & \vdots & \vdots & \vdots & \ddots & \vdots \\
X_{j_{1}} & \cdots & 0 & 0 & 0 & \cdots & 0 & 0 & 0 & \cdots & 0 \\
\vdots & \ddots & \vdots & \vdots & \vdots & \ddots & \vdots & \vdots & \vdots & \ddots & \vdots \\
j_{k} & \cdots & X_{j_{i-1}} & 0 & 0 & \cdots & 0 & 0 & 0 & \cdots & 0 \\
\vdots & \vdots & \ddots & \vdots & \vdots & \vdots & \ddots & \vdots & \vdots & \vdots & \ddots & \vdots \\
\xi & \cdots & 0 & X_{j_{i}} & 0 & \cdots & 0 & c X_{\beta} & 0 & \cdots & 0 \\
\vdots & \ddots & \vdots & \vdots & \vdots & \ddots & \vdots & \vdots & \vdots & \ddots & \vdots \\
\vdots & \vdots & \cdots & 0 & 0 & X_{j_{i+1}} & \cdots & 0 & 0 & 0 & \cdots & 0 \\
j_{k+1} & \vdots & \ddots & \vdots & \vdots & \vdots & \ddots & \vdots & \vdots & \vdots & \ddots & \vdots \\
\vdots & 0 & \cdots & 0 & 0 & 0 & \cdots & X_{j_{k}} & 0 & 0 & \cdots & 0 \\
j_{n} & \vdots & \ddots & \vdots & \vdots & \vdots & \ddots & \vdots & \vdots & \vdots & \ddots & \vdots \\
\vdots & 0 & \cdots & 0 & 0 & 0 & \cdots & 0 & X_{j_{i}} & 0 & \cdots & 0 \\
t & \vdots & \ddots & \vdots & \vdots & \vdots & \ddots & \vdots & \vdots & \vdots & \ddots & \vdots \\
0 & \cdots & 0 & 0 & 0 & \cdots & 0 & 0 & X_{j_{k+1}} & \cdots & 0 \\
\vdots & \ddots & \vdots & \vdots & \vdots & \ddots & \vdots & \vdots & \vdots & \ddots & \vdots \\
0 & \cdots & 0 & 0 & 0 & \cdots & 0 & 0 & 0 & \cdots & X_{j_{n}} \\
\vdots & \ddots & \vdots & \vdots & \vdots & \ddots & \vdots & \vdots & \vdots & \ddots & \vdots \\
0 & \cdots & 0 & 0 & 0 & \cdots & 0 & 0 & 0 & \cdots & 0
\end{array}\right)
\end{aligned}
$$

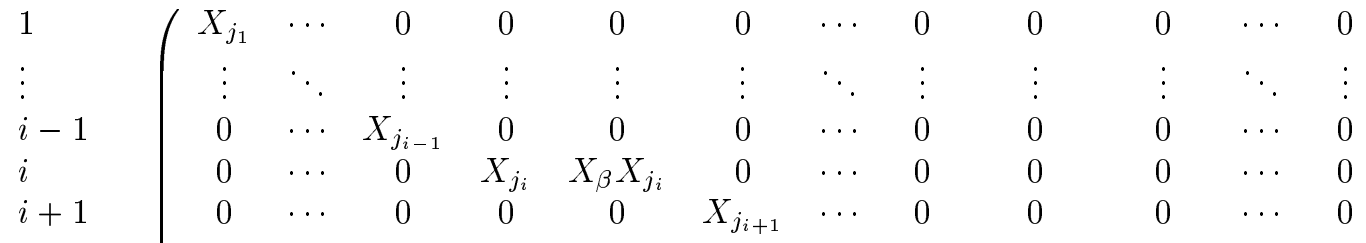

$$
\begin{aligned}
& \begin{array}{l|llllllllllll}
\theta_{3}= & \vdots & \vdots & \ddots & \vdots & \vdots & \vdots & \vdots & \ddots & \vdots & \vdots & \vdots & \ddots \\
\hline
\end{array} \\
& \begin{array}{lllllllllllll}
k & 0 & \cdots & 0 & 0 & 0 & 0 & \cdots & X_{j_{k}} & 0 & 0 & \cdots & 0 \\
0 & \cdots & 0 & 0 & 0 & 0 & \cdots & 0 & X_{0} X_{j} & 0 & \cdots & 0
\end{array} \\
& \begin{array}{c|cccccccccccc}
k+1 & 0 & \cdots & 0 & 0 & 0 & 0 & \cdots & 0 & X_{\beta} X_{j_{i}} & 0 & \cdots & 0 \\
k+2 & 0 & \cdots & 0 & 0 & 0 & 0 & \cdots & 0 & 0 & X_{j_{k+1}} & \cdots & 0 \\
\vdots & \vdots & \ddots & \vdots & \vdots & \vdots & \vdots & \ddots & \vdots & \vdots & \vdots & \ddots & \vdots
\end{array}
\end{aligned}
$$

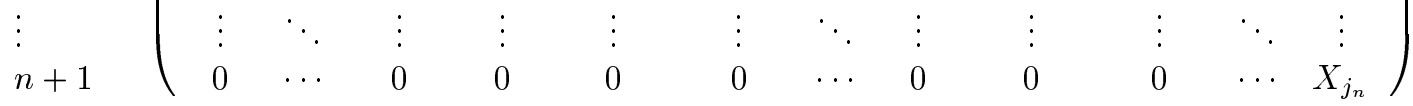




\section{Diagram 5.1, cont.}

$\underline{\text { Case } 3}$ Suppose $X_{\beta}=X_{j_{l}}$ for some $l, X_{\xi}=X_{j_{k}}$ for some $k$.

$\theta_{2}$ is the same as in Case 1.

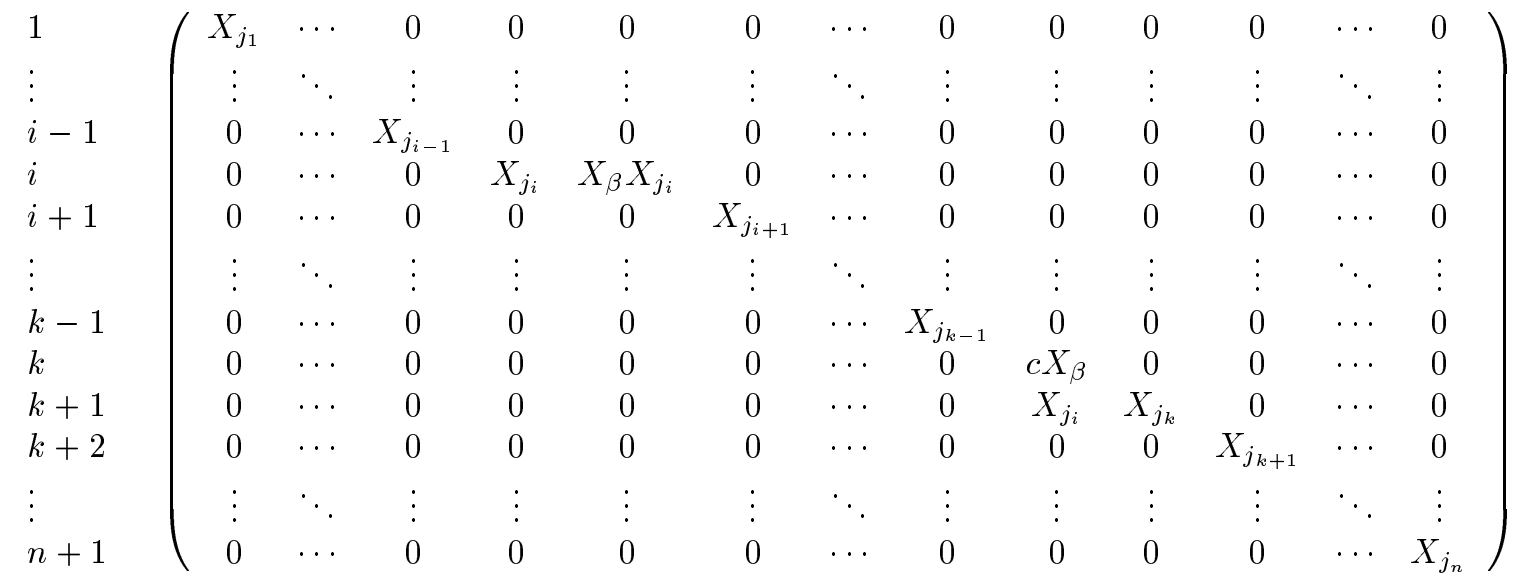




\section{Bibliography}

[1] J. Backelin and R. Fröberg Koszul Algebras, Veronese Subrings and Rings with Linear Resolutions Rev. Roumaine Math. Pures Appl. 30 (1985) 85-97

[2] A.I. Bondal Helices, Representations of Quivers and Koszul Algebras. London Math. Soc. Lecture Notes Ser. 148 (1990) 75-95

[3] W. Bruns, J. Gubeladze and Nga Viet Trung Normal Polytopes, triagulations, and Koszul Algebras J. Reine Angew. Math. 485 (1997) 123-160

[4] E.Cline, B.Parshall, and L. Scott Finite Dimensional Algebras and Highest weight Categories J. Reine Angew. Math. 391 (1988) 85-99

[5] D. Cox, J. Little, and D. O'Shea Ideals, Varieties, and Algorithms Springer-Verlag (1992)

[6] Edward L. Green Introduction to Koszul Algebras Representation theory and algebraic geometry (Waltham, MA, 1995) London Math. Soc. Lecture Note Ser., 238, Cambridge Univ. Press, Cambridge (1997)

[7] Edward L. Green Introduction to Noncommutative Gröbner Bases (1995)

[8] Edward L. Green and Roberto Martinez-Villa Koszul and Yoneda Algebras Canad. Math. Soc., Conference Proceedings 18 Representation Theory of Algebras (1994) 247298

[9] Edward L. Green and Rosa Huang Projective Resolutions of Straightening Closed Algebras Generated by Minors Adv. in Math. 110 (1995) 314-333

[10] Edward L. Green and D. Zacharia The Cohomology ring of a monomial algebra Manuscripta Math. 85 (1994)

[11] Yu Manin Some Remarks on Koszul Algebras and Quantum Groups Ann. Inst. Fourier, Grenoble 37 (1987) 4 191-205

[12] S.B.Priddy Koszul Resolutions Trans A.M.S. 152 (1970) 39-60 


\section{Heather C. McGilvray \\ 2007 63rd St. SE \\ Everett, WA 98203 \\ (425)353-6462 \\ mcgilvra@math.vt.edu}

\section{Education}

July 1998

1995

1992

Ph.D.(Computational Algebra)

Virginia Polytechnic Institute and State University

Blacksburg, VA

Master of Science (Mathematics)

Virginia Polytechnic Institute and State University

Blacksburg, VA

Bachelor of Science(Mathematics)

Texas A\&M University

College Station, TX

Experience

$07 / 93-05 / 97$

$09 / 92-06 / 93$

$09 / 90-05 / 92$

\section{Computer Skills}

- Familar with VAX Macintosh, and Windows operating systems

- Knowledgeable in programming: $\mathrm{C}, \mathrm{C}++$, and Fortran

- Experience with Mathematica, Groebner, LaTEX

Accomplishments and Awards

- High teaching evaluations from both mentors and students

- Texas A\&M University Senior Honors Thesis :

On Dependence and Independence in Near -Rings (1992)

- National Merit Scholar (1998) 